THE ROLE OF SYSTEM DYNAMICS ON THE BEHAVIOR OF ELASTOMERIC FRICTION

by

Robert S. De Togni

Thesis submitted to the Faculty of the

Virginia Polytechnic Institute and State University

in partial fulfillment of the requirements for the degree of

MASTER OF SCIENCE

in

Mechanical Engineering

APPROVED:
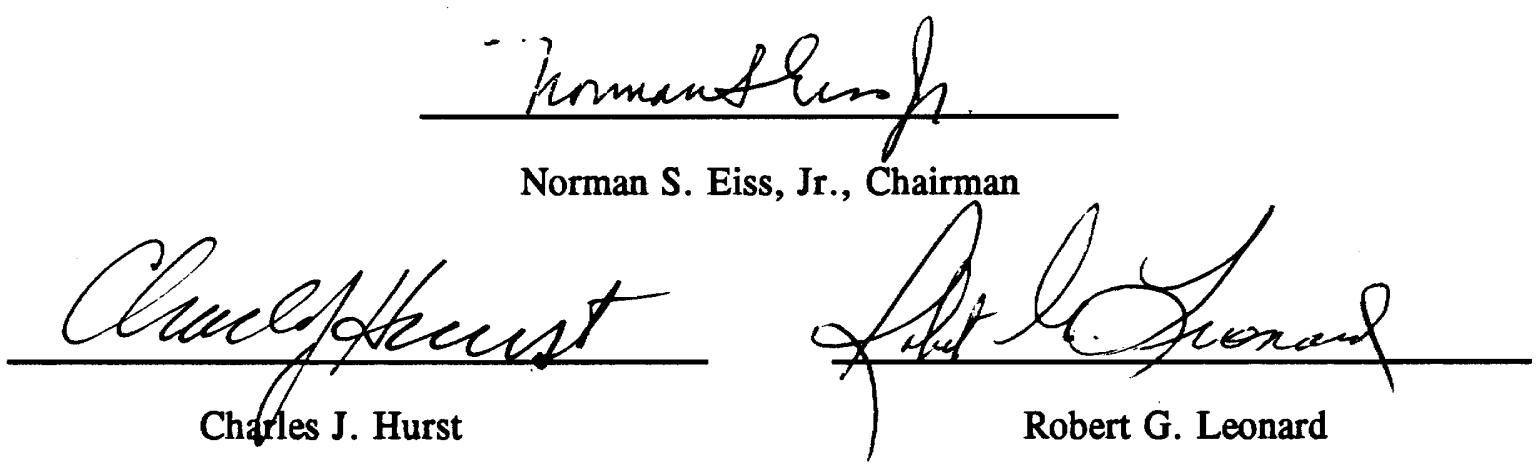

May 1992

Blacksburg, Virginia 


$\begin{array}{cc} & 20 \\ & 5605 \\ 0.2 & 1859 \\ & 1992 \\ & 0486 \\ & 0.2\end{array}$




\title{
THE ROLE OF SYSTEM DYNAMICS ON THE BEHAVIOR OF ELASTOMERIC FRICTION
}

\author{
by \\ Robert S. De Togni \\ Norman S. Eiss, Jr., Chairman \\ Mechanical Engineering \\ (ABSTRACT)
}

Friction induced vibration of an epoxy coated shaft rotating in an elastomeric bushing is investigated. This study investigates the manner in which system dynamics and friction mechanisms are responsible for friction induced vibration and noise generation. A test method was developed to measure the friction torque and the system and acoustic response of the sliding system. Several materials including a fluorocarbon elastomer, a polydimethylsiloxane, and a natural rubber were tested.

Three friction regimes were observed which were stick-slip oscillations, quasi-harmonic oscillations, and steady sliding. System stiffness and load were varied to observe changes in the critical velocities bounding each regime. System parameters were varied to determine sliding conditions leading to self-induced vibration, to establish how the character of vibration is affected, and to correlate friction torque with system and acoustic vibration for each elastomeric material.

A two degree-of-freedom, lumped parameter model was developed to simulate the effect of system dynamics on the sliding behavior of the elastomeric bushing. The comparison of simulated and experimental response using analyses in the time and frequency domain indicate the predictive model provides an excellent representation of stick-slip behavior at various operating conditions. 


\section{Acknowledgements}

I would like to express deepest thanks to Norman Eiss for his time, patience, and expertise that he gave me while completing this research. His openmindedness and insight allowed me to realize my research goals. I would also like to thank my advisors, Drs. Robert G. Leonard and Charles J. Hurst, for the research guidance in their areas of expertise.

I am thankful to Ford Motor Company for sponsoring this project and to the Gates Rubber Company for supplying additional test specimens. I express my appreciation to Paul Kilgoar and Robert Pett of Ford and John Connell at Gates for their support of this project. I also must thank Dr. Robert A. Comparin for providing additional stipends so that I could complete my studies and research.

There are enumerable persons to whom I owe a great deal for their selfless help and guidance. These include Drs. Mitchell, Fuller, and Robertshaw for use of their laboratory facilities and equipment and those persons working in the Instrumentation and Mechanical shops. Also I must include thanks to my colleagues and friends Ronald Rorrer, Brian Weick, Hamid Ghasemi, Bhawani Tripathy, Brian McCann, Thomas Warrington, and Joseph Liberti for our discussions and deliberations regarding this research and life itself. Special thanks must be expressed to all my friends with whom the time I spent made life enjoyable and complete.

Last and foremost I would like to thank God for my dear parents, Gino and Dorothy De Togni, and the rest of my family for their love and encouragement which I will never forget. 


\title{
Table of Contents
}

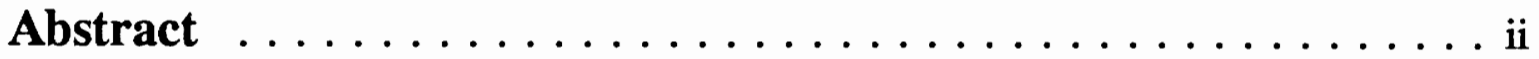

Acknowledgements $\ldots \ldots \ldots \ldots \ldots \ldots \ldots \ldots$ iii

Table of Contents $\ldots \ldots \ldots \ldots \ldots \ldots \ldots \ldots$ iv

List of Figures $\ldots \ldots \ldots \ldots \ldots \ldots \ldots$ viii

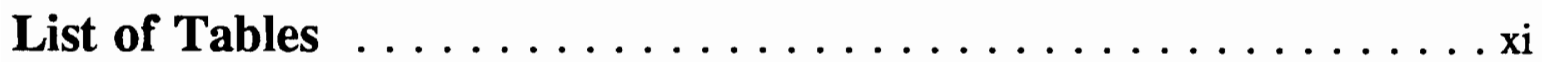

1.0 Introduction $\ldots \ldots \ldots \ldots \ldots \ldots \ldots \ldots \ldots \ldots \ldots$

2.0 Literature Review . . . . . . . . . . . . . . . 4

2.1 Friction Theories for Elastomers $\ldots \ldots \ldots \ldots \ldots \ldots$

2.2 Friction Induced Vibration $\ldots \ldots \ldots \ldots \ldots \ldots \ldots \ldots \ldots \ldots$

2.3 Role of System Dynamics . . . . . . . . . . . . 8

2.4 Acoustics and Friction Induced Vibration $\ldots \ldots \ldots \ldots \ldots \ldots$

Relationship to System Natural Frequencies

Relationship of Noise and Load, Velocity, Mass, Temperature, and Surface roughness

Relationship to Material

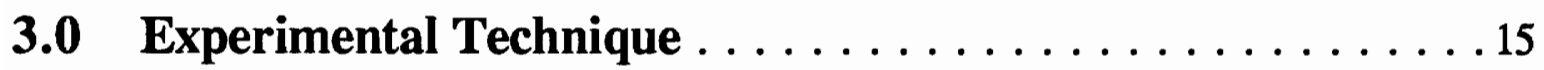

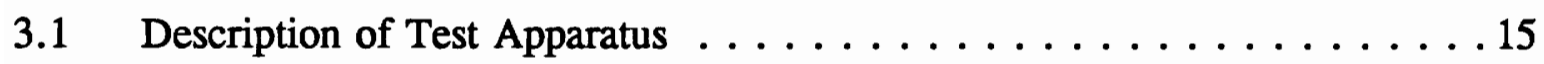

3.1.1 Configuration . . . . . . . . . . . . . . 15

3.1.2 Instrumentation and Data Acquisition $\ldots \ldots \ldots \ldots \ldots$

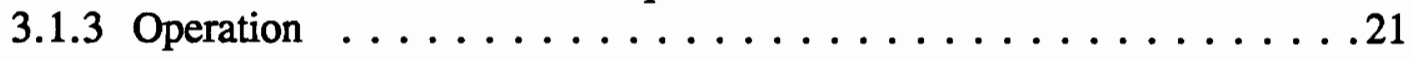




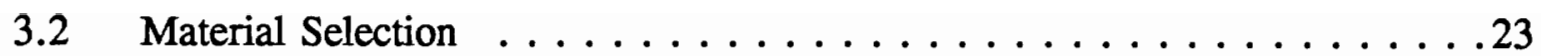

3.2.1 Fluorocarbon Elastomer . . . . . . . . . . . . . . 23

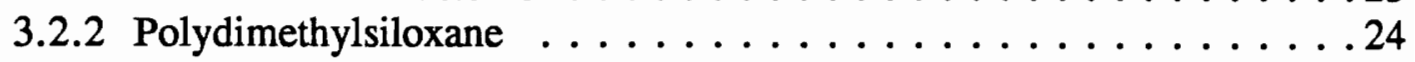

3.2.3 Natural Rubber . . . . . . . . . . . . . . . . . 25

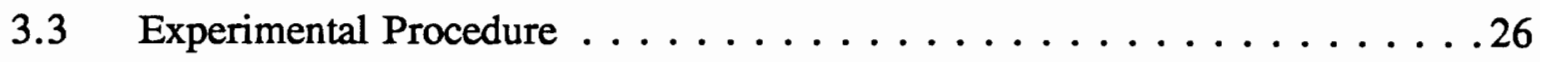

3.3.1 Friction/Velocity Study . . . . . . . . . . . . . 27
Regimes of Frictional Behavior
Effect of System Stiffness on $V_{\text {crit }}$
Effect of Normal Pressure
Summary of Test Conditions

3.3.2 Vibration Study . . . . . . . . . . . . . . . . . . . .29

3.3.3 Measurement of Torsional Spring Constants and Damping . . . . . 30

4.0 Theoretical System Model $\ldots \ldots \ldots \ldots 33$

4.1 Lumped Parameter, Two Degree-of-freedom Model . . . . . . . . . . . . 33

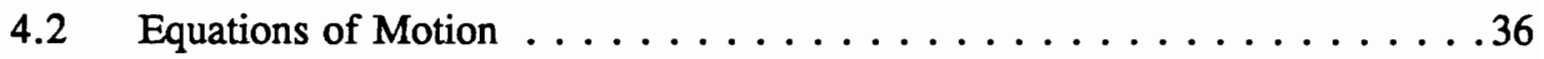

4.3 Theoretical Beam Acceleration . . . . . . . . . . . . . . . . 39

5.0 Experimental Results $\ldots \ldots \ldots \ldots \ldots \ldots \ldots \ldots$

$5.1 \quad$ Regimes of Frictional Behavior $\ldots \ldots \ldots \ldots \ldots \ldots \ldots \ldots \ldots$

5.1 .1 Fluorocarbon Elastomer $\ldots \ldots \ldots \ldots \ldots \ldots \ldots$

Characterization of friction induced vibration

Characterization of the frequency spectra

Friction/velocity relationship

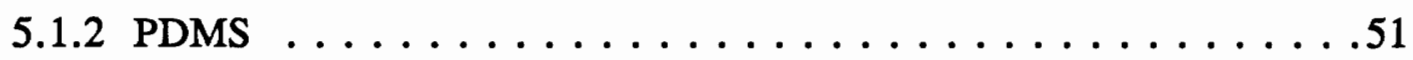

Characterization of friction 
Friction/velocity relationship

5.1 .3 Natural Rubber . . . . . . . . . . . . . . . . 51

Friction Behavior

Friction/velocity relationship

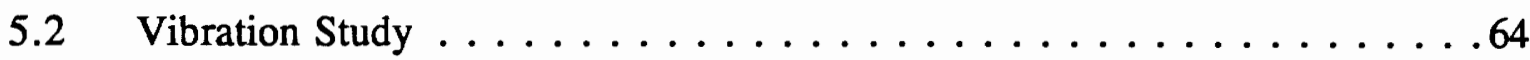

5.2.1 Friction results-Fluorocarbon $\ldots \ldots \ldots \ldots \ldots \ldots$

Effect of velocity and interference fit

Effect of system dynamics

5.2.2 Friction results-Natural rubber $\ldots \ldots \ldots \ldots \ldots \ldots \ldots \ldots$

Effect of transfer film

Effect of system dynamics

5.2 .3 Acoustic Results $\ldots \ldots \ldots \ldots \ldots \ldots \ldots \ldots \ldots$

Fluorocarbon

PDMS

Natural Rubber

5.3 Torsional Spring Constants and Damping Coefficients $\ldots \ldots \ldots$. . . 84

6.0 Discussion . . . . . . . . . . . . . . . . . .89

6.1 Friction Behavior . . . . . . . . . . . . . . . . . . . .89

6.2 Conditions Required For Noise Generation $\ldots \ldots \ldots \ldots$. . . . 94

6.3 Comparison of Simulated and Experimental Behavior . . . . . . . . . 97

6.3.1 Friction/velocity Results of Simulation . . . . . . . . . . .97

6.3.2 Comparison of Actual and Simulated Behavior . . . . . . . . 102

6.3.3 Comments Regarding the Validity of the Model . . . . . . . . 112

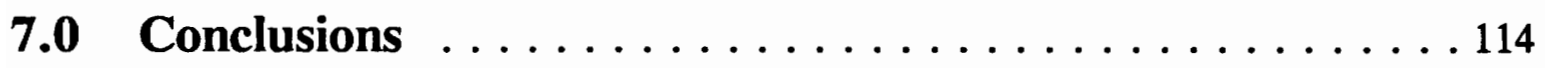


8.0 Recommendations for Further Study . . . . . . . . . 116

References . . . . . . . . . . . . . . . . . . . . 118

Appendix A. Theoretical Solution of Shaft Natural Frequencies 121

Appendix B. Calibration of Radial Potentiometer . . . . . . 126

Appendix C. Calibration of Torque Cell . . . . . . . . . 129

Appendix D. Calibration of Accelerometer . . . . . . . . 133

Appendix E. Calibration of Microphone . . . . . . . . 134

Appendix F. Listing of Lumped Parameter Model Program . . 135

Appendix G. Surface Temperature Model . . . . . . . . . . 139

Appendix H. Acoustic Response of the Apparatus . . . . . . . 146

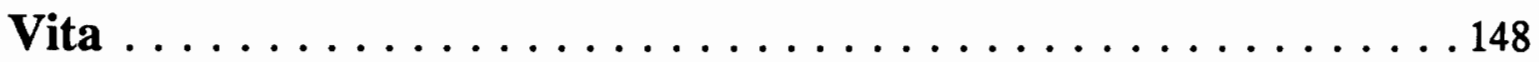




\section{List of Figures}

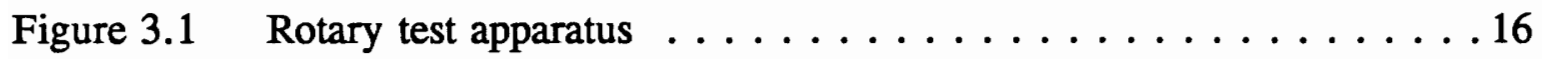

Figure 3.2 Clamping assembly providing variable interference fit $\ldots \ldots \ldots 16$

Figure 3.3 Theoretical natural vibration modes of beam bushing system $\ldots . .20$

Figure 3.4 The chemical structure of fluorel copolymer $\ldots \ldots \ldots \ldots$

Figure 3.5 The chemical structure of polydimethylsiloxane $\ldots \ldots \ldots 24$

Figure 3.6 The chemical structure of polyisoprene $\ldots \ldots \ldots \ldots \ldots 25$

Figure 4.1 Lumped parameter model of the torsional test apparatus . . . . . . 34

Figure 4.2 Free body diagram of forces acting on system $\ldots \ldots \ldots \ldots 37$

Figure 4.3 Free body diagram of system to determine theoretical stiffness . . . 40

Figure 5.1 Four types of friction behavior that were observed $\ldots \ldots \ldots 45$

Figure 5.2 Frequency content of different types of oscillations observed . . . . 49

Figure 5.3 Fluorocarbon friction torque/velocity relationships with $k_{B}=\infty \ldots 50$

Figure $5.4 \quad$ Stable sliding behavior of PDMS $\ldots \ldots \ldots \ldots \ldots \ldots \ldots \ldots$

Figure $5.5 \quad$ PDMS friction velocity relationships $\ldots \ldots \ldots \ldots \ldots \ldots$

Figure $5.6 \quad$ PDMS friction velocity relationships $\ldots \ldots \ldots \ldots \ldots \ldots$

Figure 5.7 Natural rubber friction behavior $\ldots \ldots \ldots \ldots \ldots \ldots$

Figure 5.8 Natural rubber friction behavior $\ldots \ldots \ldots \ldots \ldots \ldots \ldots \ldots$

Figure 5.9 Natural rubber friction behavior with transfer film $\ldots \ldots \ldots 58$

Figure 5.10 Clean surface of epoxy-painted shaft $\ldots \ldots \ldots \ldots \ldots \ldots$ 
Figure 5.11 Edge of natural rubber transfer film $\ldots \ldots \ldots \ldots 0$

Figure 5.12 Smeared natural rubber transfer film $\ldots \ldots \ldots \ldots \ldots$

Figure 5.13 Natural rubber friction/velocity relationships $\ldots \ldots \ldots \ldots 6$

Figure 5.14 Frequency content of friction torque with increasing velocity $\left(\delta=0.279 \mathrm{~mm}, k_{B}=2,214 \mathrm{Nm} / \mathrm{rad}\right) \ldots \ldots \ldots \ldots 6$

Figure 5.15 Frequency content of friction torque with increasing interference fit 67

Figure 5.16 Frequency content of friction torque with increasing velocity $(\delta=0.178 \mathrm{~mm})\left(\right.$ a) $k_{B}=2,214 \mathrm{Nm} / \mathrm{rad}$ and (b) $k_{B}=$ infinite $\ldots 69$

Figure 5.17 Frequency content of friction torque with increasing velocity $\left(\delta=0.279 \mathrm{~mm}, k_{B}=\infty\right) \ldots \ldots \ldots \ldots \ldots$

Figure 5.18 Frequency content of friction torque with increasing beam stiffness . 71

Figure 5.19 Natural rubber friction torque behavior $\left(k_{B}=2,214 \mathrm{Nm} / \mathrm{rad}, \delta\right.$ $=0.178 \mathrm{~mm}$ ) (a) torque time history and (b) autospectral density $\ldots 74$

Figure 5.20 Natural rubber friction torque behavior $(\omega=10 \mathrm{rad} / \mathrm{s}, \delta=0.127$ mm) (a) $k_{B}=2,214 \mathrm{Nm} / \mathrm{rad}$ and (b) $k_{B}=3,745 \mathrm{Nm} / \mathrm{rad} \ldots \ldots 75$

Figure 5.21 Torque, beam acceleration, and acoustic response during creak noise generated with the fluorcarbon elastomer $(\omega=1 \mathrm{rad} / \mathrm{s}$, $k_{B}=2,214 \mathrm{Nm} / \mathrm{rad}$, and $\left.\delta=0.381 \mathrm{~mm}\right) \ldots \ldots \ldots \ldots \ldots$

Figure 5.22 Torque, beam acceleration, and acoustic response during squawk noise generated with the fluorcarbon elastomer $(\omega=5 \mathrm{rad} / \mathrm{s}$, $k_{B}=2,214 \mathrm{Nm} / \mathrm{rad}$, and $\left.\delta=0.279 \mathrm{~mm}\right) \ldots \ldots \ldots \ldots$

Figure 5.23 Torque, beam acceleration, and acoustic response during squawk noise generated with the fluorcarbon elastomer $(\omega=5 \mathrm{rad} / \mathrm{s}$, $k_{B}=3,745 \mathrm{Nm} / \mathrm{rad}$, and $\left.\delta=0.279 \mathrm{~mm}\right) \ldots \ldots . \ldots . \ldots 80$

Figure 5.24 Torque, beam acceleration, and acoustic response during squeal noise generated with the fluorcarbon elastomer $(\omega=10 \mathrm{rad} / \mathrm{s}$, $k_{B}=3,745 \mathrm{Nm} / \mathrm{rad}$, and $\left.\delta=0.279 \mathrm{~mm}\right) \ldots \ldots \ldots . \ldots . \ldots 1$

Figure 5.25 Static deflection versus torque data with linear regression curve fit with 0.279 interference fit $\ldots \ldots \ldots \ldots \ldots . \ldots . \ldots 5$ 
Figure 5.26 Static deflection versus torque data with linear regression curve fit with 0.381 interference fit $\ldots \ldots \ldots \ldots \ldots$

Figure 6.1 Torque, displacement, and relative velocity during a slip event $\ldots 99$

Figure 6.2 Torque/velocity relationship during successive stick-slip cycles $\ldots 100$

Figure 6.3 Phase plane representation of stick-slip behavior $\ldots \ldots \ldots 101$

Figure 6.4 Torque, displacement, and relative velocity at the onset of harmonic oscillations . . . . . . . . . . . . . 102

Figure 6.5 Torque/velocity relationship during harmonic oscillations . . . . . 104

Figure 6.6 Phase plane representations of damped harmonic oscillations . . . 105

Figure 6.7 Torque, displacement, and relative velocity during a slip event $\ldots 106$

Figure 6.8 Simulated and experimental friction behavior in the time and

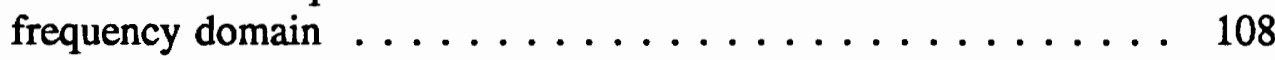

Figure 6.9 Simulated and experimental friction and system acceleration behavior in the time domain . . . . . . . . . . . . . . . 109

Figure 6.10 Effect of damping on friction induced vibration behavior $\ldots \ldots 111$

Figure A1 Torque acting on a rod element $d x \ldots \ldots \ldots \ldots \ldots \ldots$

Figure A2 Shaft torque and inertia torque $\ldots \ldots \ldots \ldots \ldots \ldots \ldots$

Figure B1 Calibration curve of the radial potentiometer $\ldots \ldots \ldots \ldots 128$

Figure $\mathrm{C} 1 \quad$ Calibration curve of the torque cell $\ldots \ldots \ldots \ldots \ldots \ldots$

Figure D1 Calibration and specification of the accelerometer . . . . . . 133

Figure G1 Constant surface heat flux between tow semiinfinite solids . . . 140

Figure G2 Semiinfinite solid with constant surface heat flux and transient

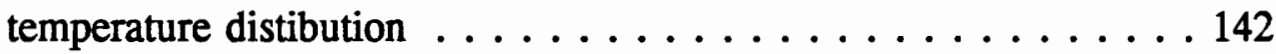

Figure G3 Temperature distribution due to constant temperature heat flux . . 145 
Figure H1 Acoustic frequency response function for (a) the $12.3 \mathrm{~cm}$ beam length condition, (b) the $9.2 \mathrm{~cm}$ beam condition, and (c) the fixed clamp condition . . . . . . . . . . . . . 147 


\section{List of Tables}

Table 3.1 Torsional natural frequencies of the shaft $\ldots \ldots \ldots \ldots$

Table 3.2 Theoretical torsional spring rates of the support structure $\ldots \ldots 18$

Table 3.3 Chemical composition of fluorocarbon cylinders $\ldots \ldots$. . . . . 24

Table 3.4 Chemical composition of polydimethylsiloxane $\ldots \ldots \ldots$

Table 3.5 Chemical composition of natural rubber cylinders . . . . . . . 26

Table 3.6 Test conditions for the friction/velocity study . . . . . . . . 29

Table 3.7 Test parameters for the vibration study $\ldots \ldots \ldots \ldots$

Table 3.8 Test parameters for the torsion spring constant measurements $\ldots 32$

Table 5.1 Transition velocity from stick-slip to quasi-harmonic oscillations . . 47

Table 5.2 Transition velocity from quasi-harmonic oscillations to steady

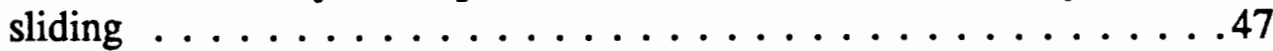

Table 5.3 Torsional spring rates of system and fluorocarbon elastomer $\ldots . .84$

Table 5.4 Percent error between theoretical and measured spring rates $\ldots \ldots 87$

Table 5.5 Torsional damping coefficients of system and fluorocarbon

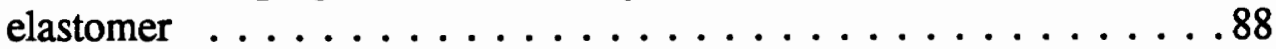

Table B1 Calibration data for the radial potentiometer . . . . . . . 127

Table $\mathrm{C} 1 \quad$ Calibration data for the torque cell . . . . . . . . . . . 130

Table G1 Thermal properties of 304 stainless steel and rubber $\ldots \ldots$. . . 143 


\subsection{Introduction}

This study investigates friction induced vibration that results in audible noise when elastomeric materials slide on hard painted steel surfaces. The origin and nature of the self-excited oscillations is studied between a shaft rotating in an elastomeric bushing such as that which supports the stabilizer bar in an automotive suspension system. The main thrust of this research was to determine the manner in which the system dynamics influence friction behavior, the friction mechanisms responsible for the self-excited oscillations, and to find sliding velocity/interference fit/material relationships leading to friction induced vibration and noise generation.

To accomplish the research goals it was necessary to fabricate a rotary test apparatus with variable system dynamics operating under a range of conditions. A digital measurement system was incorporated with the apparatus to measure the response of the system and to provide a means of numerically evaluating the unstable sliding behavior and audible noise.

Initially, it was necessary to characterize different types of friction phenomena and verify the operating conditions required for each. The friction/velocity relationships were determined experimentally for each material to establish if the kinetic friction was responsible for the sliding instability. This basic understanding of the friction behavior was extended by examining how the system input, the shaft velocity, and other system parameters including load and system dynamics influence the frequency content of 
friction. From these measurements it was possible to characterize and correlate system inputs with mechanical and acoustical responses of the system subjectively and quantitatively in terms of frequency content, vibration amplitude, and mean levels of oscillations.

To aid in developing a set of guidelines to predict conditions required to avoid frictional oscillations and acoustic noise generation, a discontinuous stick-slip model was developed. This simulation was used to illustrate the interaction between the system dynamics and other system parameters with the sliding behavior at the elastomer/shaft interface.

This thesis will develop and extend the interaction between the tribology and dynamics of a sliding pair during friction induced vibration. Section 2 is a review of previous research related to elastomeric friction and friction induced vibration. The investigation of system dynamics and acoustics during frictional vibration are also surveyed. The experimental equipment, instrumentation, materials, and procedure are described in depth in Section 3. In Section 4, a numerical stick-slip model is developed incorporating the inertia, stiffness, and damping of the elastomer and supporting structure. This model will be used to evaluate the interaction of system parameters and the sliding behavior. These parameters are experimentally determined and presented in Section 5. Also included in Section 5 are the results from the friction/velocity, vibration, and acoustic studies of the frictional oscillations. The results of Section 5 are interpreted in Section 6 and actual and simulated behavior using the model developed in Section 4 are compared. In Sections 7 and 8, conclusions and recommendations are drawn from 
experimental and theoretical investigations to summarize the cause of friction instability and noise generation and methods of avoiding it. 


\subsection{Review of the Literature}

\subsection{Friction Theories for Elastomers}

Although sliding friction between an elastomer and hard counterface behaves according to the basic laws of friction, factors influencing the mechanism of friction differ distinctly from metal-on-metal friction. Differences result from viscoelastic properties, mechanisms of adhesion, reduced hardness, melting temperature, and elastic modulus of the elastomer. Several theories have been developed to explain the mechanism of dry rubber friction on molecular and macroscopic levels, each with its critics. However, four points are generally agreed upon.

First, adhesion between the crosslinked chains of the elastomer and a hard surface is due to the formation and shearing of weak interfacial bonds. Part of the friction force results from the energy required to shear these bonds. Tabor [1], Roberts [2], and others agree this process requires a net loss of energy, dissipated as heat, during dry rubber friction.

Second, a contribution to the friction results when the energy of deformation is not fully recovered. Greenwood and Tabor [3] conducted friction experiments by ploughing a spherical metal slider over lubricated rubber. The rubber was lubricated in an attempt to observe the friction due to deformation and alleviate friction by other mechanisms. They noted that the friction increased with increasing normal pressure and that this correlated with the effect of hysteresis losses of the rubber. Schallomach [4] 
observed that soft elastomers sliding on Perspex form waves of detachment which traverse the interface. The energy required to buckle and unpeel the elastomer from the harder substrate is not fully recovered in a manner similar to the energy dissipated by shearing adhered junctions.

Third, the relative contribution of adhesion and associated deformation losses to the friction force depends on sliding velocity and temperature. The result is the well known viscoelastic peak when the coefficient of friction is plotted versus sliding velocity. Grosch [5] showed that the frictional behavior of elastomers depends on the temperature/velocity relationship of the viscoelastic properties. Grosch conducted sliding experiments at similar velocities but different temperatures. Using the Williams, Landel, \& Ferry (W.L.F.) transform, Grosch found the coefficient of friction was co-dependent on the sliding velocity and the temperature at the interface. The coefficient of friction is plotted versus the logarithm of the velocity which has been shifted by the amount $\log _{10} a_{T}$. Equation 2.1 is the W.L.F. transform into which the test temperature, $T_{T}$, and a reference temperature, $T_{s}$, are substituted to find the shift factor.

$$
\log a_{T}-\frac{-8.88\left(T_{T}-T_{S}\right)}{101.5+\left(T_{T}-T_{S}\right)}
$$

He showed the data collapsed on a single master curve for a given reference temperature and confirmed the viscoelastic nature of friction.

Finally, one would expect the surface roughness and hardness of the elastomer and hard substrate to affect friction force magnitude. Moore [6] explains that roughness is the primary factor which determines the real area of contact (and thus adhesion) when 
a normal load acts on an elastomer.

\subsection{Friction Induced Vibration}

Three classifications of frictional vibration have been reported in the literature. These are (1) stick-slip, (2) self-excited harmonic oscillations, and (3) vibration induced by random surface irregularities. Each of these frictional phenomena have been correlated with system vibration and noise generation. The nature of the surfaces in contact as well as operating conditions (normal load and sliding speed) dictate which type of vibration will occur if any.

Stick-slip motion, ideally characterized by a periodic sawtooth wave form, was observed for various sliding pair combinations including elastomer, metal, and glass $[7,8,9,10,11,12,16,17,18]$. Emphasis has been directed primarily to metal-on-metal sliding systems. Stick-slip sliding motion begins with no relative motion between the sliding pair, followed by a sudden slip at the interface. Ludema [7] provides an explanation of the intermittent motion with a one degree-of-freedom, lumped spring-mass model. The model consists of a mass resting on a stationary surface and the mass is connected by a spring to a prime mover. At time $t=0$, the velocity of the mass is zero and the velocity of the prime mover is equal to $V$. The motion of the prime mover causes the spring force to increase until it equals the static friction force, $F_{\text {satr }}$. Further motion of the prime mover will cause the spring force to exceed $F_{\text {saus }}$, and the mass will slip relative to the stationary surface. If the kinetic friction force, $F_{k i n}$, is equal to $F_{\text {stars }}$, 
the mass will slide steadily. However, if $F_{k i n}$ is less than $F_{\text {star }}$, the potential energy stored in the spring at $F_{\text {stat }}$ minus that at $F_{\text {kin }}$ causes the mass to slip and accelerate about the equilibrium position determined by $F_{k i n}$. Then the spring exerts a force on the mass which is less than that required to sustain sliding causing the mass to decelerate and stick when the velocity of the mass is zero.

Stick-slip friction is generally attributed to a difference in the static and kinetic coefficients of friction by researchers Bowden and Leben [8], Rabinowicz [9], and Brockley, et al $[10,11,12]$. Rabinowicz and Brockley assert that an additional requirement is the increase of $\mu_{\text {stat }}$ with time, during which the area of contact increases, thus allowing the support of a larger tangential load. Once the shear strength of the junctions is reached, decreased contact area or cohesive failure of the softer material causes a discontinuity in the forces acting on the supporting structure of the substrates. As the sliding surfaces accelerate to their new equilibrium state, $F_{k i n}$ opposes motion of the surfaces until the relative velocity returns to zero.

Several controlling factors influence the magnitude and frequency of stick-slip vibration. Increased sliding velocities elevate the rate of shear loading and thus the slip frequency. Rabinowicz [9] and Brockley, Cameron, and Potter [10] observed a reduction in stick amplitude for steel-on-steel systems with increased sliding velocity. After a critical velocity was reached, any further increase in sliding velocity produced steady sliding. Velocity variations can also dictate the amplitude and rate of slip. Material properties, including melting temperature of the metal, can also regulate the magnitude and frequency of stick-slip. Bowden and Leben's experiments show a 50-fold increase 
in slip amplitude for Wood's Alloy (melting point $70^{\circ} \mathrm{C}$ ) on steel as compared with molybdenum (melting point $2470^{\circ} \mathrm{C}$ ) on steel. Similar observations for elastomer sliding on metal or glass are not reported directly in the literature. However the temperature effect on the viscoelastic nature of adhesion may have a similar effect on stick amplitude.

If the difference between $\mu_{\text {stat }}$ and $\mu_{k i n}$ is small, or if the friction force varies inversely with velocity, self excited harmonic oscillations may occur for metals and elastomers. The development of frictional excitations may arise in both linear and nonlinear systems. Aronov, et al $[13,14,15]$, Rabinowicz [9], and others state that at sliding velocities which ensure a negative slope of the friction-velocity curve, negative damping will cause energy input to the sliding system. Energy input maintains or elevates the amplitude of the friction induced vibration. Brockley shows phase plane plots of velocity versus displacement. These plots indicate quasi-harmonic vibrations for steel-on-steel sliding, and show there are lower and upper limits of velocity where these vibrations can occur.

\subsection{Role of System Dynamics}

Theoretical and experimental investigations have been undertaken to suppress stick-slip and self-excited harmonic oscillations in metal-on-metal and elastomer-on-metal systems. These studies reveal that the dynamic characteristics of the supporting structure influence the amplitude and frequency of these friction induced vibrations. The level of system stiffness, damping, and mass or inertia dictate this. Less obvious effects arise 
from system geometric and modal constraints. Researchers have shown that energy feedback to the interface from vibrational modes in the supporting structure has been shown to incite unsteady sliding. Similarly, the addition of a normal degree of freedom with insufficient stiffness will provoke frictional fluctuations. Bulk properties of the sliding pair must be considered as well as the supporting structure.

Several investigations confirm correlation between natural resonances of the system and frequency of friction induced vibration for a variety of sliding configurations and materials including metals, elastomers, and ceramics. Kiryu, et al. [16], while observing ringing phenomena in automotive water pump seals, discovered frequency of torque fluctuations correspond to the fundamental frequency of the experimental rotating shaft system. They later learned [17] torque vibration was constrained by fundamental bending and torsional modes of the shaft during the stick period of the oscillation. During the slip portion, torque fluctuations resulted from free vibration of the stationary support structure. Bhushan [18] modeled propeller shaft bearings using a glass slider and Buna-N rubber stave. He discovered that stick-slip motion was coupled with mechanical resonances of the bearing supporting components.

In an effort to suppress vibration in sliding systems, several researchers varied system parameters while observing amplitude and frequency of friction induced vibration.

Rabinowicz [9] conducted sliding steel-on-steel experiments. He observed stickslip amplitude as a function of sliding velocity for various spring stiffnesses. His findings showed that stiffer configurations reduced stick-slip amplitude and that increased velocities diminished amplitudes abruptly. He attributed the need to slide through a 
critical distance (not allowed by stiffest springs used) before the static coefficient of friction drops to the lower kinetic value [9]. Similarly, Kiryu et al. [17] eliminated higher frequency vibration with increased spring rate. They observed that friction induced vibration for softer spring rates was more sensitive to changes in velocity and load.

Aronov, et al. [13] extended Rabinowicz's work to eliminate self-excited quasiharmonic oscillations for a steel-on-steel sliding configuration. They concluded that as stiffness increased, the frequency of vibration in the frictional direction increased and the range of steady state friction before self-excited oscillation occurs also increased.

Kato, et al. [19] were able to diminish amplitude of machine tool slideway vibration by decreasing the mass of the slider. This change effectively prevented the natural resonance of the system from exciting frictional oscillations that were inherent at the sliding interface. Kiryu, et al. [3] were able to eliminate higher frequency vibration by increasing the inertia of the rotating shaft system.

Brockley, Cameron, and Potter [10] conducted a study of the existence of a critical velocity when stick-slip vibrations appear to die out. They related $V_{\text {cris }}$ to the degree of damping in the system and the time and velocity dependent friction characteristics of the surfaces. Their theory revealed that the degree of damping in the system reduced amplitude of stick-slip vibration at any given surface velocity, and also reduced the maximum velocity at which vibration ceased.

Brockley and Ko [11] applied theory to experiment for quasi-harmonic frictional vibration. They demonstrated that sufficient increase in damping will suppress vibration 
over the entire velocity range in reasonable agreement with theory.

\subsection{Acoustics and Friction Induced Vibration}

Correlation between frictional characteristics produced during stick-slip motion and noise generation have been reported in the literature. An immediate drop in friction at the beginning of slip provides an exciting force which initiates system vibration and acoustic output. Methods of suppressing friction induced vibration discussed in Section 2.3 also seem to attenuate or eliminate acoustic output.

\section{Relationship of Noise Generation and System Natural Frequencies}

Bhushan [18] studied the fundamental mechanism of noise generation in water lubricated compliant bearings for marine propeller shafts. He discovered low frequency chatter was generated by frictional vibration when the lubrication film between the bearing and shaft deteriorated and the noise generated was due to mechanical resonances of the bearing support. Vibration generated squeal at the elastomer/glass interface. The squeal frequency was a function of the elastomer's elastic and shear modulus and the specimen thickness. Kiryu, et al. $[16,17,20]$ also found the relationship between ringing phenomena and frictional and resonant frequencies of the experimental system once fluid lubrication sliding conditions transitioned to non fluid.

Automotive braking systems are a tribological system with undesirable squeal noise. Investigators consider the primary brake components as well as the friction materials. Schwartz, et al [21] describe methods of "quiet" brake design considering the 
rotor and pad assembly. Their approach stems from experimental modal analysis which reveals squeal noise frequency generally corresponds to one of the rotor natural frequencies. They suggest that stiffness and vibrational modes of the pad backing plate influence the tendency of squeal noise.

Earles and Soar [22] investigated brake system squeal using a pin-on-disc configuration. They varied pin contact angle, stiffness of the pin support, radius of pin contact, and disc thickness. Theory and experiment revealed that disc mode shapes that were excited when the pin support vibrated torsionally and produced squeal were not excited when the support vibrated in a translational mode.

Relationship of Noise and Load, Velocity, Mass, Temperature, and Surface Roughness

Having identified the cause of noise generation, Kiryu, et al. sought to identify the relationship between the ringing phenomena and contact load, sliding velocity, and lubricant temperature. They determined that lower frequency ringing varied with these parameters plus the torsional stiffness of the shaft. Frequency spectra of noise and torque variations changed in relation to shaft speed. As shaft speed and surface load increased, the amplitude of ringing increased. Increased shaft speed also resulted in shorter stick periods, causing an impulse to the system. The longer slip period following allowed the system to vibrate freely giving more time for acoustic emission. Higher frequency ringing was associated with torsional stiffness and mass moment of inertia of rotating parts but was not affected by speed variations. Raising the temperature of the lubricant broadened the sliding velocity range in which stick-slip and noise generation occurred. 
Bhushan [18] noted several conditions which encouraged squeal and chatter. Roughened counterfaces were more likely to squeal. His explanation was that smooth sliding surfaces stick, and thus require a greater build up of force in the sliding direction in order to slip. Fewer sticks will occur and only lower frequency resonances will be excited producing low frequency chatter. Squeal was generated at low load and high speeds due to high frequency of slip. Chatter had greater acoustic intensity and resulted at high loads and all speeds because larger stick amplitudes excited the supporting structure.

\section{Relationship of Noise Generation to Material}

Investigators have also attempted to correlate acoustic noise generation with materials and material properties. Othman, et al. [23], while investigating the relationship between surface roughness, contact load, and frictional sound properties, concluded that sound pressure levels increased with increasing roughness and load for steel, brass, and aluminum. Note that, for each sliding system, frequency of acoustic emission did not vary with load. The dominating frequency varied linearly with the sonic speed of each material. Symmons and McNulty [24] also studied frictional sound properties. They investigated the correlation between vibration of the stick-slip type and noise generation. They found material pairings having an immediate drop at the start of the stick period were able to give an exciting force for the onset of noise generation.

Previous research has shown that many factors contribute to the sliding behavior and the character of self-excited oscillations. In this investigation the relative 
contribution of adhesion and deformation terms will be examined. It will be necessary to identify the velocity dependence of frictional oscillations for various degrees of normal load and system stiffness. Finally, it will be necessary to examine the friction and acoustic behavior to see if they are dominated by vibration modes of the system. 


\subsection{Experimental Technique}

\subsection{Description of Apparatus}

A test apparatus was designed and fabricated providing rotary sliding motion between an epoxy painted shaft and a concentric elastomeric isolator. The apparatus was used to measure the friction force and system response of the sliding pair as well as the acoustical characteristics associated with the friction induced vibration causing the noise generation.

\subsubsection{Configuration}

The conceptual design is illustrated in Figure 3.1. The components of the test apparatus and their functions are as follows:

The primary shaft turns with constant velocity in the bushing specimen. The shaft is supported by two self-aligning, isolated, pillow block bearings. The shaft was manufactured from Schedule 80, 0.5" ID, 304 stainless tubing to provide enough flexibility for strain measurements while maintaining strength requirements. It was necessary to ensure the resonant frequencies of the primary shaft were much higher than the friction induced vibration. The range of natural frequencies were determined for the fixed-free and fixed-fixed boundary conditions. It was assumed the actual frequencies would fall within this range. The torsional modes of vibration for fixed-free and free- 


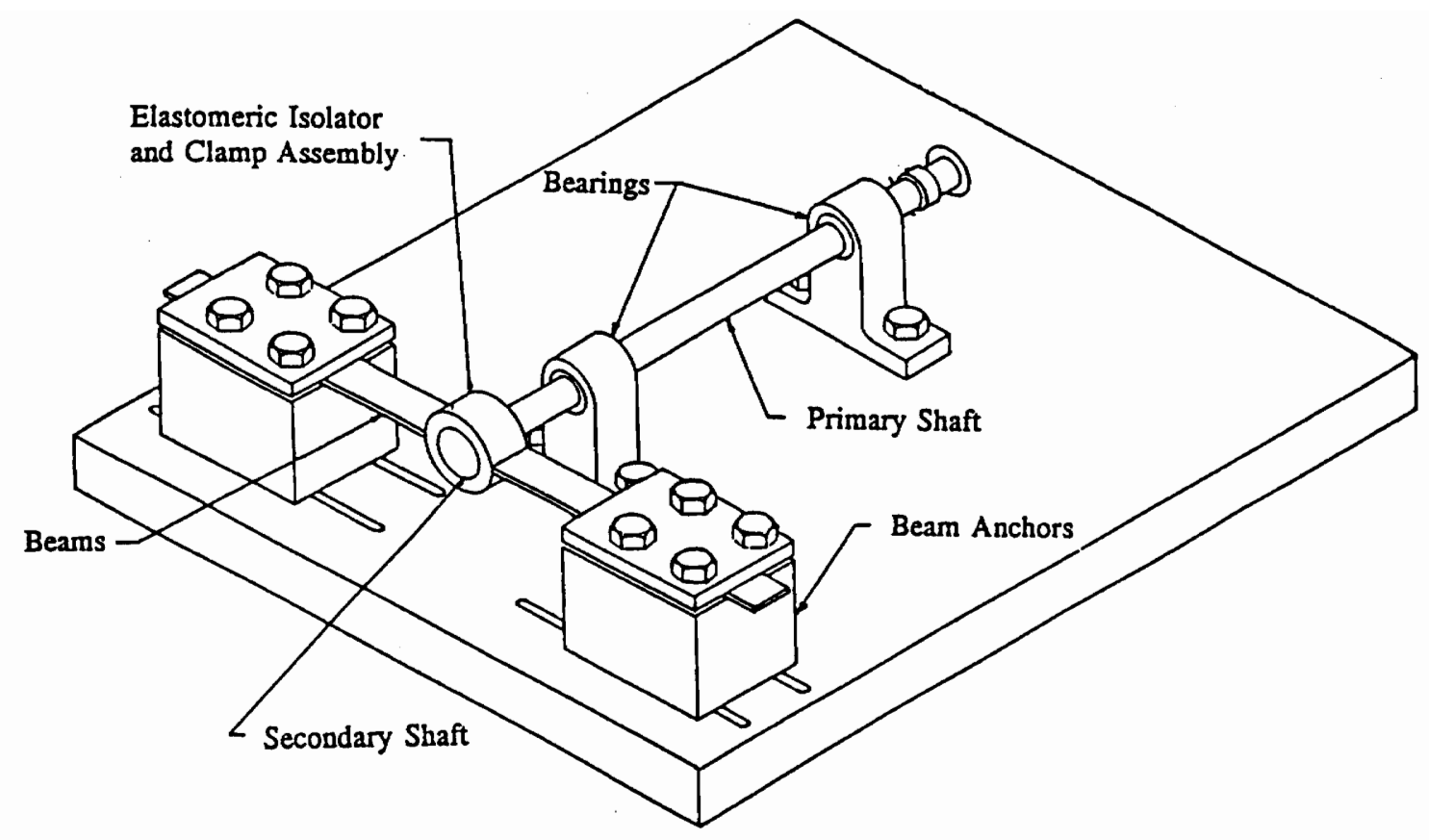

Figure 3.1 - Rotary test apparatus

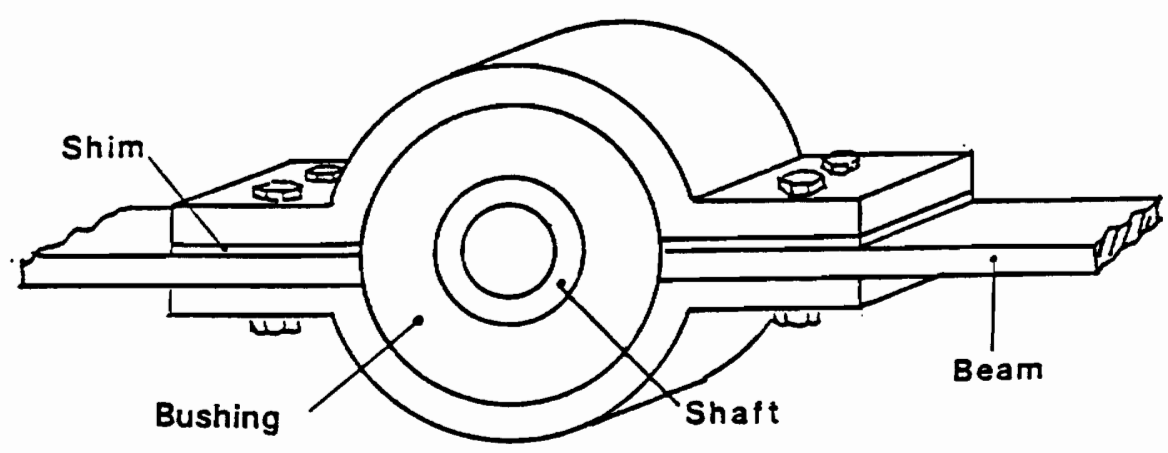

Figure 3.2 - Clamping assembly providing variable interference fit and coupling between the bushing and beams. 
free boundary conditions are listed in Table 3.1. The resonant frequencies are derived in Appendix A.

Table 3.1. Torsional natural frequencies of the primary and secondary shaft.

\begin{tabular}{|c||c|c||}
\hline Mode & Fixed-Free & Free-Free \\
\hline \hline 1 & $1568 \mathrm{~Hz}$ & $8131 \mathrm{~Hz}$ \\
\hline \hline 2 & $8462 \mathrm{~Hz}$ & $16262 \mathrm{~Hz}$ \\
\hline \hline 3 & $16433 \mathrm{~Hz}$ & $24393 \mathrm{~Hz}$ \\
\hline
\end{tabular}

A replaceable secondary, hollow shaft was pressfit and pinned at the end of the primary shaft. The outer diameter of the shaft was $3 \mathrm{~cm}$. The epoxy coated steel secondary shaft was manufactured from a 1988/1989 Ford Crown Victoria stabilizer bar. Original sections of the stabilizer were used to maintain the actual surface chemistry and roughness conditions.

The elastomeric bushing was clamped to the specimen mount with the clamp assembly as shown in Figure 3.2. Clamping the bushing produced a radial deflection and a normal contact pressure at the interface. The load was varied by adjusting shim thickness between the clamp flanges. This changed the interference fit between the bushing and the shaft. The elastomer was glued to the interior of the clamp housing to prevent slip between the elastomer and clamp while torque was transmitted to the beams.

Two stainless steel beams were attached to the bushing to provide an interactive system for the friction induced vibrations to excite and to act as radiating surfaces for 
acoustical energy. Active beam lengths were adjusted by securing the beam anchors at their minimum and maximum distances from the shaft to effectively increase (or decrease) beam stiffness. The torsional beam stiffness, $k_{B}$, is derived analytically later in Section 4.3 as the ratio of applied torque to the angular displacement of the clamp assembly. The beams were modelled as cantilevered beams with applied end load and end moment. Beam lengths of 9.2 and $12.3 \mathrm{~cm}$ were used for testing. This provided control of system stiffness and natural frequencies while observing friction and acoustic output.

To observe friction behavior without transmission of beam vibration to the interface, the beams were removed and the clamp was rigidly fastened to the baseplate. The torsional stiffness for this case is very large compared to the 9.2 and $12.3 \mathrm{~cm}$ beam length conditions. Table 3.2 lists the torsional support stiffness for these three stiffness conditions.

Table 3.2. Theoretical torsional spring rates of the support structure.

\begin{tabular}{||c|c||}
\hline $\begin{array}{c}\text { Beam Length } \\
{[\mathrm{cm}]}\end{array}$ & $\begin{array}{c}\text { Torsional Stiffness } \\
{[\mathrm{Nm} / \mathrm{rad}]}\end{array}$ \\
\hline \hline 12.3 & 2,214 \\
\hline 9.2 & 3,745 \\
\hline 0 & $\infty^{+}$ \\
\hline
\end{tabular}

'Very large compared with other stiffness conditions

The natural frequencies of the beam/clamp system were determined analytically via the transfer matrix method. The following modeling assumptions were made: 
(1) Beams and clamps are modeled as sections with uniform cross-section and Young's modulus.

(2) Clamps and bushing have concentrated mass and rotary moment of inertia.

(3) Bushing has a lateral bearing stiffness of $3500 \mathrm{lb} /$ in and torsional spring constant of $750 \mathrm{Nm} / \mathrm{rad}$.

(4) Beams have continuous distributed mass.

(5) Beam anchors provide infinite stiffness.

The first four modes of vibration are tuned in the range of interest-140-2100 Hz. Higher mode frequencies shown in Figure 3.3 are strong functions of beam length.

In addition to serving as a mounting surface, the baseplate acts as an acoustic radiator when excited by vibration transmitted through the beam anchors from the beams. Isolators for the beam anchors were fabricated to determine whether attenuating transmitted vibration to the baseplate affected acoustic output.

\subsubsection{Instrumentation and Data Acquisition.}

Measurements of angular velocity, frictional vibration, audible noise, and system response were possible using the following instrumentation:

A ten-turn radial potentiometer provided a measure of shaft angular displacement. Angular velocity was found from its time derivative. The potentiometer retained its linearity well above the $90^{\circ}$ displacement test range. The calibration and instrumentation for this signal are Appendix B.

Strain gages mounted on the primary shaft measured frictional torque. Since two 


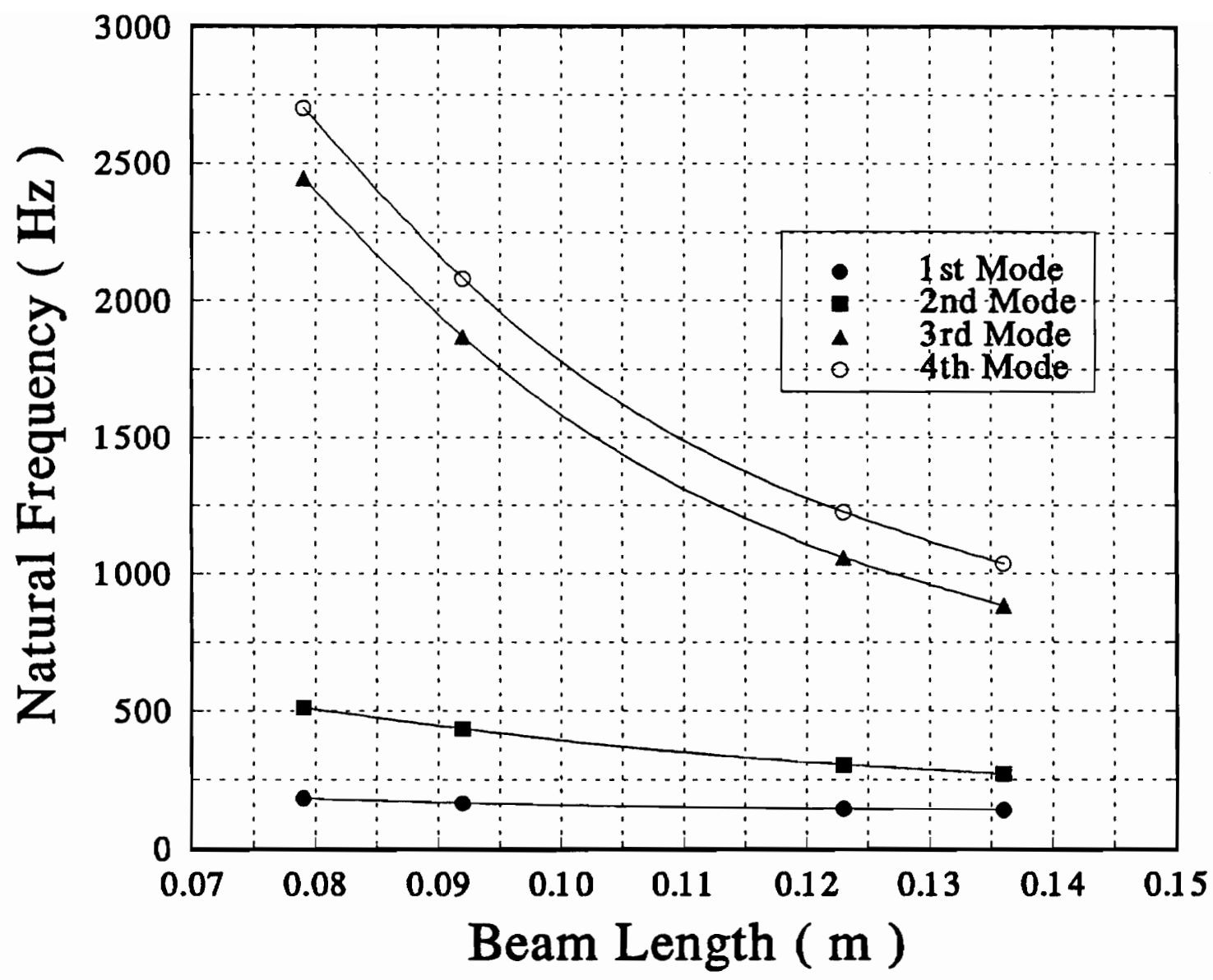

Figure 3.3 - Theoretical natural vibration modes of beam/bushing system. 
perpendicular, 45-degree helixes define the principle stress directions for a circular shaft subjected to pure torsion, two $45^{\circ}$-rosettes (four strain gages) were used. The gages were wired in a Wheatstone bridge configuration and could detect positive and negative torques. Transient friction force frequencies were well below the maximum frequency response for this type of transducer. Linearity was maintained through the torque range of interest to $\pm 40 \mathrm{Nm}$. The calibration and instrumentation for this transducer are in Appendix C.

Piezoelectric accelerometers placed on the beams and plate sensed response of the beam system. A 1:1 charge amplifier energized the transducer. Resonant frequency of the accelerometer was measured at $70 \mathrm{kHz}$. Specifications and calibration are in Appendix D.

A $1 / 2$-inch microphone sensed audible noise from the interface, beams, and baseplate. The microphone was suspended from the ceiling to isolate it from apparatus vibration. Calibration and instrumentation for this transducer are in Appendix E.

All signals were sent through low-pass filters for anti-aliasing purposes. Signals were captured on a Data Translation DT2821-F-16E digital acquisition board. The board employs 12-bit A/D converters with quantization error of $\pm 0.625 \mathrm{mV}$ for a input range of $\pm 1.25 \mathrm{~V}$. Each signal voltage was multiplied by the transducer's constant gain factor and sampled at $30 \mathrm{kHz} /$ channel.

\subsubsection{Operation.}

The apparatus operates in the following manner. An angular displacement caused 
by manual rotation of the primary shaft (at a specified velocity) initiates motion between the secondary shaft and bushing. The tangential friction force at the interface deflects the bushing and clamps thus storing potential energy in the elastomer and the beams. When the bushing slips at the interface, the beam system will vibrate at its natural frequencies causing the bushing and clamp to rotate about the shaft until the vibrations damp out. Noise, if any, will be generated from the interface, the beams, and the baseplate. In the sections that follow, an understanding of the functions and the dynamical interactions between the individual components of the apparatus is required.

Although the placement of the elastomer bushing was briefly mentioned in this section, a more extensive description of the chemical formulation and mechanical properties will aid in understanding the tribological and dynamical behavior of each material. This will be the topic of the next section. 


\subsection{Material Selection}

Three different elastomers were chosen for this investigation. The elastomers represent a broad range of mechanical and chemical properties including adhesion, abrasion resistance, tensile strength, hardness, tear strength, chemical resistance. Two of the materials were special purpose elastomers and one was natural rubber.

\subsubsection{Fluorocarbon Elastomer}

A fluorocarbon was investigated first. It has the highest elastic modulus, hardness, and strength of the materials tested. This particular fluorocarbon is a Fluorel which is a copolymer of vinylidene fluoride $\left(\mathrm{CH}_{2} \mathrm{CF}_{2}\right)$ and hexafluoropropylene $\left(\mathrm{C}_{3} \mathrm{~F}_{6}\right)$. Its structure is shown in Figure 3.4 .

vinylidene fluoride:

$\underset{\mathbf{H}}{\mathbf{C}}=\underset{\mathrm{F}}{\mathbf{C}}$

hexafluoropropylene:

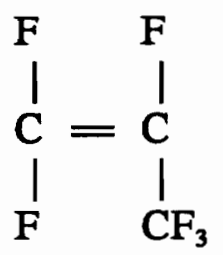

Figure 3.4 - The chemical structures of Fluorel Copolymer.

The materials were mixed and cured by Ford. The recipe is shown in Table 3.3. The cylinders were molded at $160^{\circ} \mathrm{C}$ for 30 minutes. 
Table 3.3. Chemical composition of fluorocarbon cylinders.

\begin{tabular}{||l|c|}
\hline Fluorocarbon Cylinders & $\mathrm{pphr}^{\mathrm{s}}$ \\
\hline \hline Fluorel FT-2481 Fluoroelastomer & 100 \\
\hline $\mathrm{MgO}$ & 3 \\
\hline $\mathrm{CaOH}$ & 6 \\
\hline $\mathrm{N} 990$ Carbon Black & 30 \\
\hline DuPont Curative 20 & 3 \\
\hline DuPont Curative 30 & 4 \\
\hline
\end{tabular}

"pphr is the abbreviation for parts per hundred relative

\subsubsection{Polydimethylsiloxane}

Polydimethylsiloxane (PDMS) was chosen because of its unique molecular structure and mechanical properties. PDMS samples were soft, had less strength, more flexibility, and possessed good vibration damping characteristics. PDMS absorbs vibrational energy over a wide temperature range. It also has excellent non-stick/nonadhesive properties [25]. The $\mathrm{Si}-\mathrm{O}$ backbone provides a saturated structure resistant to ozone and oxidation attack. The structure is shown in Figure 3.5.<smiles></smiles>

Figure 3.5 - The chemical structure of PDMS. 
The cylinders were molded at $160{ }^{\circ} \mathrm{C}$ for 30 minutes and then post cured for 24 hours at $200{ }^{\circ} \mathrm{C}$. The recipe for PDMS is shown in Table 3.4.

Table 3.4. Chemical Composition of PDMS.

\begin{tabular}{||l|c|}
\hline PDMS Cylinders & pphr \\
\hline PDMS & 100 \\
\hline Perkadox 14 & 1 \\
\hline Hi Sil 233 & 10 \\
\hline
\end{tabular}

\subsubsection{Natural Rubber}

The last material to be experimentally evaluated was a Natural rubber compound. Natural rubber was chosen as a basis for comparison with the other special purpose materials that were evaluated. This material provides intermediate Shore A hardness and tensile strength properties as compared to the fluorocarbon and the PDMS. The chemical structure shown in Figure 3.6 is similar to polyisoprene minus chemical impurities.<smiles>CCCC(C)C</smiles>

Figure 3.6 - The chemical structure of Polyisoprene.

The natural rubber cylinders were mixed and cured by The Gates Rubber Company. The 
recipe is shown in Table 3.5.

Table 3.5. Chemical composition of Natural rubber cylinders.

\begin{tabular}{||l|c||}
\hline Natural Rubber Cylinders & pphr \\
\hline \hline Natural Rubber & 100 \\
\hline N550 Carbon Black & 50 \\
\hline Zinc Oxide & 5 \\
\hline Stearic Acid & 2 \\
\hline Aminox & 1.15 \\
\hline Amax & 0.63 \\
\hline Rubbermaker & 0.10 \\
\hline \hline
\end{tabular}




\subsection{Experimental Procedure}

The experimental work was divided into three phases to investigate various physical conditions contributing to friction induced vibration and noise generation. The first phase was undertaken to determine which types of frictional phenomena occurred and at what shaft velocities they were encountered. The relative effects of system stiffness and interference fit were also examined. The second phase was a vibration study to observe how system dynamics influenced frictional character while constant velocity and pressure were maintained. Phase three provided a measure of the static torsional stiffness of the elastomer and beams and a measure of system damping. These mechanical properties could then be related to noise generation and would be incorporated into a computer model simulating vibratory motion and forces at the interface.

\subsubsection{Friction/Velocity Study}

\section{Regimes of Frictional Behavior}

This study was conducted to determine the possible types of vibration induced, such as stick-slip or harmonic oscillations for each elastomer. Thus, at constant pressure and beam stiffness, changes in friction and beam acceleration behavior were noted as a function of velocity. At each combination of beam stiffness and interference fit, the shaft was rotated at constant velocity. The transition velocity, $V_{\text {crill }}$, was noted when frictional vibration changed from one type to another. Transition velocity, $V_{\text {criz } 2}$, was noted when 
frictional oscillations of any type ceased.

Initially, friction regimes were determined subjectively through observation of changes in the audible noise content. Then the friction torque, the beam acceleration, and the angular displacement time histories were acquired digitally at $30 \mathrm{kHz}$. Tests were repeated as necessary until the velocities of transition were confirmed.

\section{Effect of System Stiffness on $V_{\text {crit }}$}

To determine whether system dynamics affected the types of vibration observed or the transition velocities, the beam stiffness, $k_{B}$, was increased from $2,214 \mathrm{Nm} / \mathrm{rad}$ to $3,745 \mathrm{Nm} / \mathrm{rad}$. This was accomplished by shortening the beam lengths from $12.3 \mathrm{~cm}$ to $9.2 \mathrm{~cm}$ while maintaining constant pressure. The beam elements were then removed from the system and the clamp was rigidly fastened to the base plate to prevent clamp vibration. Changes in friction behavior were observed without the stiffness or inertia of the system affecting the torque at the elastomer/shaft interface.

\section{Effect of Interference fit}

The load was then changed via constant strain by adding (or removing) $0.127 \mathrm{~mm}$ shims between the clamp flanges. Before mounting the clamp assembly on the secondary shaft, the ID of the compressed bushing was measured with a dial caliper. This provided a measure of the interference fit between the compressed bushing specimen and the secondary shaft. Transitions from one friction regime to another were recorded as a function of velocity.

\section{Summary of Test Conditions}

Operating conditions between the elastomer and secondary shaft are summarized 
in Table 3.6 with regard to material, beam stiffness, and interference fit between the OD of the shaft and the ID of the clamped bushing. All of the combinations were tested.

Table 3.6. Test conditions for the Friction/Velocity study.

\begin{tabular}{||c|c|c||}
\hline $\begin{array}{c}\text { Elastomeric } \\
\text { Material }\end{array}$ & $\begin{array}{c}\text { Beam Stiffness } \\
{[\mathrm{Nm} / \mathrm{rad}]}\end{array}$ & $\begin{array}{c}\text { Interference Fit } \\
{[\mathrm{mm}]}\end{array}$ \\
\hline \hline Fluorocarbon & $2,214,3,745, \infty$ & $0.179,0.278,0.381$ \\
\hline PDMS & $2,214, \infty$ & $0.254,0.381$ \\
\hline Natural Rubber & $2,214,3,745$ & .127 \\
\hline
\end{tabular}

\subsubsection{Vibration Study}

This phase of the investigation was undertaken to characterize and correlate frictional, system, and acoustic vibration for various materials. This was done to determine how system dynamics interact with load and velocity to affect the character of friction and the noise produced. The measurement of changes in vibration amplitude and frequency would allow the investigator to determine whether friction was controlled by modal frequencies via system feedback to the interface.

For each material tested, shaft displacement, frictional torque, beam acceleration, and acoustic pressure were captured digitally at $30 \mathrm{kHz}$ per channel for shaft displacements of $0.5-3 \mathrm{rad}$. Measurements were acquired at velocities of approximately $1.0,2.5,5.0,7.5$, and $10.0 \mathrm{rad} / \mathrm{s}$. The same three stiffness conditions used in the Friction/Velocity study were used for these tests. Any pressure (in $0.127 \mathrm{~mm}$ 
increments) at which noise was produced were tested at the angular velocities mentioned above. A summary of the test conditions is included in Table 3.7. All of the combinations were tested.

Table 3.7. Test parameters for the Vibration Study.

\begin{tabular}{||c|c|c|c||}
\hline $\begin{array}{c}\text { Elastomeric } \\
\text { Material }\end{array}$ & $\begin{array}{c}\text { Angular Velocity } \\
{[\mathrm{rad} / \mathrm{s}]}\end{array}$ & $\begin{array}{c}\text { Beam Length } \\
{[\mathrm{cm}]}\end{array}$ & $\begin{array}{c}\text { Interference Fit } \\
{[\mathrm{mm}]}\end{array}$ \\
\hline \hline Fluorocarbon & $1.0,2.5,5.0,7.5,10.0$ & $12.3,9.2,0$ & $0.178,0.279,0.381$ \\
\hline Natural Rubber & $1.0,2.5,5.0,7.5,10.0$ & $12.3,9.2$ & 0.127 \\
\hline
\end{tabular}

\subsubsection{Measurement of Torsional Spring Constants and Viscous Damping Coefficients}

This investigation had three objectives. The first was to produce static torque versus deflection curves to determine experimentally the torsional spring rate of the elastomer, $k_{E}$, and the effective torsional spring rate, $k_{E F F}$, of the entire beam/bushing system. The beam stiffness at a given beam setting could then be determined using the relation

$$
\frac{1}{k_{B}}-\frac{1}{k_{E F F}}-\frac{1}{k_{E}}
$$

and compared with theoretical values. The second goal was to establish the linearity of $k_{E}$ and $k_{E F F}$. The third goal was to determine $k_{E}$ as a function of pressure. This permitted determination of the shear and Young's modulus, $G$ and $E$, as a function of interference fit or pressure. 
Since the resolution of the radial potentiometer was too large to make the necessary angular measurements, a Talysurf surface profilometer was used to measure the angular displacement. A flat was glued to the end of the secondary shaft with one edge of the flat coincident with the diameter of the shaft. This provided a surface normal to the stylus of the profilometer. Small angular deflections and constant radius were assumed. The radial deflection $\theta_{1}$ of the interface was determined by dividing the linear deflection of the Talysurf stylus by the radius from the shaft centerline to the location of the stylus.

The Talysurf was calibrated using a roughness standard. The standard has three grooves nominally $2.5 \mu \mathrm{m}$ deep. The gain of the Talysurf amplifier was $55.5 \mu \mathrm{m} / \mathrm{V}$ at $500 \mathrm{x}$ magnification. Using a radius of $5.55 \mathrm{~mm}$ from the shaft center made the sensitivity of the measuring system equal to $0.01 \mathrm{rad} / \mathrm{V}$.

Static deflection and torque were collected for the three stiffness cases and 0.279 and $0.381 \mathrm{~mm}$ interference fits. The fixed case provided a measure of the torsional stiffness of the elastomer and measurements at the 12.3 and $9.2 \mathrm{~cm}$ beam length settings provided a measure of the effective torsional spring rate of the beam and elastomer acting in series. Data was collected until slip occurred. A summary of test conditions is shown in Table 3.8.

System damping coefficients were found for the same conditions (beam length and interference fit in Table 3.8. These values could be used to simulate the viscous damping of the two-degree-of freedom model. This was accomplished by using a modal hammer to excite the first torsional mode of vibration of the system. The second mode 
Table 3.8. Test parameters for the torsional spring constant measurements

\begin{tabular}{|c|c|c||}
\hline $\begin{array}{c}\text { Elastomeric } \\
\text { Material }\end{array}$ & $\begin{array}{c}\text { Beam Length } \\
{[\mathrm{cm} \mathrm{]}}\end{array}$ & $\begin{array}{c}\delta \\
{[\mathrm{mm}]}\end{array}$ \\
\hline \hline Fluorocarbon & 12.3 & 0.279 and 0.381 \\
\cline { 2 - 3 } & 9.2 & 0.279 and 0.381 \\
\hline
\end{tabular}

The first torsional mode of vibration is the second vibration mode of the system shown in Figure 3.3. The damped response of the system was acquired digitally. Anti-aliasing filters ( $600 \mathrm{~Hz}$ cut-off) eliminated higher mode frequencies. 


\subsection{Theoretical System Model}

A lumped parameter model of the torsional sliding system was developed to more fully understand the interaction of the dynamic characteristics, the amplitude and frequency of friction induced vibration, and the damped oscillations of the system. The influence of system parameters, load, and velocity on friction behavior is to be compared with experimental data acquired from the test apparatus. The simulation allows the identification of friction mechanisms during stick-slip and the nature of the kinetic friction during the slip cycle. Theoretical prediction of second mode beam vibration was correlated with frictional vibration as an indicator of incipient noise. In Section 4.1, the apparatus is modeled as a two degree-of-freedom system. The discontinuous equations of motion are derived in Section 4.2. In Section 4.3 the torsional stiffness of the supporting structure is derived. It will be used to measure the accuracy of the experimental torsional spring constants of the elastomer and beams.

\subsection{Lumped Parameter, Two Degree-of-freedom Model}

The lumped parameter model is shown in Figure 4.1. The mass moments of inertia, $J_{E}$ and $J_{C}$, account for the rotary inertia of the elastomer and clamp. $\theta_{1}$ denotes the angular displacement of the bushing counterface and $\theta_{2}$ represents the angular displacement of the clamp assembly. The fundamental natural frequencies observed in experiment were used with system spring rates to back solve the eigenvalue problem in 


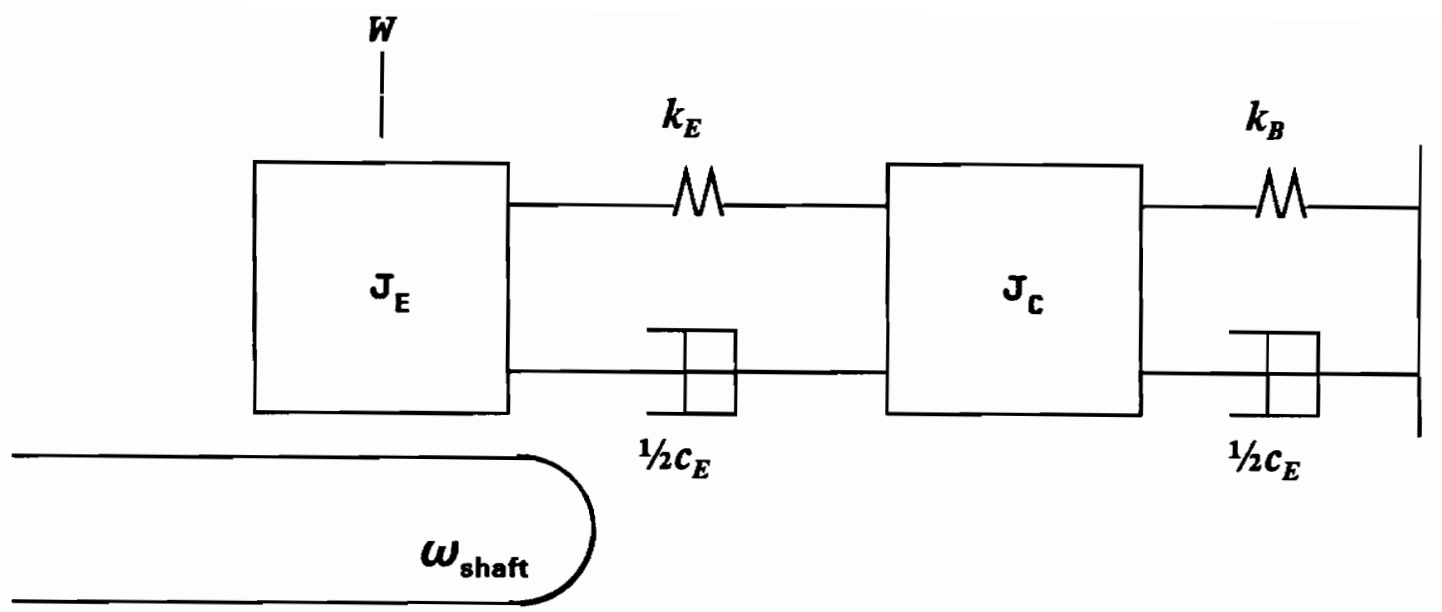

where: $\quad J_{E}=$ mass moment of inertia of the elastomer bushing

$J_{C}=$ effective mass moment of inertia of clamp, elastomer, and beam

$k_{E}=$ torsional spring rate of elastomer

$c_{E}=$ torsional damping of system

$k_{B}=$ torsional spring rate of beams

$\omega=$ shaft angular velocity

$W=$ load due to interference fit

Figure 4.1 - Lumped Parameter Model of the Torsional Test Apparatus. 
order to obtain the lumped inertias. The damping of the system was lumped evenly between the elastomer and beams since the relative contribution of each was not known. It was assumed $J_{E}$ is connected to the mass moment of inertia of the clamp, $J_{C}$, by the elements $k_{E}$ and $1 / 2 c_{E}$ representing the torsional spring rate and damping of the elastomer. The torsional stiffness and damping from clamp to ground is taken into account by $k_{B}$ and $1 / 2 c_{E}$. A theoretical derivation of the system parameter, $k_{B}$, is included in Section 4.3. The shaft surface rotating at angular velocity $\omega$ (modelled as the belt drive) is the dynamic input to the system. 


\subsection{Equations of Motion}

The model incorporates two sets of differential equations governing motion. The first set is relevant when the bushing is stuck implying $\dot{\theta}=\omega_{\text {shaff }}$. These equations govern motion throughout the stick phase until the forces in the elastomers spring and damper elements equal a critical torque, $T_{c r i t}$ which instigates slip. The value of $T_{c r i t}$ is derived from torque data at the specific operating conditions from each experiment.

The forces acting in the system are shown in Figure 4.2(a). While the bushing is stuck, the initial conditions are

$$
\theta_{1}(0)-0, \dot{\theta}_{1}(0)=\omega_{\text {shaft }}, \theta_{1}-\omega t
$$

Solving the equations of motion for the rotary mass moment $\mathrm{J}_{\mathrm{E}}$

$$
\sum T=J_{E} \ddot{\theta}_{1}: 0=T_{\text {shaft }}-\left[\left(\frac{1}{2} c_{E}\left(\dot{\theta}_{1}-\dot{\theta}_{2}\right)+k_{E}\left(\theta_{1}-\theta_{2}\right)\right]\right.
$$

and the equation for mass moment $\mathrm{J}_{\mathrm{C}}$ is

$$
\sum T=J_{C} \ddot{\theta}_{2}: J_{C} \ddot{\theta}_{2}=\left[c_{E}\left(\frac{1}{2} \dot{\theta}_{1}-\dot{\theta}_{2}\right)+k_{E}\left(\theta_{1}-\theta_{2}\right)-k_{B} \theta_{2}\right]
$$

The second order differential equation (4.4) is then rewritten for solution until slip occurs as

$$
\ddot{\theta}_{2}-\frac{1}{J_{C}}\left[c_{B}\left(\frac{1}{2} \dot{\theta}_{1}-\dot{\theta}_{2}\right)+k_{B}\left(\theta_{1}-\theta_{2}\right)-k_{B} \theta_{2}\right]
$$

Once $T_{\text {shaft }}=T_{\text {cris }}, \dot{\theta}_{1}$ will no longer equal $\omega$. The constant kinetic frictional torque, $T_{\text {kin }}$, will be the equilibrium torque about which $J_{E}$ and $J_{C}$ will oscillate until $\dot{\theta}_{1}$ again equals $\omega . T_{\text {kin }}$ is obtained from the mean value of torque data at the operating 


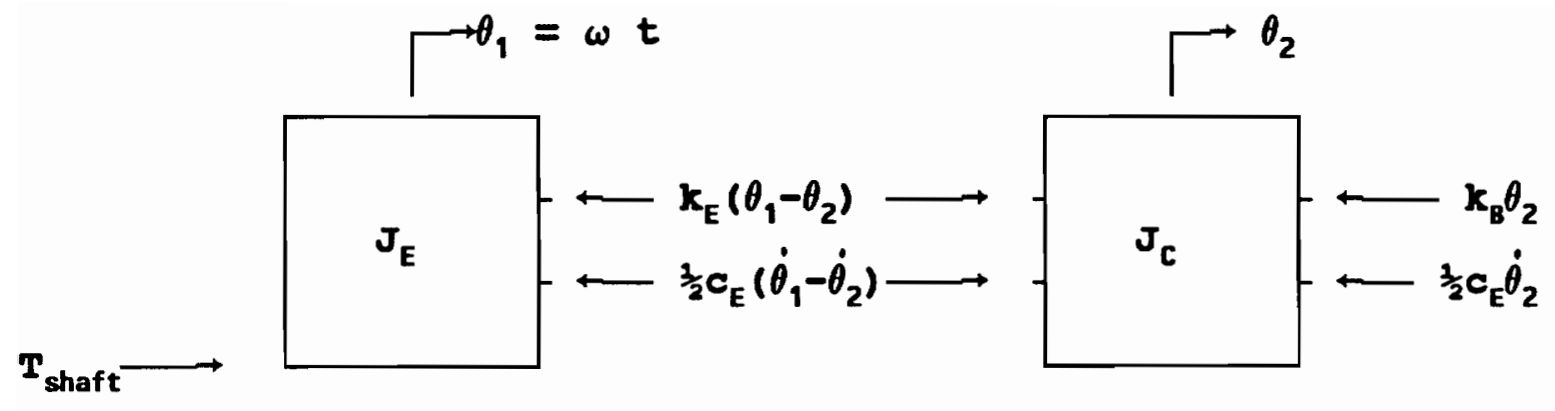

(a)

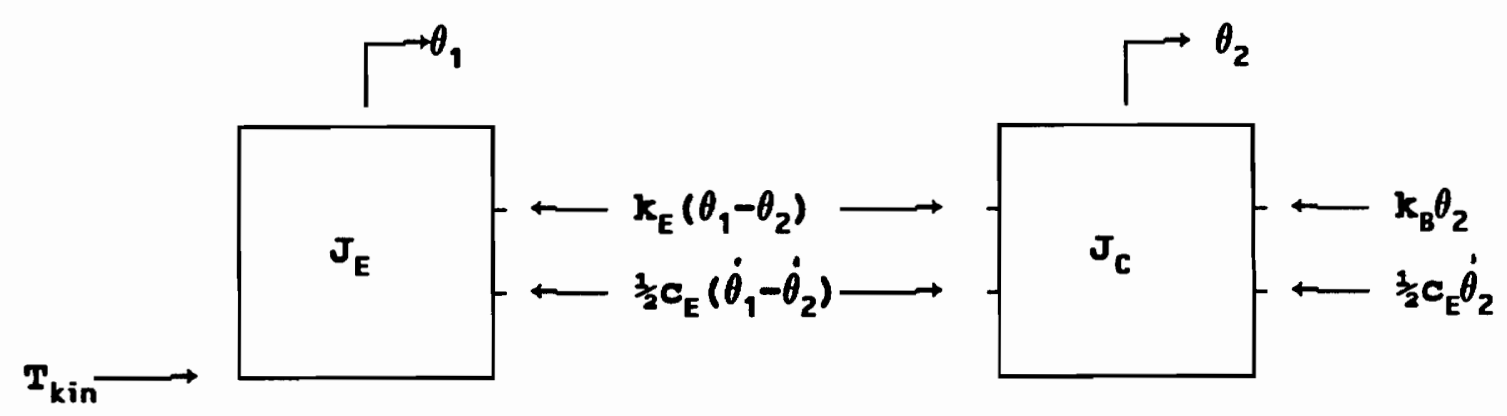

(b)

Figure 4.2 - Free body diagram of forces acting on system during (a) stick phase and (b) slip phase. 
conditions from each experiment. The forces acting on the system during elastomer slip is shown in Fig 4.2(b). The ordinary second order differential equations of motion are

$$
\begin{aligned}
& \ddot{\theta}_{1}-\frac{1}{J_{B}}\left[T_{k i n}-\frac{1}{2} C_{B}\left(\dot{\theta}_{1}-\dot{\theta}_{2}\right)-k_{B}\left(\theta_{1}-\theta_{2}\right)\right] \\
& \ddot{\theta}_{2}=\frac{1}{J_{C}}\left[C_{B}\left(\frac{1}{2} \dot{\theta}_{1}-\dot{\theta}_{2}\right)+k_{B}\left(\theta_{1}-\theta_{2}\right)-k_{B} \theta_{2}\right]
\end{aligned}
$$

The simulation continues using the final values of $\theta_{1}, \dot{\theta}_{1}, \theta_{2}$, and $\dot{\theta}_{2}$ during elastomer stick as initial conditions for elastomer slip. Once $\dot{\theta}_{1}=\omega$, the final values from the slip phase of the simulation are returned as initial conditions for equations (4.2) and (4.3) to complete the solution for the stick-slip cycle and resume the solution of the stick phase.

A computer program was written to solve the equations of motion and friction torque under the constraints described above. A fourth order fixed time step RungeKutta computational procedure [26] was used. The program requires input of system parameters $k_{B}, k_{E}, c_{E}$, angular velocity $\omega$, and levels of $T_{c r i t}$ and $T_{k i n}$. The time step chosen was 0.00002 seconds. The program listing is in Appendix F. 


\subsection{Theoretical Torsional Beam Stiffness}

It was necessary to determine the beam torsional stiffness, $k_{B}$, as a ratio of applied torque to angular displacement, $\theta_{2}$, of the clamps. This system parameter were used to compare the accuracy of $k_{B}$ calculated from the experimentally determined values of the elastomer spring rate, $k_{E}$, and the effective spring rate, $k_{E F F}$, of the system as per equation (3.1) in Section 3.3. Then values of $k_{B}$ for beam lengths of 9.2 and $12.3 \mathrm{~cm}$ were determined using experimental measures of $k_{E}$ and $k_{E F F}$ and used in the computer simulation of system response due to friction induced vibration.

Figure 4.3 illustrates the free body diagram of the beam system. The system is modelled as two cantilevered beams with end load $P$ and moment $M$ applied by an assumed rigid clamp with radius $R$. Coordinates $y_{B}$ and $\theta_{B}$, assumed to be at beam length $x=l$, along with their coordinate systems, are labeled in Figure 4.3. Also note angular rotations $\theta_{B}$ and $\theta_{2}$ are equivalent.

Beam displacements $y_{B}$ and $\theta_{B}$ are given by

$$
\begin{aligned}
& y_{B}=\frac{-P I^{3}}{3 E I}+\frac{M I^{2}}{2 E I} \\
& \theta_{B}=\frac{-P I^{2}}{2 E I}+\frac{M I}{E I}
\end{aligned}
$$

Substituting $-\theta_{B} R$ for $y_{B}$ in equation (4.6) gives equation (4.7), which are solved for $P$ and $M$.

$$
\begin{gathered}
-\theta_{B} R-\frac{-P I^{3}}{3 E I}+\frac{M I^{2}}{2 E I} \\
\theta_{B}-\frac{-P I^{2}}{2 E I}+\frac{M I}{E I}
\end{gathered}
$$




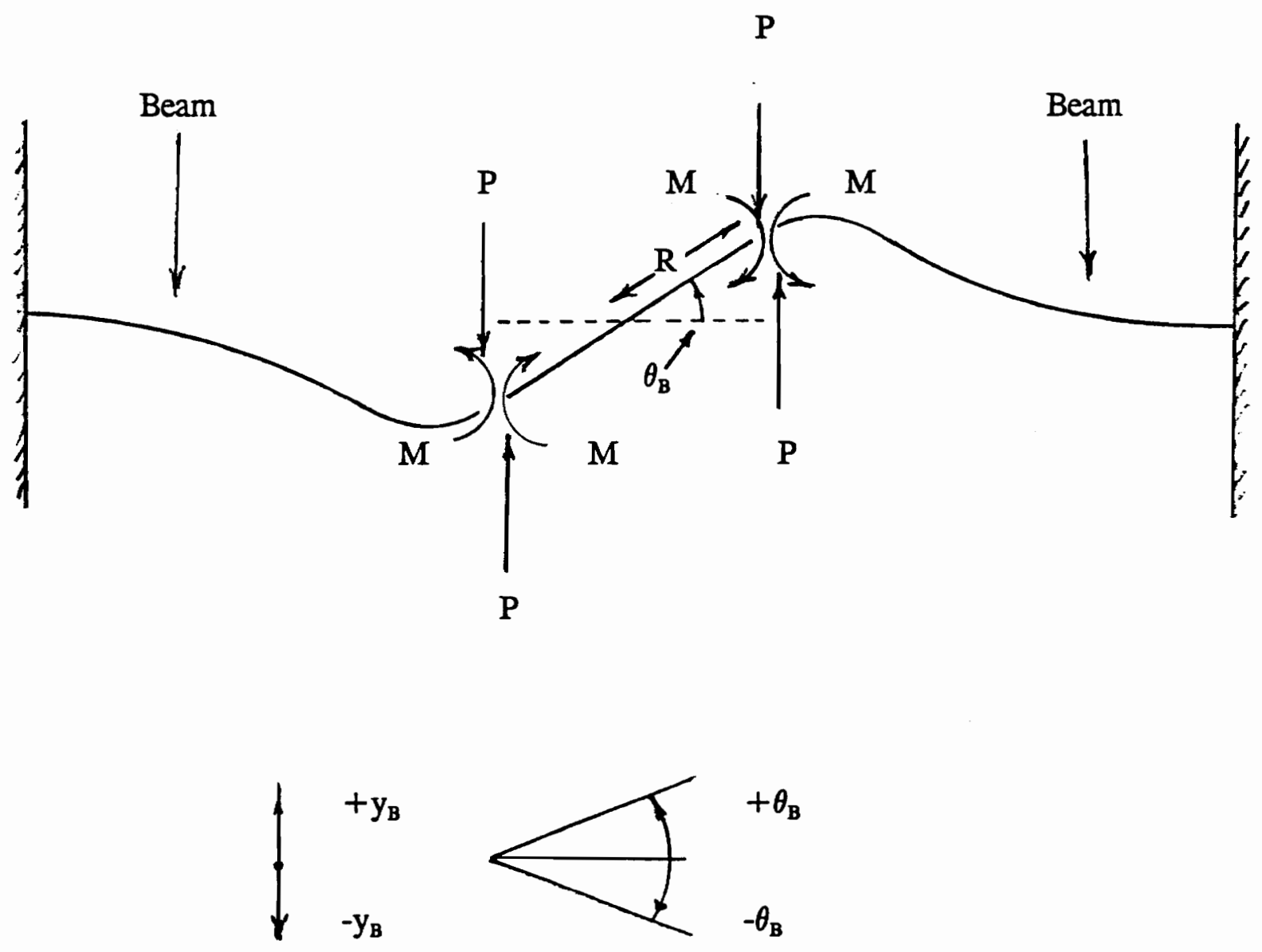

Figure 4.3 - Free body diagram of system to determine theoretical $k_{B}$. 


$$
\begin{gathered}
-R=-\left[\frac{P}{\theta_{B}}\right] \frac{I^{3}}{3 E I}+\left[\frac{M}{\theta_{B}}\right] \frac{I^{2}}{2 E I} \\
I=-\left[\frac{P}{\theta_{B}}\right] \frac{I^{2}}{2 E I}+\left[\frac{M}{\theta_{B}}\right] \frac{I}{E I} \\
P=\left[R+\frac{I}{2}\right] \frac{12 E I}{I^{3}} \theta_{B} \\
M=\left[R+\frac{2 I}{3}\right] \frac{6 E I}{I^{2}} \theta_{B}
\end{gathered}
$$

Solve for $k_{B}$ knowing

$$
k_{B}-\frac{T}{\theta_{B}}-\frac{2[P R+M]}{\theta_{B}}
$$

The torsional stiffness of the beams is determined by substitution of equation (4.9) into equation (4.10).

$$
k_{B}=\frac{24 E I}{I^{2}}\left[\frac{R^{2}}{1}+R+\frac{I}{3}\right]
$$

Using the following values for active beam lengths,

$$
\begin{aligned}
E & =2(10)^{11}[\mathrm{~Pa}] \\
I & =6.7746(10)^{-11}\left[\mathrm{~m}^{4}\right] \\
R & =0.0449[\mathrm{~m}] \\
I_{1} & =0.123[\mathrm{~m}] \\
I_{2} & =0.092[\mathrm{~m}]
\end{aligned}
$$

the theoretical torsional spring rates for the beams are found to be

$$
\begin{aligned}
& k_{B 1}=2214\left[\frac{\mathrm{Nm}}{\mathrm{rad}}\right] \\
& k_{B 2}=3745\left[\frac{\mathrm{Nm}}{\mathrm{rad}}\right]
\end{aligned}
$$

To ensure the assumptions of the model are correct, direct comparison of experimental friction behavior (presented in Section 5.0) and behavior obtain from the simulation in the time and frequency domains will be necessary. The simulation is 
important part of this thesis because it provides the solution of the instantaneous position, velocity, and acceleration of the system components. These variables cannot be measured experimentally. This data, viewed in the phase plane, will allow a complete assessment of the interaction of system dynamics with load and velocity which lead to self-excited, stick-slip oscillations. 


\subsection{Experimental Results}

\subsection{Regimes of Frictional Behavior}

These measurements were conducted to determine the type of frictional sliding that occurred for each material. The transition velocity, $V_{\text {critl }}$, was observed when frictional vibration changed from one type to another. Transition velocity $V_{\text {crit2 }}$, was found when frictional oscillations of any type ceased. The system parameters including beam stiffness and pressure were varied to determine any change in critical velocity bounding each regime. Changes in acoustic and system vibration were also noted for each frictional regime.

\subsubsection{Fluorocarbon Elastomer}

\section{Characterization of friction induced vibration}

The three frictional regimes observed were stick-slip, quasi-harmonic, and steady sliding friction behavior. Stick-slip oscillations were observed at the lowest velocities for each test case until a critical velocity was reached. Increasing the sliding speed past this first transition velocity either changed the waveform of the stick-slip oscillations or excited quasi-harmonic oscillations in the friction torque. The sliding speed was then increased further until friction induced vibration could not be sustained. At the largest interference fit between the elastomer and secondary shaft, the transition to steady sliding 
did not occur over the velocity range.

Stick-slip oscillations were characterized by the system torque which increased with time, and then suddenly dropped. The friction resembled the classic periodic sawtooth wave form. However, the rise in torque was not linear with respect to time. Figure 5.1(a) is a typical friction torque time trace for the fluorocarbon elastomer with infinite beam stiffness, $1 \mathrm{rad} / \mathrm{s}$ angular velocity, and $0.279 \mathrm{~mm}$ interference. When the support stiffness was changed to 2214 and $3745 \mathrm{Nm} / \mathrm{rad}$, frequencies higher than the stick-slip frequency were superimposed on the system torque during the stick phase. Figure 5.1(b) shows the torque time trace using the same velocity and load conditions and $3745 \mathrm{Nm} / \mathrm{rad}$ beam stiffness. At times the superimposed frequency (or frequencies) were much higher than the stick-slip frequency. This made the friction signal appear noisy and made it difficult to ascertain when slip occurred. For these cases the sudden, periodic change in beam acceleration amplitude indicated this phenomenon was stick-slip. Other oscillations were observed, but they did not have the traditional, sawtooth waveform. At times, the friction had a triangular waveform. The behavior shown in Figure 5.1(c) occurred at $k_{B}=2214 \mathrm{Nm} / \mathrm{rad}, \delta=0.178 \mathrm{~mm}$, and $4.3 \mathrm{rad} / \mathrm{s}$. This behavior is not stick-slip the relative velocity is not zero. This was found to be true because the rate of torque increase was much less than the rate of torque increase calculated using the shaft angular velocity, $\omega$, and the effective stiffness of the elastomer and beams, $k_{E F F}$ (see equation 5.1).

$$
\frac{T}{s}\left[\frac{N m}{s}\right]-k_{E F F}\left[\frac{N m}{r a d}\right] \omega\left[\frac{r a d}{s}\right]
$$



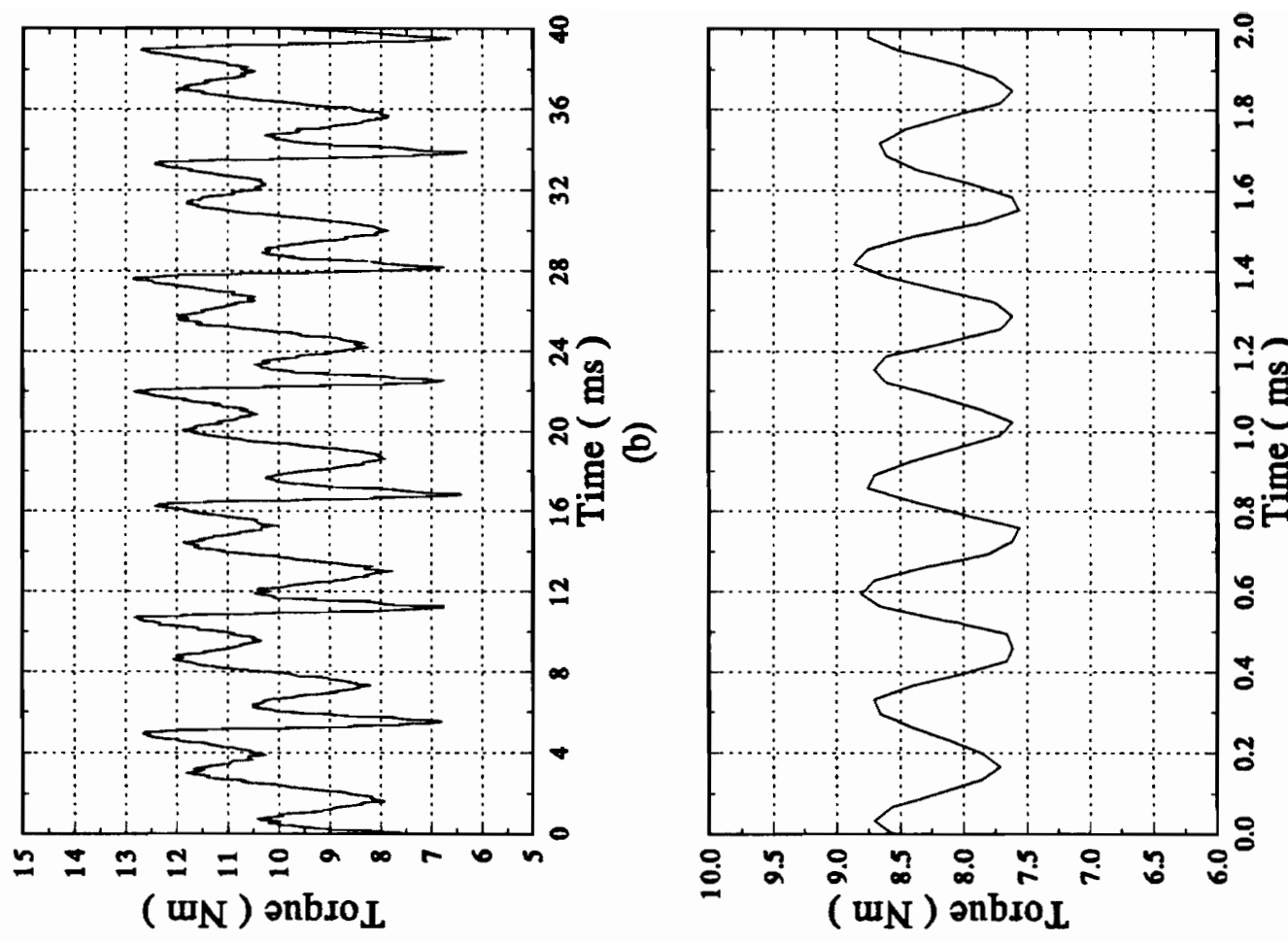

岕

2
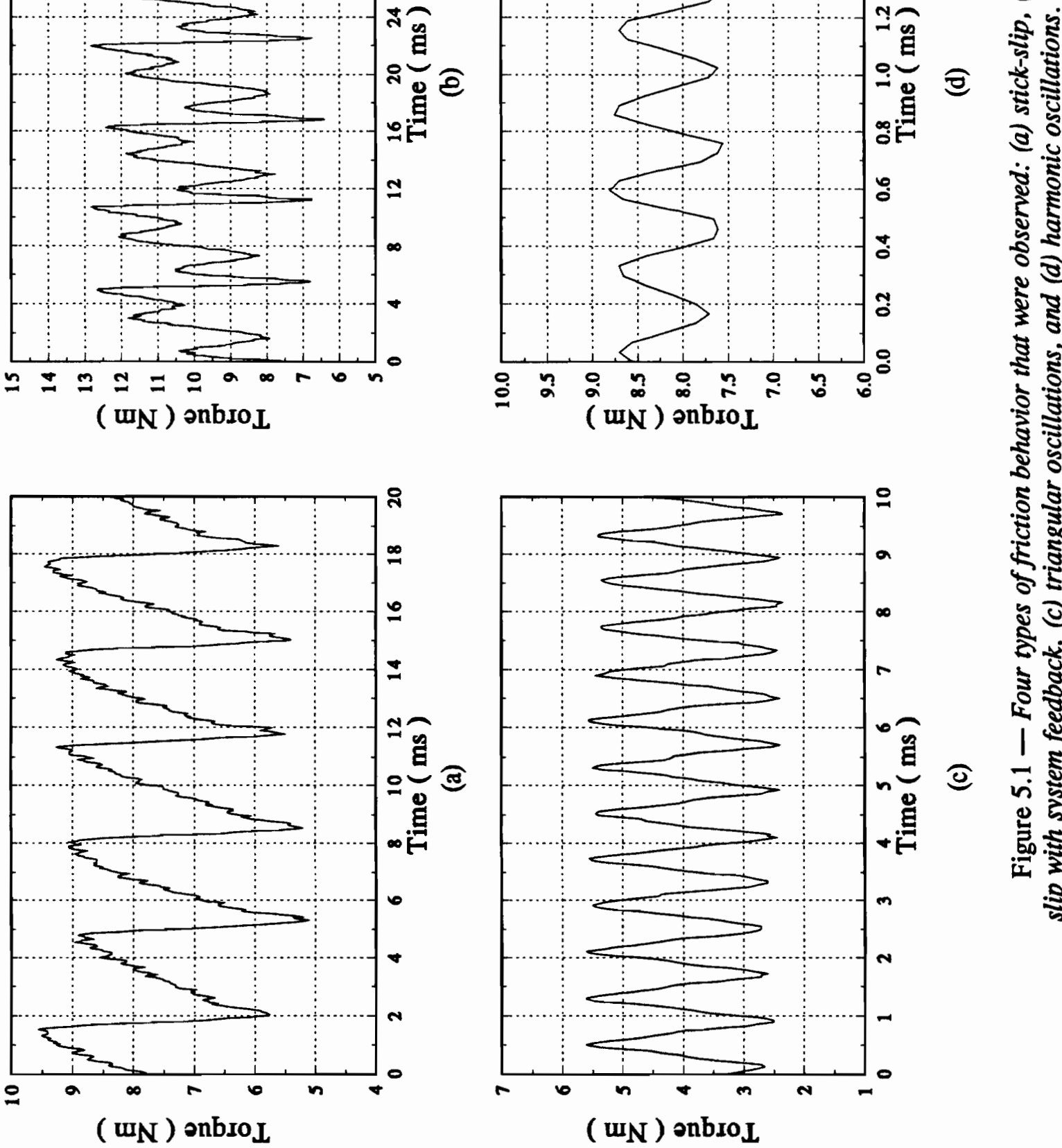

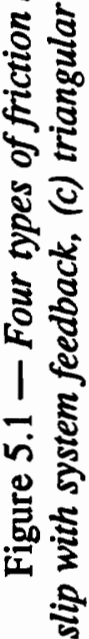

Experimental Results 
Quasi-harmonic oscillations were also observed. When this type of behavior was observed, the beams also vibrated harmonically. A typical friction signal for this type of behavior is shown in Figure 5.1(d). The operating conditions were $k_{B}=3745$ $\mathrm{Nm} / \mathrm{rad}, \delta=0.279 \mathrm{~mm}$, and $7.9 \mathrm{rad} / \mathrm{s}$. The amplitude of frictional oscillation was generally less when this type of self-induced vibration occurred.

When the shaft rotated inside the bushing with sufficient velocity, steady sliding occurred after a few oscillations. The torque remained constant at the mean level of the preceding oscillation. The oscillation resumed towards the end of the shaft displacement. The frictional vibration was excited at the beginning and end of each test because the shaft was not given a pure step change in velocity. The shaft velocity during steady state friction was recorded as the transition velocity, $V_{\text {crir } 2}$. Steady sliding occurred throughout the test when the velocity was greater than $V_{\text {crir }}$ during the entire shaft displacement.

Tables 5.1 and 5.2 show the influence stiffness and pressure had on the transition velocities from one type of frictional oscillation to another. Three frictional regimes exist for each test excluding the case with the clamp fixed at $0.178 \mathrm{~mm}$ inch interference fit. Table 5.1 shows the maximum velocity, $V_{\text {crill }}$, at which stick-slip occurs for the three pressure and system stiffness conditions. The critical velocity trends with increasing system stiffness are similar for the two lesser pressure conditions. Table 5.2 reveals similar critical velocity trends for $V_{\text {criz }}$ when the regime of steady sliding begins. As pressure increased, faster sliding conditions were required to obtain steady sliding conditions. Due to the torque and velocity limitations of the apparatus, steady sliding behavior was not obtained for the $0.381 \mathrm{~mm}$ interference fit tests. 
TABLE 5.1. Transition velocities, $V_{\text {crill }}$, from stick-slip to quasiharmonic oscillations (fluorocarbon elastomer).

\begin{tabular}{||c||c|c|c||}
\hline \hline \multirow{2}{|c||}{$\begin{array}{c}\text { Beam Stiffness, } \\
\text { Nm/rad }\end{array}$} & \multicolumn{3}{c||}{ Interference Fit, mm } \\
\cline { 2 - 4 } & 0.178 & 0.279 & 0.381 \\
\hline \hline 2214 & $<1 \mathrm{rad} / \mathrm{s}^{\dagger}$ & $4.5 \mathrm{rad} / \mathrm{s}$ & $>13 \mathrm{rad} / \mathrm{s}^{\ddagger}$ \\
3745 & $<1 \mathrm{rad} / \mathrm{s}^{\dagger}$ & $2.6 \mathrm{rad} / \mathrm{s}$ & $>13 \mathrm{rad} / \mathrm{s}^{\ddagger}$ \\
$\infty$ & $<1 \mathrm{rad} / \mathrm{s}^{\dagger}$ & $2.5 \mathrm{rad} / \mathrm{s}$ & $>13 \mathrm{rad} / \mathrm{s}^{\ddagger}$ \\
\hline
\end{tabular}

${ }^{\dagger}$ Quasi-harmonic oscillation present at lowest velocity tested. $\ddagger_{\text {No }}$ transition from stick-slip occurred up to $13 \mathrm{rad} / \mathrm{s}$.

TABLE 5.2. Transition velocities, $V_{\text {criz }}$, from quasi-harmonic oscillations to steady sliding (fluorocarbon elastomer).

\begin{tabular}{||c||c|c|c||}
\hline \hline \multirow{2}{||c||}{$\begin{array}{c}\text { Beam Stiffness, } \\
\text { Nm/rad }\end{array}$} & \multicolumn{3}{c||}{ Interference Fit, mm } \\
\cline { 2 - 4 } & 0.178 & 0.279 & 0.381 \\
\hline \hline 2214 & $4.6 \mathrm{rad} / \mathrm{s}$ & $7.9 \mathrm{rad} / \mathrm{s}$ & $\ldots{ }^{\dagger}$ \\
3745 & $5.9 \mathrm{rad} / \mathrm{s}$ & $12.5 \mathrm{rad} / \mathrm{s}$ & $\ldots{ }^{\dagger}$ \\
$\infty$ & $2.7 \mathrm{rad} / \mathrm{s}$ & $8.3 \mathrm{rad} / \mathrm{s}$ & $\ldots{ }^{\dagger}$ \\
\hline
\end{tabular}

${ }^{\dagger}$ Oscillations observed through $13 \mathrm{rad} / \mathrm{s}$. 


\section{Characterization of the frequency spectra}

The frequency content of the three types of behavior observed is distinct. The autospectra in Figure 5.2(a)-(d) corresponding to the time histories shown in Figure 5.1(a)-(d) illustrate the differences. The autospectrum of classical stick-slip in Figure 5.2(a) contains the peaks at 620 and $930 \mathrm{~Hz}$ which are harmonics of the slip frequency. The autospectrum in Figure 5.2(b) also shows the Fourier transform result of the nonharmonic slip event. In addition, the amplitude of the third harmonic is larger than expected due to the superimposed harmonic frequency component during the stick-phase. Figures 5.2(c) and (d) verify that the triangular and quasi-harmonic behavior consists primarily of a single frequency.

\section{Friction/velocity relationship}

The mean value of the torque oscillation was determined to find the torque/velocity relationship. This value was used because it was observed that the friction during stable sliding was the same as the mean value of the oscillation. Figure 5.3 shows the friction/velocity relationship of the fluoroelastomer at $\delta=0.179,0.278$, and $0.381 \mathrm{~mm}$. There is little variation in torque over an order of magnitude velocity increase. Although the support stiffness affected the velocities bounding each friction regime, ie., $V_{\text {critl }}$ and $V_{\text {criz } 2}$, it had little effect on the mean value of friction. The load was the only factor influencing the magnitude of friction torque. 

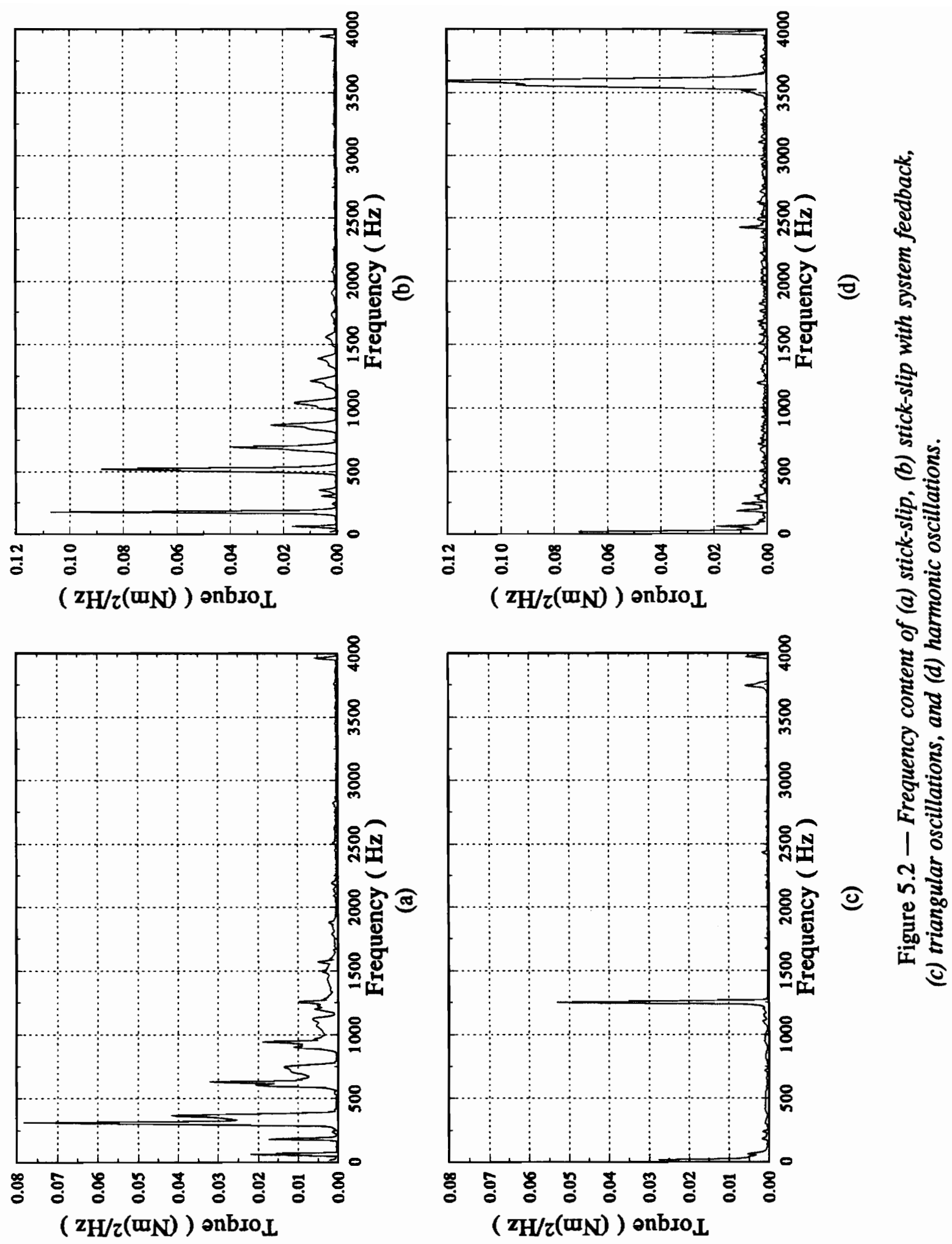


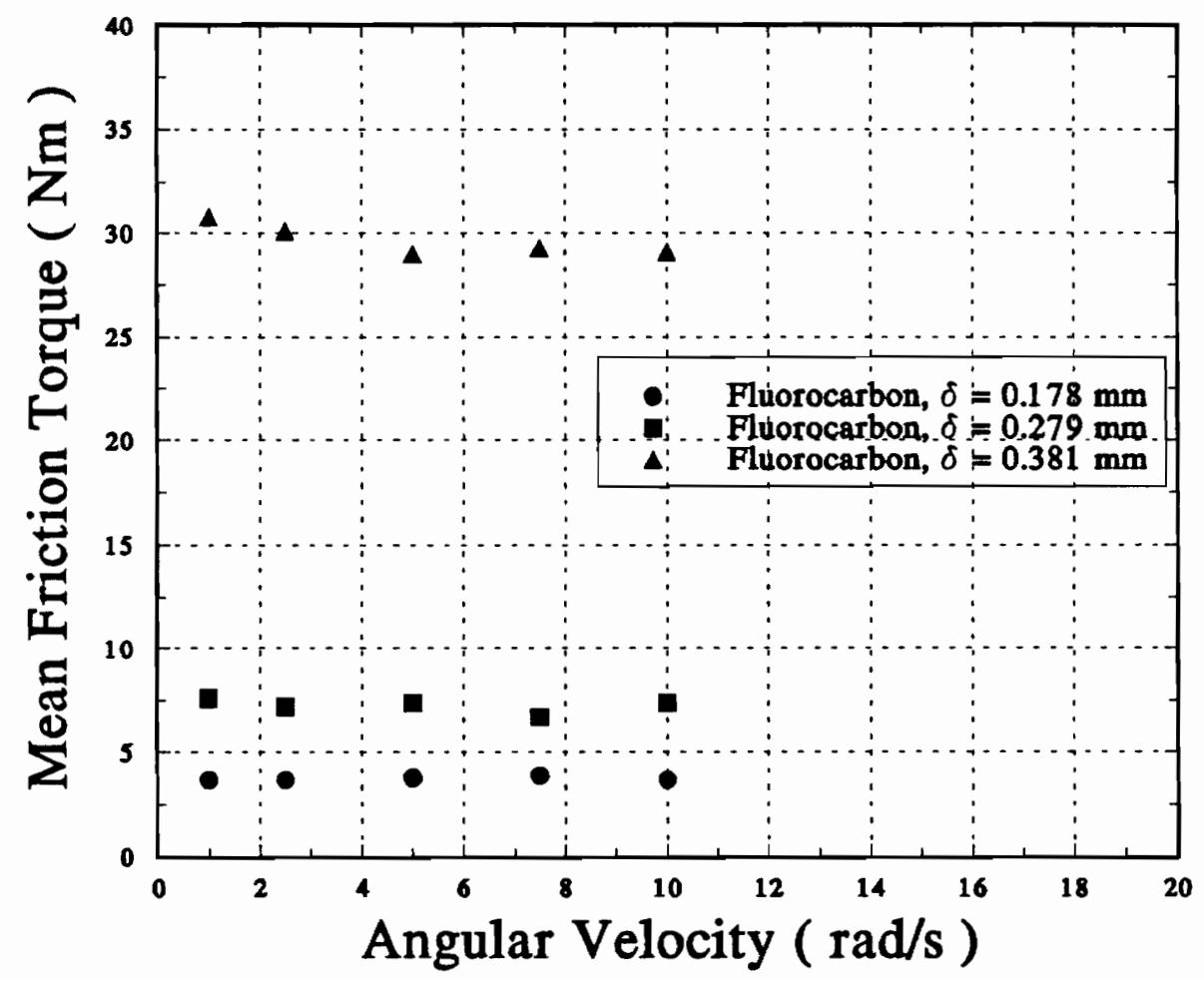

Figure 5.3 - Fluorocarbon friction torque/velocity relationships with $k_{B}=\infty$ (a) $\delta=0.178 \mathrm{~mm}$, (b) $\delta=0.279 \mathrm{~mm}$. and (c) $\delta=.381 \mathrm{~mm}$. 


\subsubsection{PDMS}

\section{Characterization of friction}

The friction behavior of the PDMS bushing was markedly different than that of the fluoro-elastomer. Vibration was not induced throughout the $0.25-17 \mathrm{rad} / \mathrm{s}$ velocity range. The steady sliding behavior is confirmed by an absence of oscillations in the beam acceleration time signal. A typical time history at $0.25 \mathrm{rad} / \mathrm{s}$, with $\delta=0.381 \mathrm{~mm}$ and 2,214 Nm/rad support stiffness, is shown in Fig 5.4. The friction maintains the steady sliding condition during the entire shaft displacement regardless of the interference fit or support stiffness.

\section{Friction/velocity relationship}

The friction/velocity relationship is also different for each material. The fluoroelastomer maintained nearly constant friction torque levels as shaft velocity increased. The PDMS experiments revealed an increasing torque trend with increasing velocity. Figures 5.5 and 5.6 indicate the friction torque nearly triples over the test range. The experiments also reveal that the frictional behavior is not affected by the change in support stiffness or interference fit.

\subsubsection{Natural Rubber}

Defining regimes of friction behavior was difficult during the Natural rubber investigation because of transient friction behavior. Periodic and aperiodic stick-slip occurred first. Figure 5.7 shows periodic stick slip and its autospectral density. The 


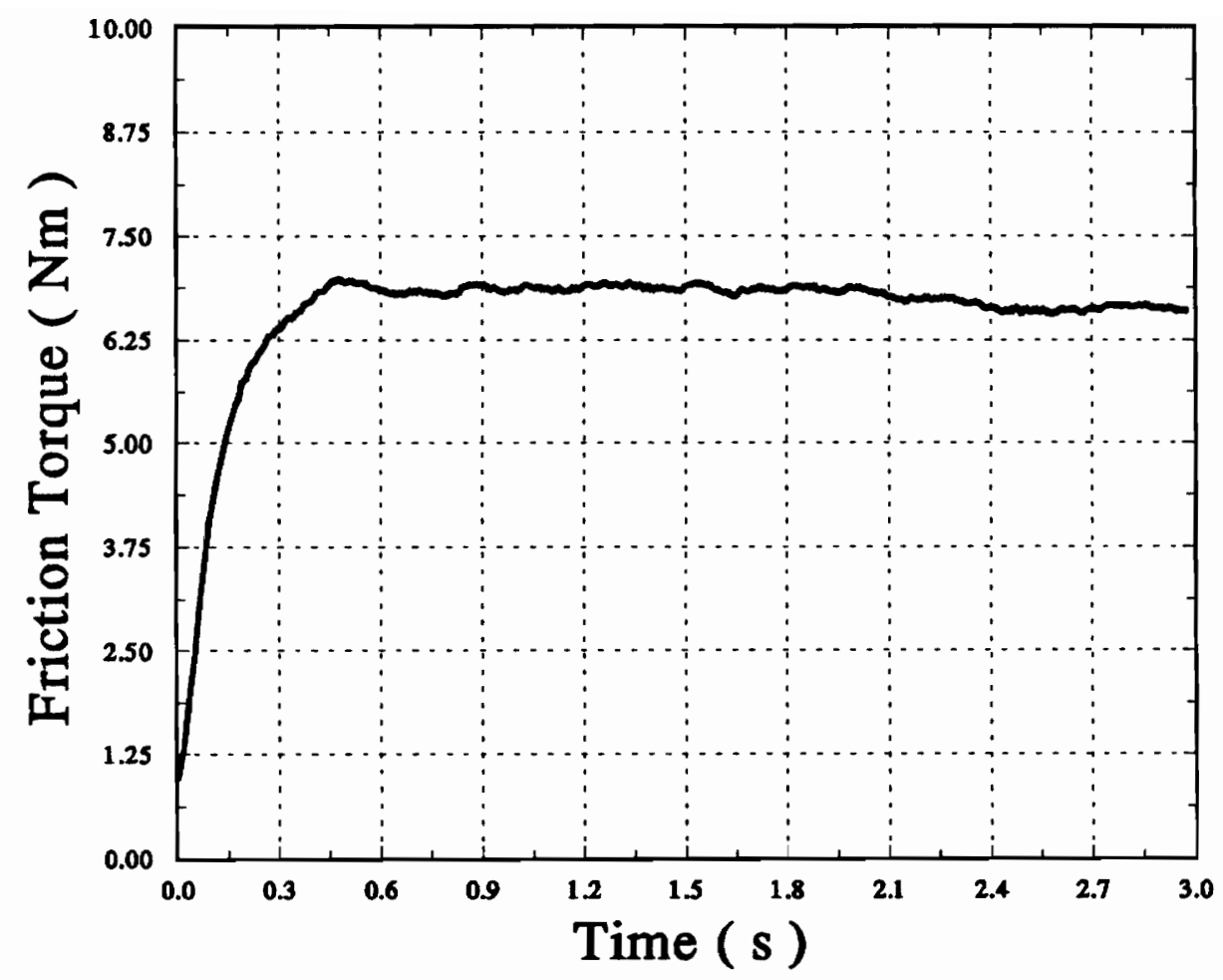

Figure 5.4 - Stable sliding behavior of PDMS with $\omega=0.25 \mathrm{rad} / \mathrm{s}$, $k_{B}=2,214 \mathrm{Nm} / \mathrm{rad}$, and $\delta=0.381 \mathrm{~mm}$. 


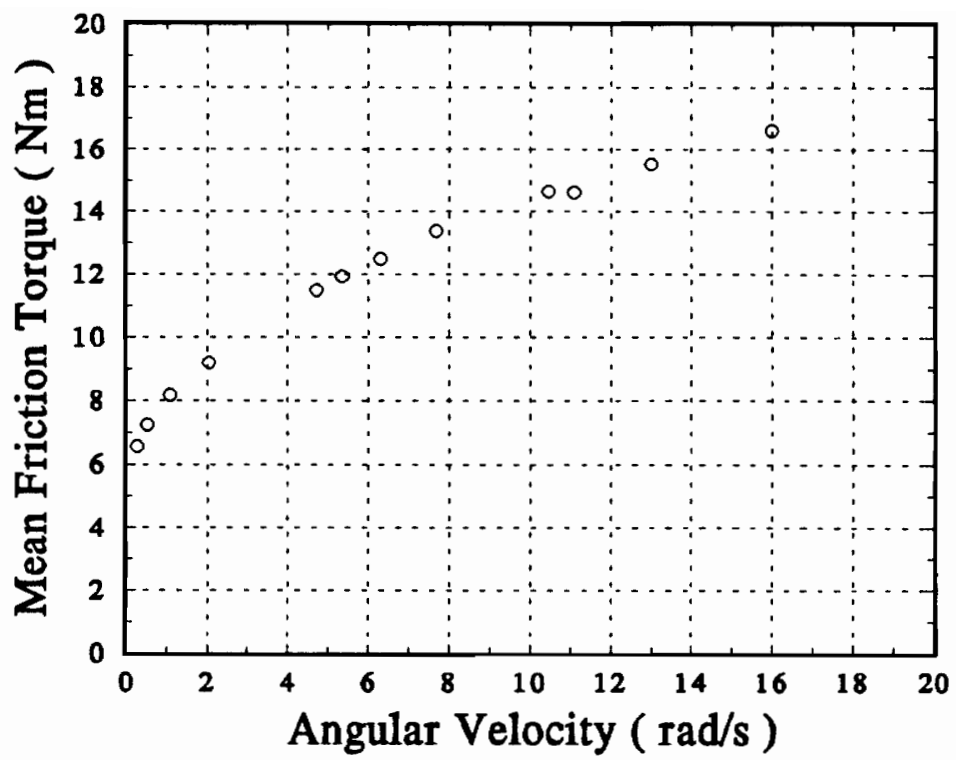

(a)

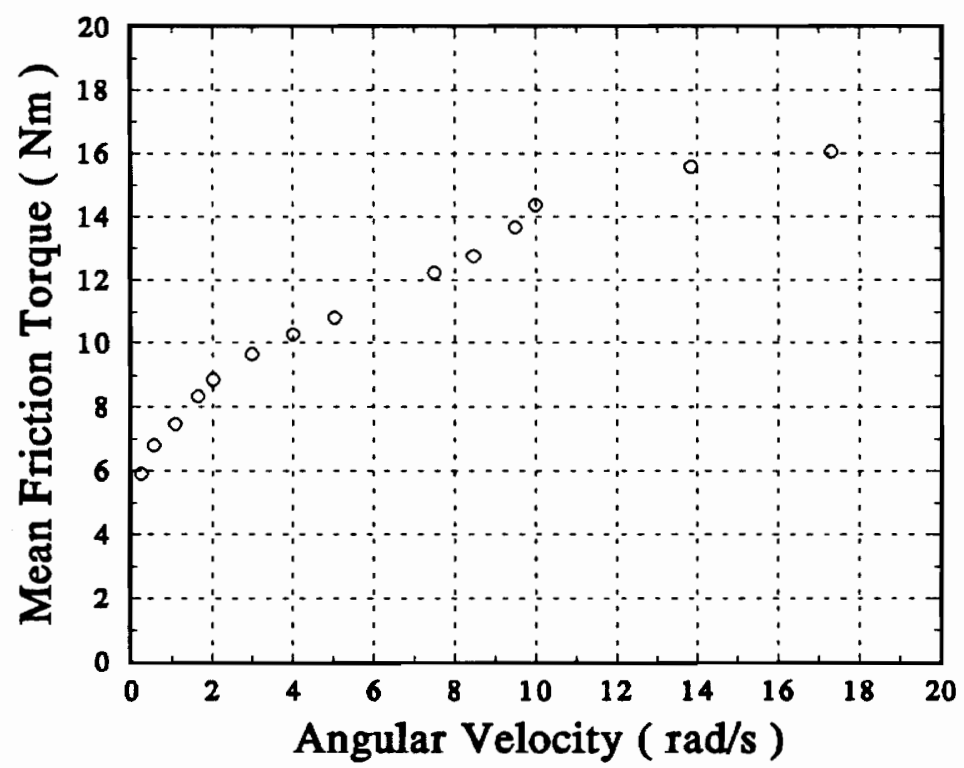

(b)

Figure 5.5 - PDMS friction torque/velocity relationships with (a) $k_{B}=2,214 \mathrm{Nm} / \mathrm{rad}, \delta=0.254 \mathrm{~mm}$ and (b) $k_{B}=\infty, \delta=0.254 \mathrm{~mm}$. 


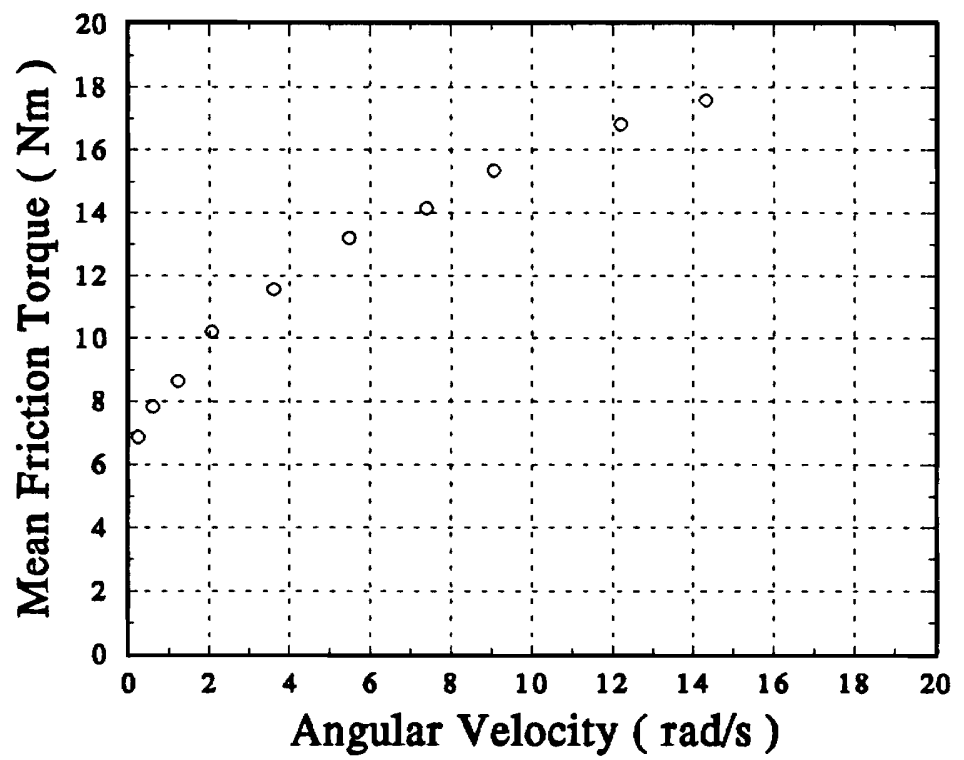

(a)

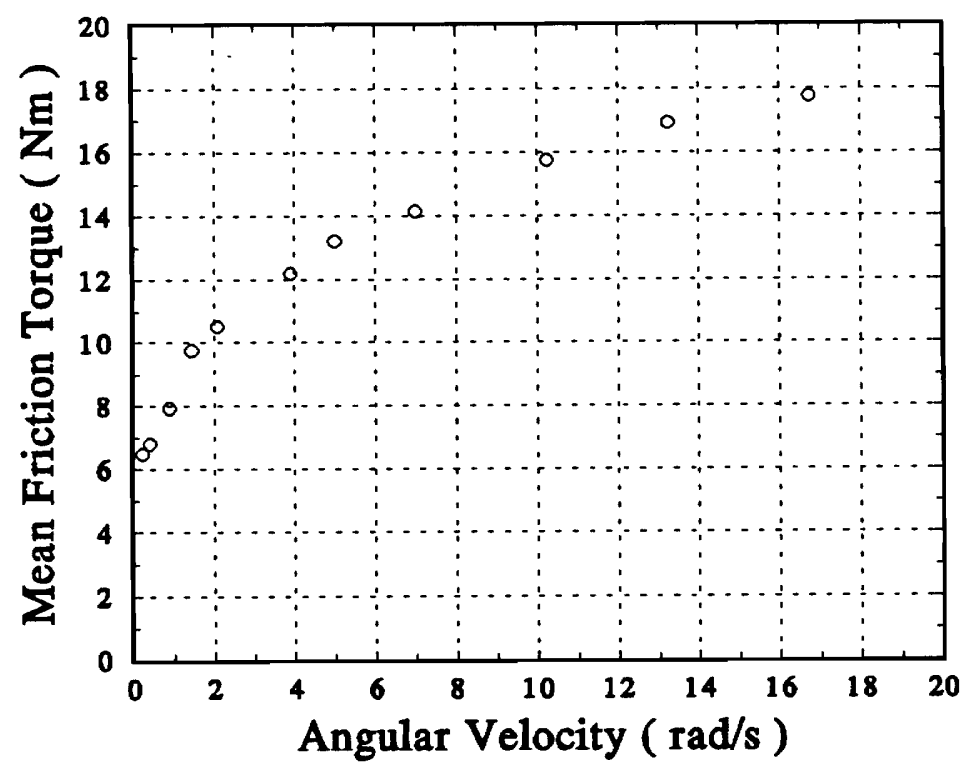

(b)

Figure 5.6-PDMS friction torque/velocity relationships with (a) $k_{B}=2,214 \mathrm{Nm} / \mathrm{rad}, \delta=0.381 \mathrm{~mm}$ and (b) $k_{B}=\infty, \delta=0.381 \mathrm{~mm}$. 


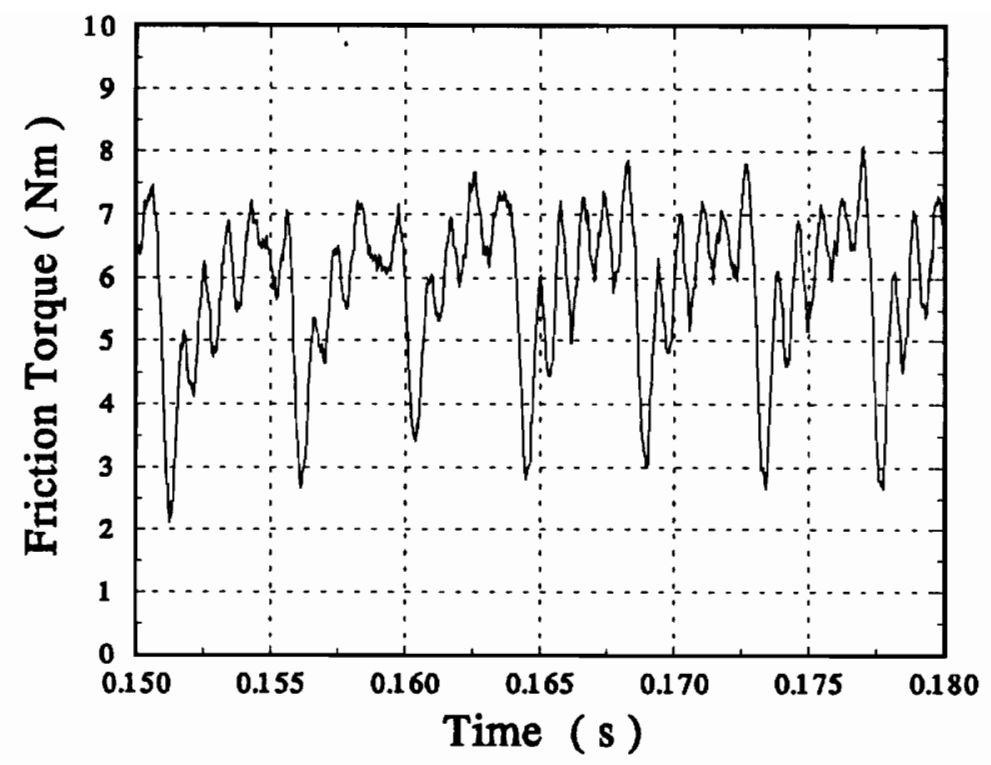

(a)

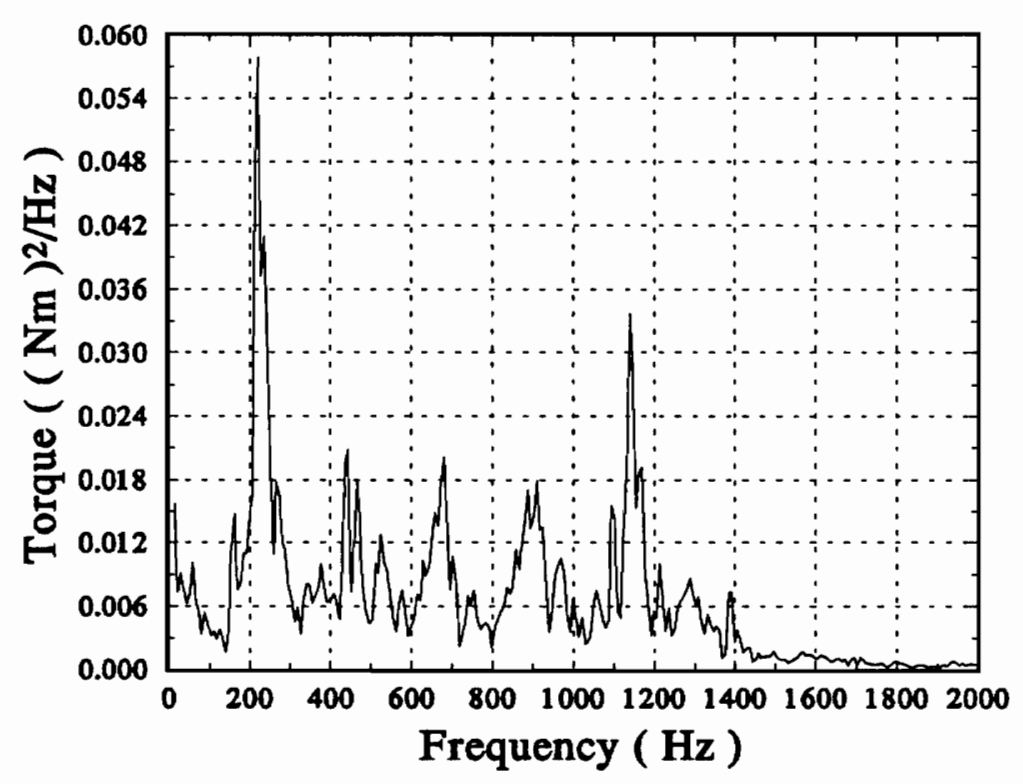

(b)

Figure 5.7 - Natural rubber friction behavior $k_{B}=2,214 \mathrm{Nm} / \mathrm{rad}, \delta=$ $0.127 \mathrm{~mm}$ ) (a) torque time history and (b) autospectral density. 
behavior was recorded at $\omega_{s}=10 \mathrm{rad} / \mathrm{s}$ and $\delta=0.127 \mathrm{~mm}$. The stick-slip behavior included a superimposed frequency during the stick phase. This behavior was noted for the fluorocarbon at $\omega_{s}=1 \mathrm{rad} / \mathrm{s}$ and the same support stiffness. The friction torque signal shows a loss of periodicity at $\omega_{s}=5 \mathrm{rad} / \mathrm{s}$ as seen in Figure 5.8. The autospectrum reveals a noisy band through $1250 \mathrm{~Hz}$.

After a few more shaft rotations a transformation in friction behavior was observed. Mean friction levels tripled and the vibration amplitude was low or not above the noise threshold of the friction torque signal. Figure 5.9 shows the friction and beam acceleration signals at $\omega_{s}=2.5 \mathrm{rad} / \mathrm{s}$. The acceleration signals reveal brief periods of beam excitation which quickly damp out.

The bushing/clamp assembly was removed a this behavior was observed. The shaft exhibited a transfer film of rubber. The transfer film was observable to the naked eye. Experiments were conducted with the transfer film at $\omega_{s}=1,2.5,5,7$, and 10 $\mathrm{rad} / \mathrm{s}$. $35 \mathrm{~mm}$ pictures of the shaft, taken with a photomacroscope, revealed most of the shaft was coated. Figure 5.10 shows the bare shaft with no rubber transfer. The waviness indicates the macro roughness of the shaft. Figure 5.11 shows the edge of the transfer film. The left half is the epoxy paint. The right half of the photograph is the transfer film. Figure 5.12 shows the transfer film at the center of the apparent contact region. Smearing and streaking is evident from these photographs. No friction/velocity relationships could be measured due to the inconsistent friction behavior.

Friction/velocity relationship

After removing the transfer film with acetone and isopropyl alcohol, experiments 


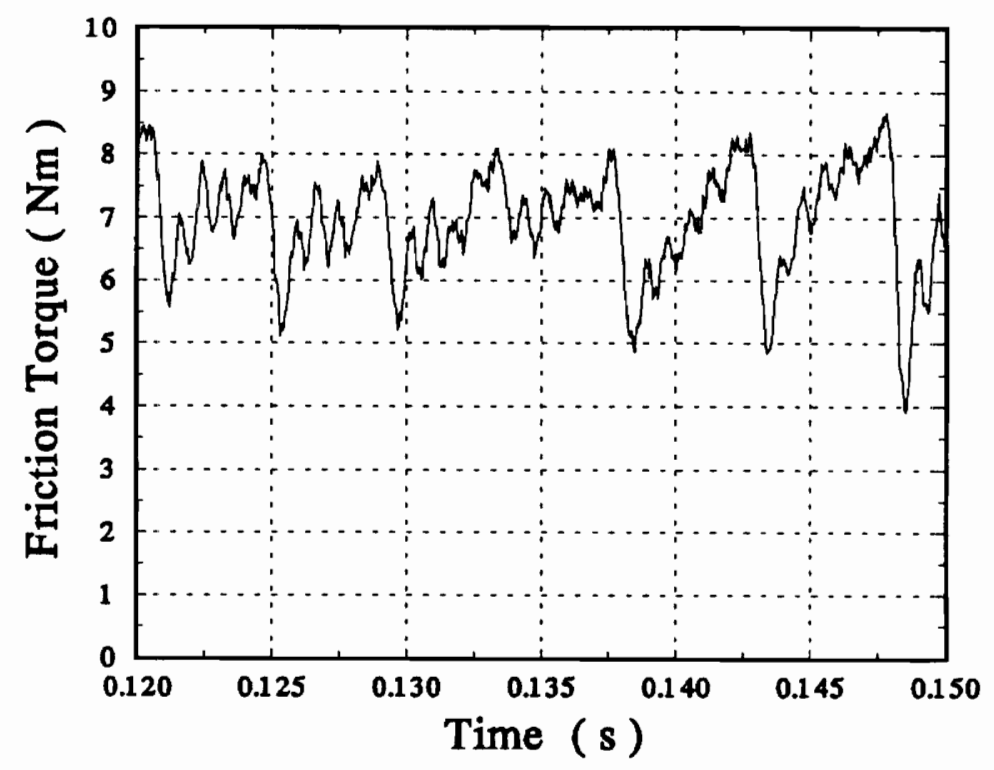

(a)

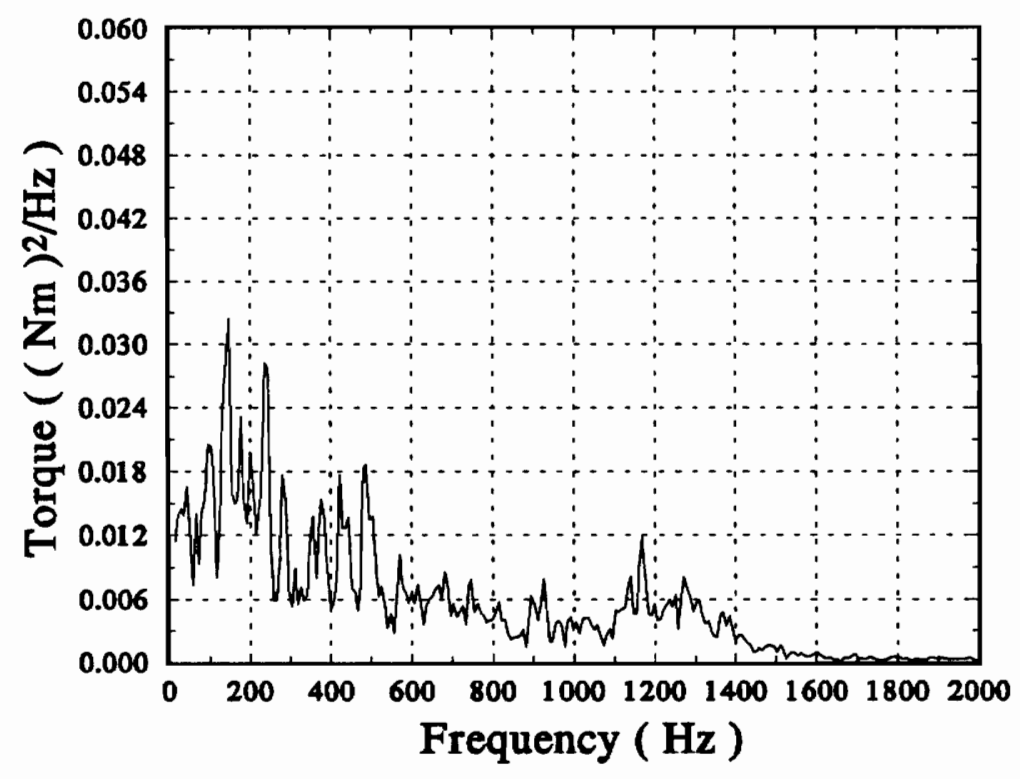

(b)

Figure 5.8 - Natural rubber friction behavior $k_{B}=2,214 \mathrm{Nm} / \mathrm{rad}, \delta=$ $0.127 \mathrm{~mm}$ ) (a) torque time history and (b) autospectral density. 


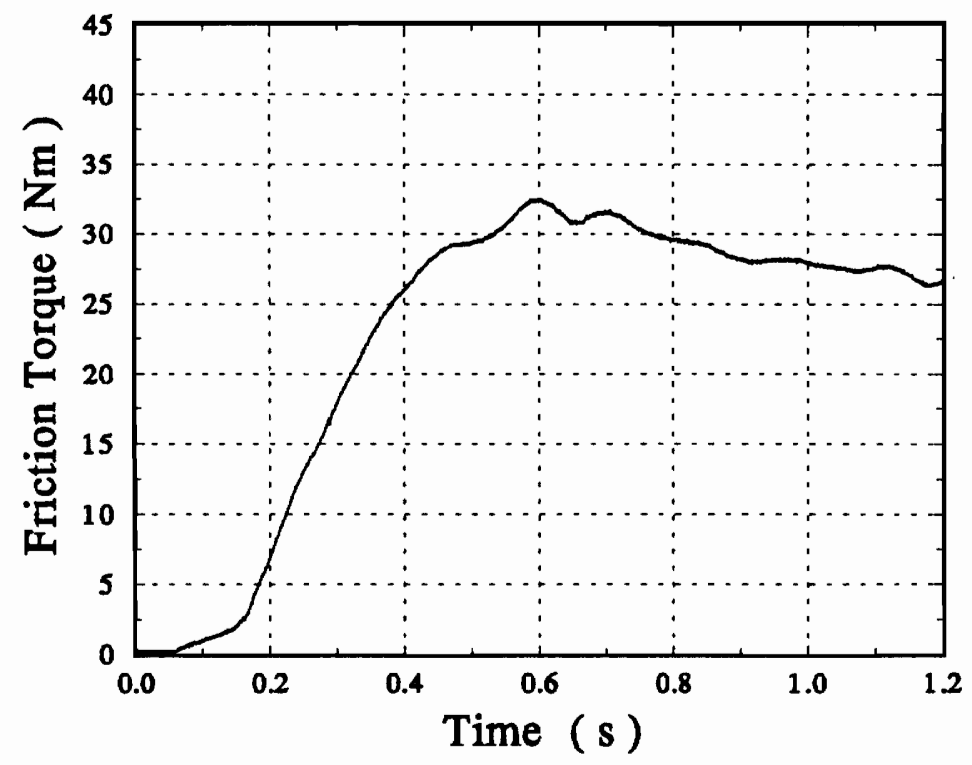

(a)

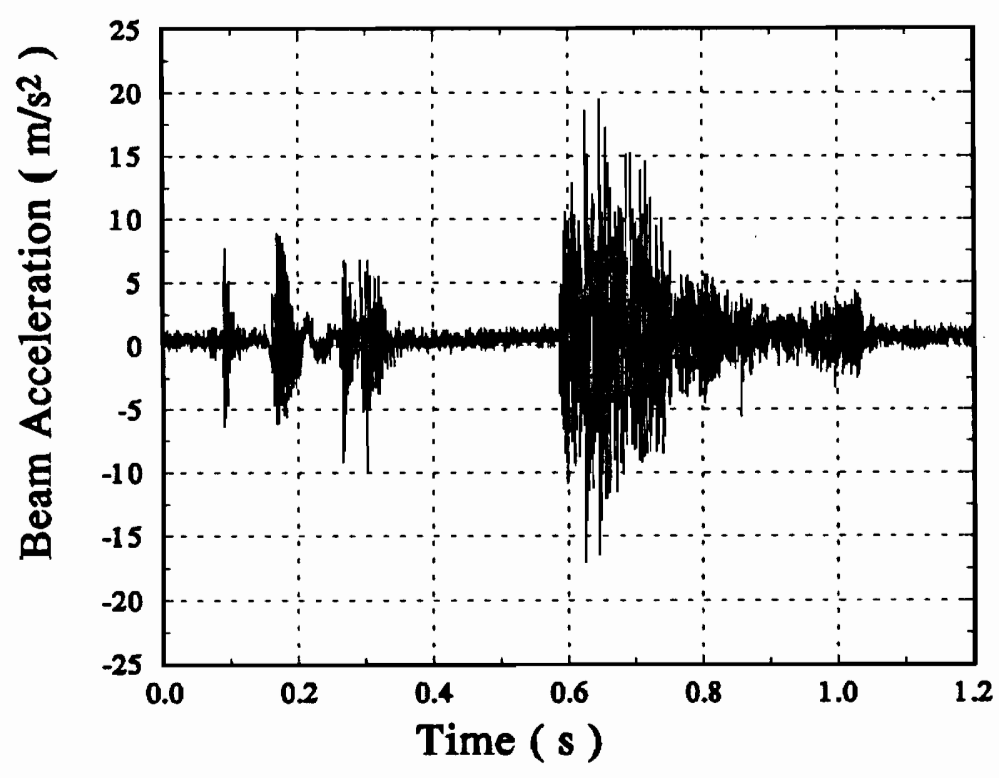

(b)

Figure 5.9-Natural rubber friction behavior $\left(k_{B}=2,214 \mathrm{Nm} / \mathrm{rad}, \delta=\right.$ $0.127 \mathrm{~mm}$ ) (a) torque time history and (b) autospectral density. 


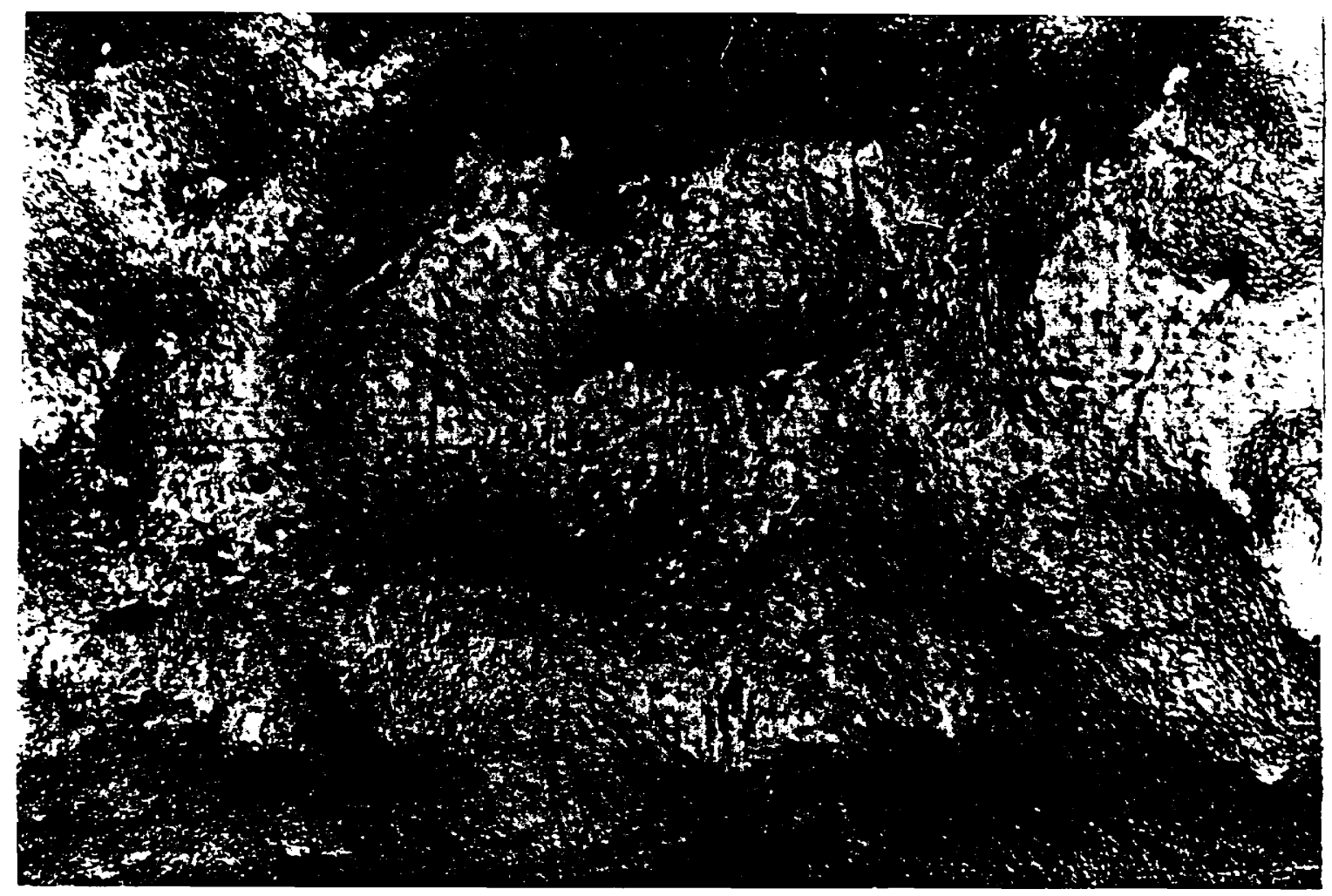

Figure 5.10 - Clean surface of epoxy painted shaft ( $25 x$ magnification ). 


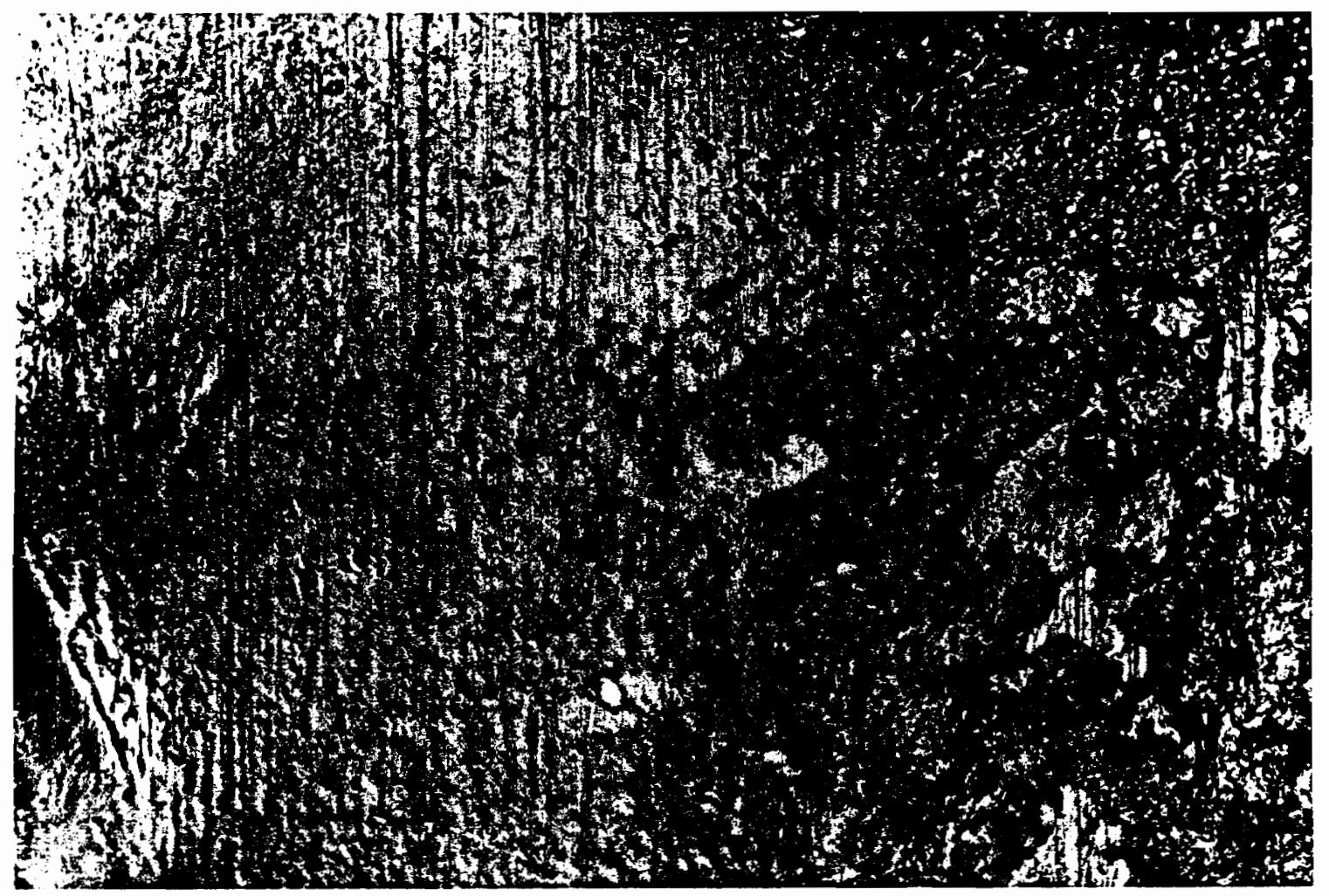

Figure 5.11 - Edge of natural rubber transfer film (25x magnification). 


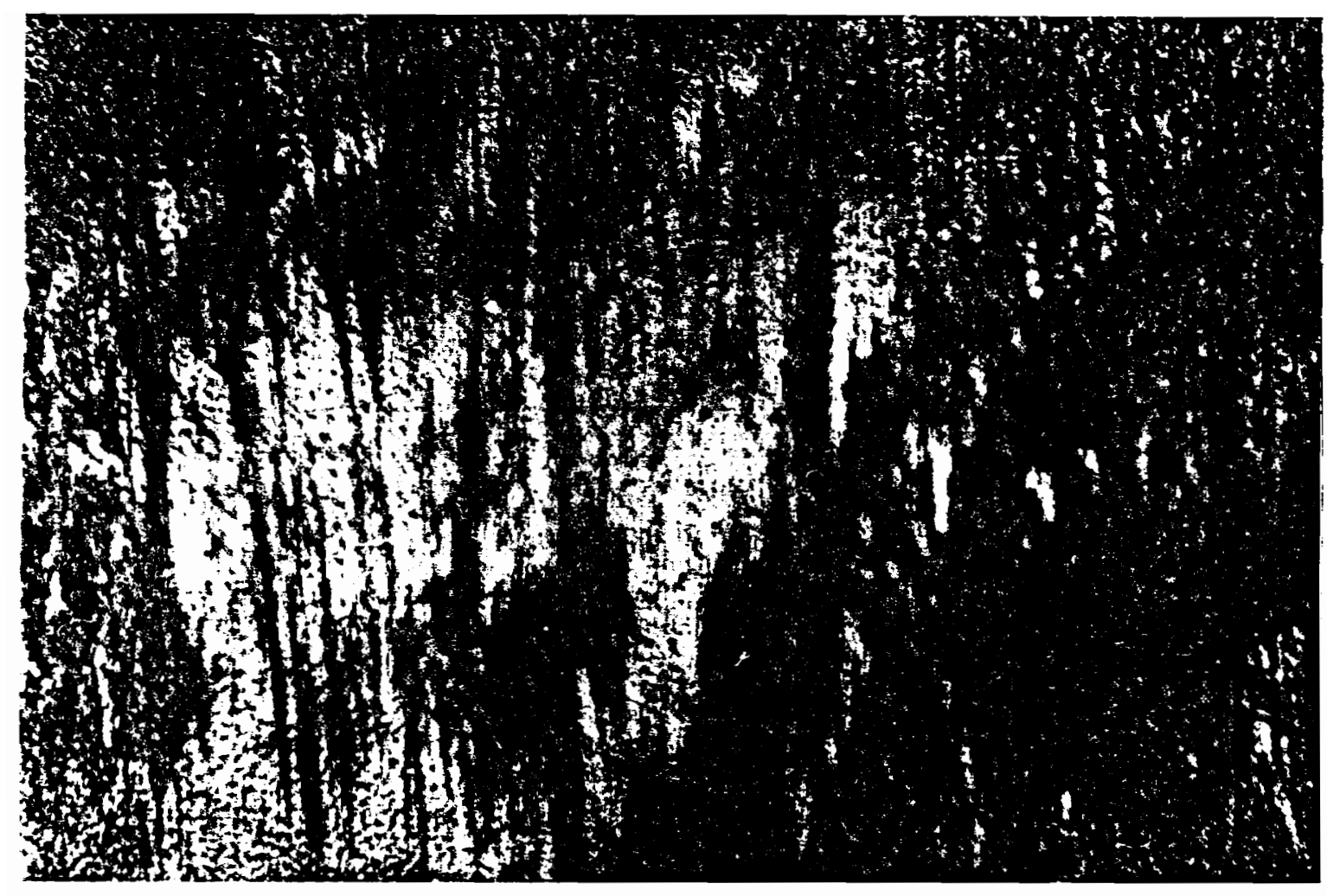

Figure 5.12 - Smeared natural rubber transfer film ( $25 x$ magnification ). 
at the same velocities were conducted to observe any behavioral changes in the friction torque. Pronounced friction induced vibration was observed through $19.75 \mathrm{rad} / \mathrm{s}$ for $k_{B}$ $=2,214 \mathrm{Nm} / \mathrm{rad}$ and through $10 \mathrm{rad} / \mathrm{s}$ for $k_{B}=3745 \mathrm{Nm} / \mathrm{rad}$. Stable sliding occurred at $\omega_{S}=1 \mathrm{rad} / \mathrm{s}$ and $k_{B}=2,214$. At higher shaft velocities signals were periodic while lower shaft velocities produced more noisy signals. The transfer film did not reform after the initial one was removed. The friction/velocity relationship shown in Figure 5.13 reveals the mean friction levels are constant throughout the range of velocity for the both support conditions. 


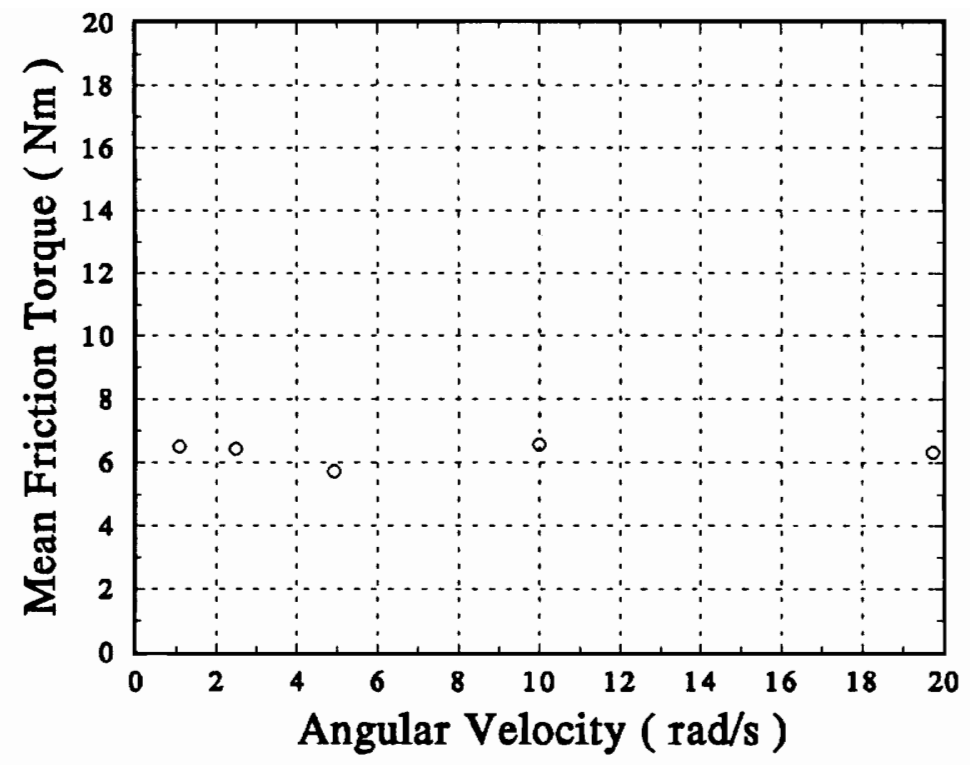

(a)

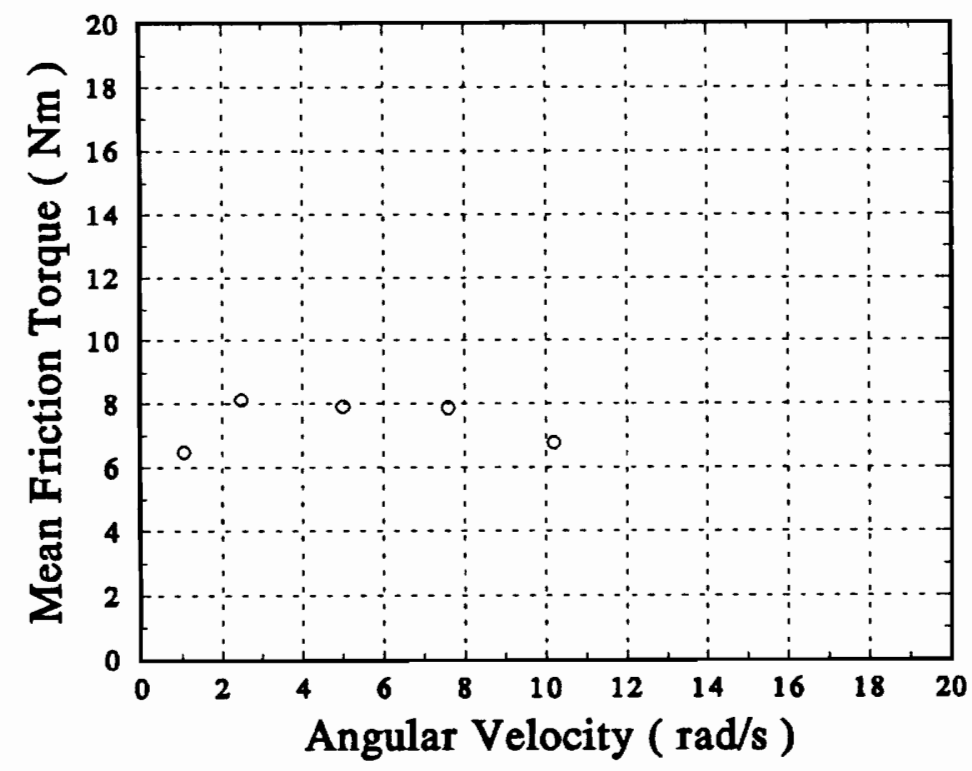

(b)

Figure 5.13 - Natural rubber friction/velocity relationships with (a) $k_{B}$ $=2,214 \mathrm{Nm} / \mathrm{rad}, \delta=0.127 \mathrm{~mm}$ and (b) $k_{B}=3,745 \mathrm{Nm} / \mathrm{rad}, \delta=0.127 \mathrm{~mm}$. 


\subsection{Vibration Study}

The fluorocarbon bushing was tested at five velocities for three different beam stiffnesses and three interference fits. Analyses of the torque time traces allowed comparison of mean friction levels and the amplitude of oscillation. The slope of the friction torque during the stick phase was compared to the product of the effective stiffness of the beams and elastomer and the angular velocity to determine when the oscillations were stick-slip. The autospectra of the torque and the acceleration signals were analyzed to compare the frequency components for each test condition. These analyses make it possible to determine how operating conditions including stiffness, velocity, and pressure affect friction character. Audible noise and system response were correlated with friction to determine how operating conditions promote noise generation.

\subsubsection{Friction Results-Fluorocarbon}

\section{Effect of velocity and interference fit}

At $0.178 \mathrm{~mm}$ bushing interference and $k_{B}=2,214 \mathrm{Nm} / \mathrm{rad}$, the amplitude of oscillation and dc level of the friction force did not vary with velocity from 1 to 2.5 $\mathrm{rad} / \mathrm{s}$. The friction induced vibrations in this velocity range were found to be stick-slip. The autospectra of the friction for these two velocities indicate the frequencies did not change with velocity.

Increasing the normal pressure $(0.279 \mathrm{~mm}$ interference) caused many changes in the friction torque. Slip amplitudes were greater as a result of higher interfacial 
pressure. The mean torque level was increased by a factor of two. Stick-slip frequencies were less than those at $0.178 \mathrm{~mm}$ interference fit. Figure 5.14 illustrates how frequencies increase with velocity until $5 \mathrm{rad} / \mathrm{s}$. The friction autospectra at 1 and 2.5 $\mathrm{rad} / \mathrm{s}$ show a fundamental peak corresponding to the slip frequency and higher energy at the third harmonic indicating the frequency of the superimposed oscillation at the third harmonic. At 5 and $7.5 \mathrm{rad} / \mathrm{s}$, the primary frequency is at the fourth harmonic of the slip frequency. Vibration feedback from the beam to the interface was indicated by dominant peaks in the autospectrum of the beam acceleration which were at the same frequency as shown in Figure 5.14. From $5 \mathrm{rad} / \mathrm{s}$ until the transition velocity for steady sliding, the frequency of the peaks in the autospectra remain constant.

Analysis of the friction signal at $0.381 \mathrm{~mm}$ interference fit reveals friction levels are several times greater than those for smaller interference fits. The slip amplitude is also much larger at this pressure. The autospectra indicates stick-slip frequency (and its associated harmonics) increases 10 to $25 \mathrm{~Hz}$ with each $2.5 \mathrm{rad} / \mathrm{s}$ velocity increment. At constant beam stiffness and velocity, the fundamental frequency of vibration was smaller than for lesser interference fits while the peak amplitude of the frequency components in the autospectrum increases. This effect, shown in Figure 5.15(a) and (b), is independent of support stiffness.

\section{Effect of system dynamics}

The beam stiffness was increased to $3,745 \mathrm{Nm} / \mathrm{rad}$ to observe changes in friction behavior. Increasing sliding speeds through $5 \mathrm{rad} / \mathrm{s}$ significantly affected the friction frequency content as the autospectra show in Figure 5.16(b). This effect was not 


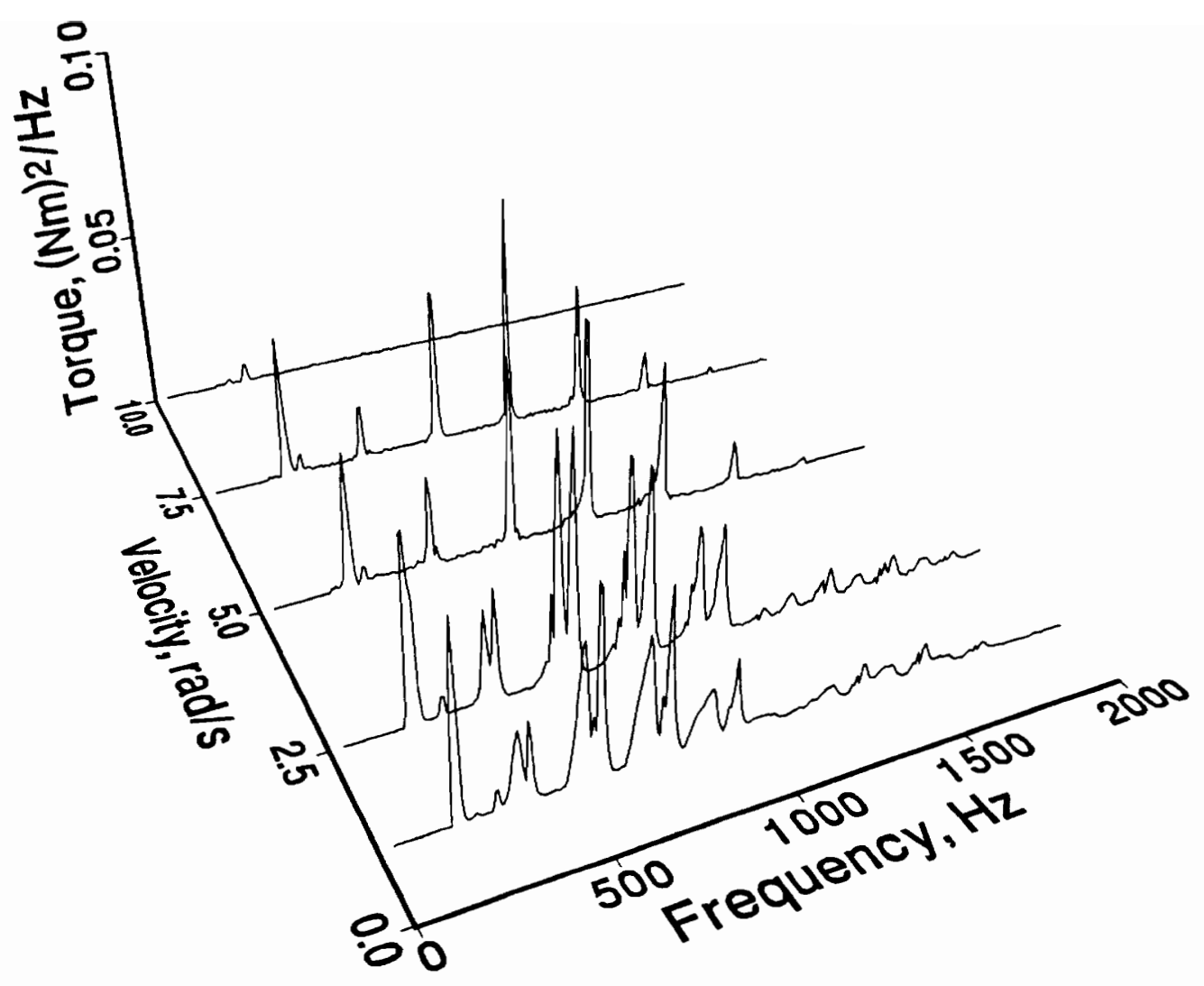

Figure $5.14-$ Frequency content of the friction torque with increasing velocity $\left(\delta=0.279 \mathrm{~mm}, k_{B}=2214 \mathrm{Nm} / \mathrm{rad}\right)$. 


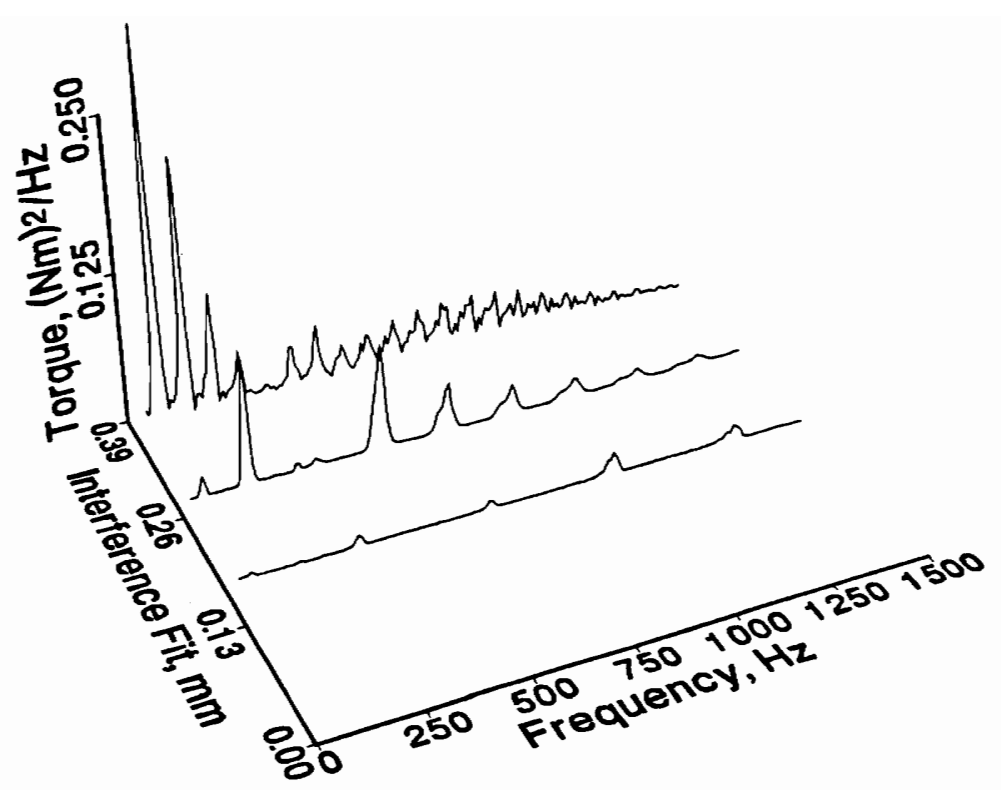

(a)

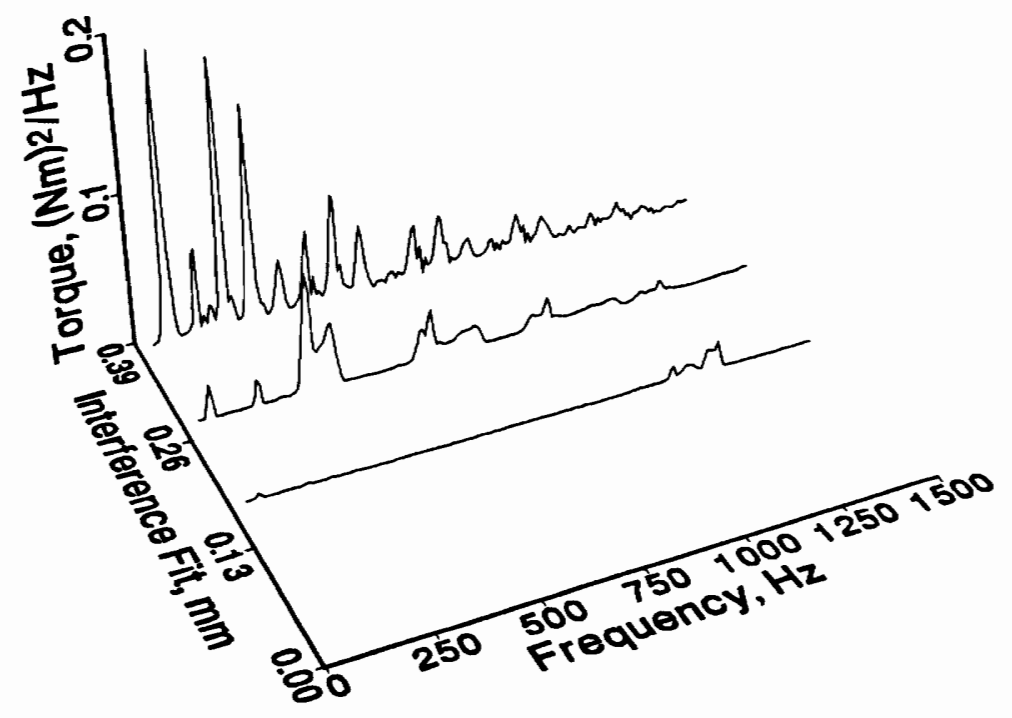

(b)

Figure 5.15 - Frequency content of the friction torque with increasing interference fit at $\omega=1 \mathrm{rad} / \mathrm{s}$ with (a) $k_{B}=2214 \mathrm{Nm} / \mathrm{rad}$ ) and (b) $k_{B}=\infty$.

Experimental Results

67 
observed at the $2,214 \mathrm{Nm} / \mathrm{rad}$ stiffness as seen in Figure 5.16(a).

When the interference fit was increased $(\delta=0.279 \mathrm{~mm})$, the peaks in the autospectrum remained constant from 2.5 to $7.5 \mathrm{rad} / \mathrm{s}$. The peaks were identified as the harmonics of the fundamental frequency, and the peak at associated with feedback from the beams. Thus increasing stiffness for this interference fit increased stick-slip frequency and increased the velocity range over which frequency content did not change.

When the clamp assembly was fixed to prevent vibration about the shaft and the interference was set at $0.178 \mathrm{~mm}$, the friction torque oscillated at a single frequency. The autospectra of these friction signals indicated peaks at $1,250 \mathrm{~Hz}$ over the velocity range where vibration occurred. However, at $\delta=0.279 \mathrm{~mm}$ and $0.381 \mathrm{~mm}$, a significant increase in frequency occurred throughout the velocity range. The friction autospectrum for the $\delta=0.279 \mathrm{~mm}$ fit in Figure 5.17 illustrates this frequency increase.

The frequency content changes considerably as support stiffness is increased while velocity and pressure remain constant. It is evident from Figure 5.18 that system stiffness affected the frequency content of the friction signal while velocity remained constant. Figure 5.18(a) contains the friction autospectra at $2.5 \mathrm{rad} / \mathrm{s}$ and $0.178 \mathrm{~mm}$ fit at each stiffness condition. Figure 5.18(b) illustrates the change in frequency content also occurred at $7.5 \mathrm{rad} / \mathrm{s}$ and $0.279 \mathrm{~mm}$ fit. The behavior is accentuated with larger interference fits because of longer stick time at higher normal pressures. 


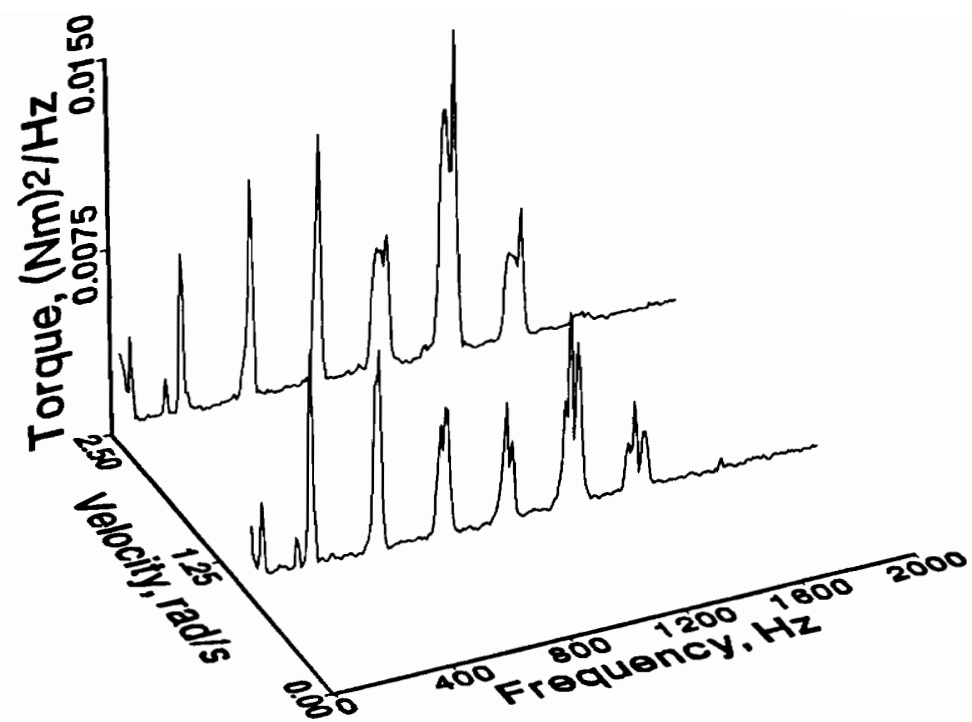

(a)

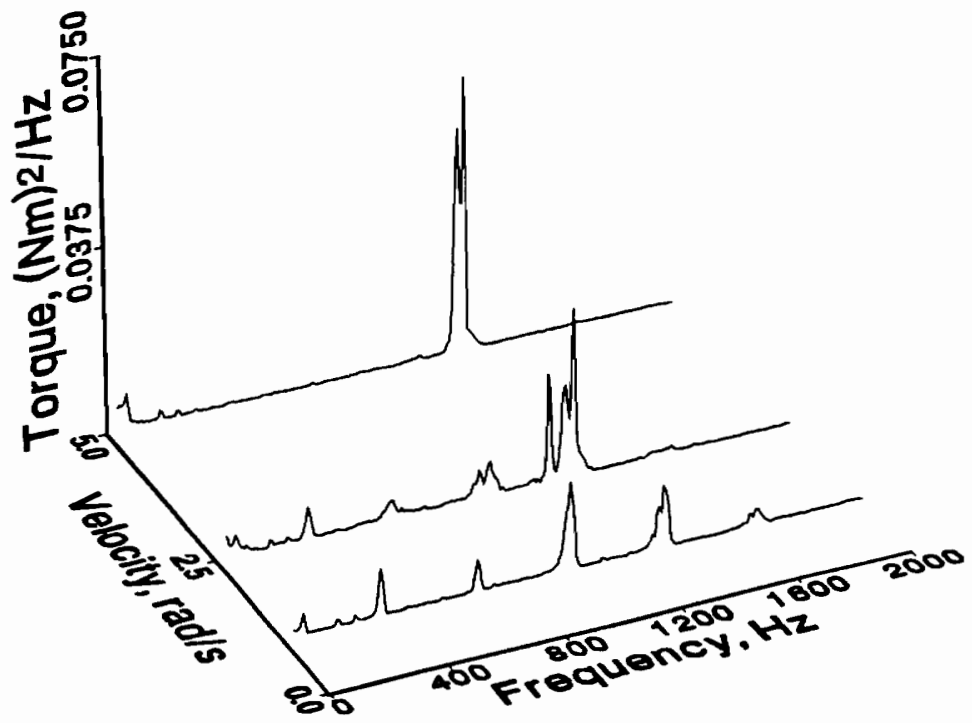

(b)

Figure 5.16 - Frequency content of the friction torque with increasing velocity $\left(\delta=0.178 \mathrm{~mm}\right.$ ) (a) $k_{B}=2,214 \mathrm{Nm} / \mathrm{rad}$ and (b) $k_{B}=$ infinite. 


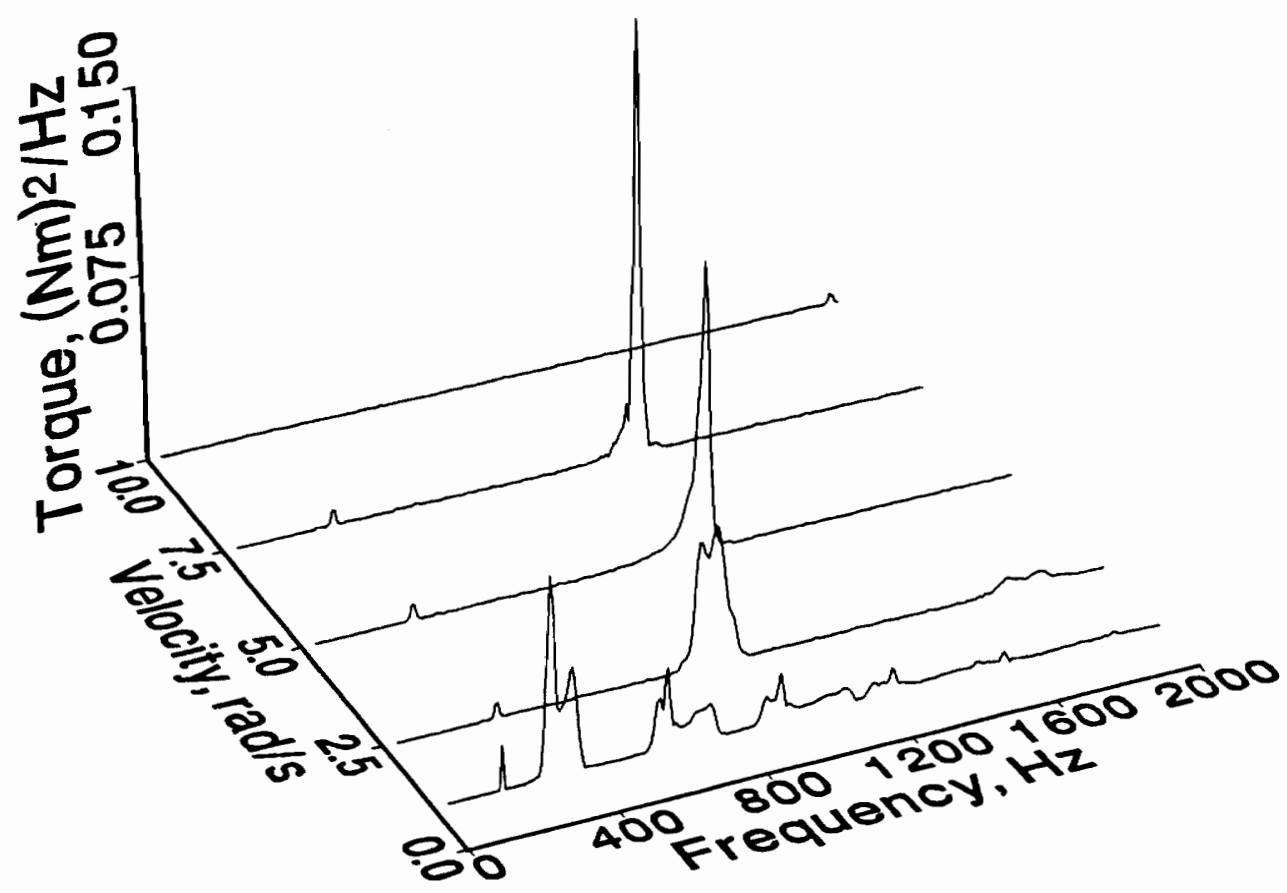

Figure 5.17 - Frequency content of the friction torque with increasing velocity $\left(\delta=0.279 \mathrm{~mm}, k_{B}=\infty\right)$.

Experimental Results

70 


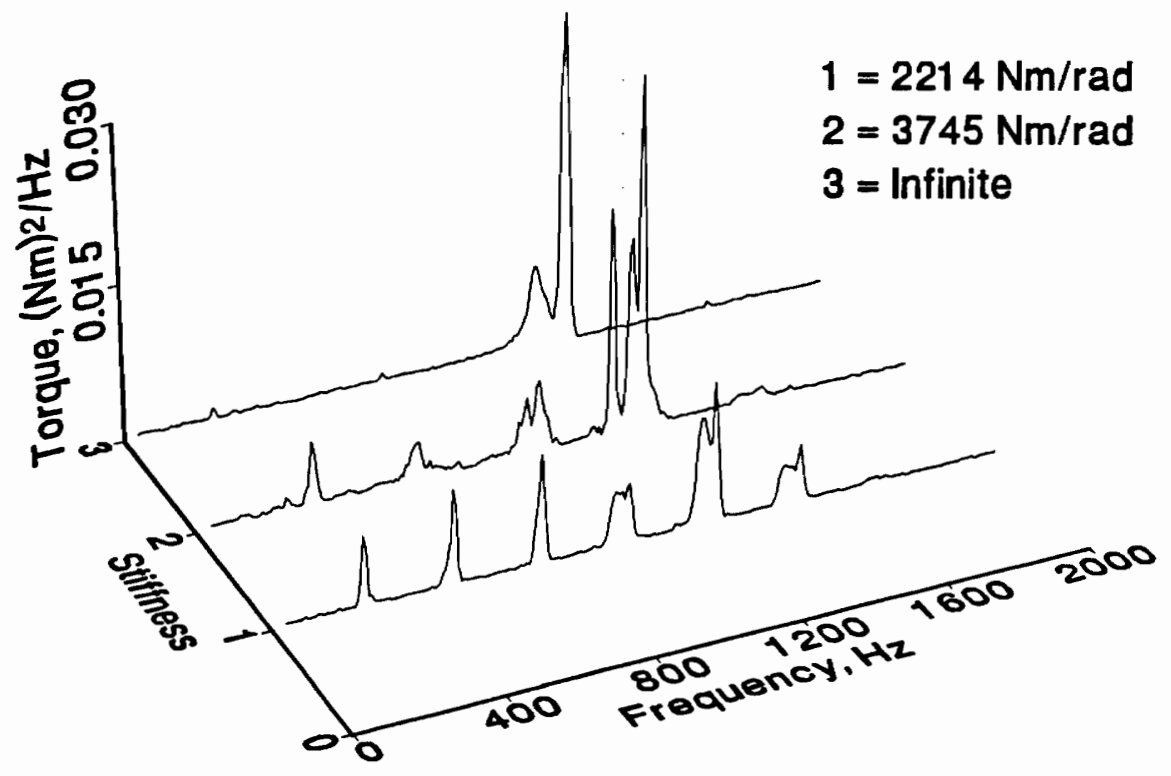

(a)

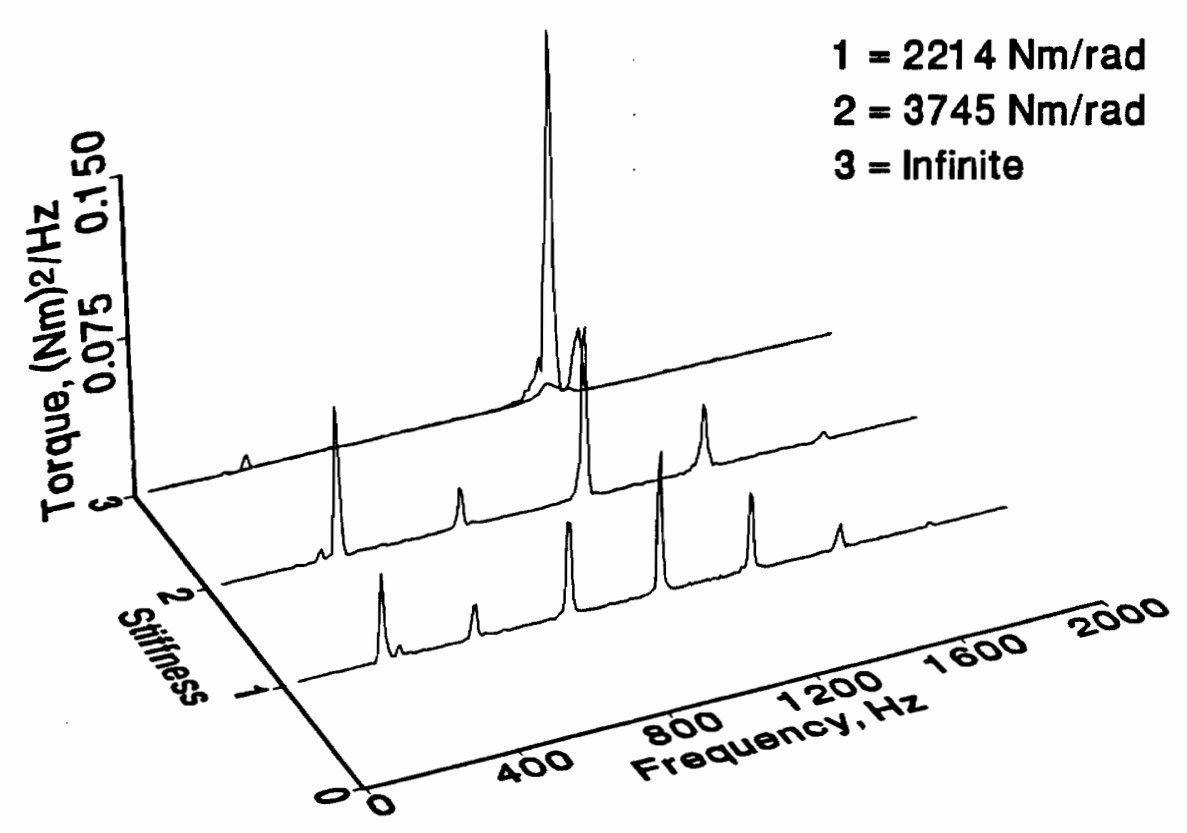

(b)

Figure 5.18 - Frequency content of the friction torque with increasing beam stiffness (a) $\delta=0.178 \mathrm{~mm}, \omega=2.5 \mathrm{rad} / \mathrm{s}(b) \delta=0.279 \mathrm{~mm}, \omega=7.5 \mathrm{rad} / \mathrm{s}$. 


\subsubsection{Friction Results-Natural Rubber}

\section{Effect of transfer film}

The vibration induced during sliding changed through the progression of experiments. The natural rubber bushing experienced a break-in period before consistent behavior was observed. As stated in Section 5.1.3 a variation precipitated from the formation of a transfer film and wear debris at the elastomer/shaft interface. The results of the vibration study will address the vibration characteristics for this break-in period, however, the primary emphasis will consider behavior after the transferred rubber was removed from the shaft.

Initial experiments reveal stick-slip behavior in the 5-10 rad/s velocity range (with $2,214 \mathrm{Nm} / \mathrm{rad}$ support stiffness and $0.127 \mathrm{~mm}$ interference fit). The autospectra at $\omega=$ 7 and $10 \mathrm{rad} / \mathrm{s}$ indicate slip occurs at $250 \mathrm{~Hz}$ corresponding to the system's first torsional vibration mode. The autospectra of the beam acceleration consistently contains peak frequency components at 250 and $900 \mathrm{~Hz}$. The superimposed frequency during the stick phase of the friction torque signal does not correspond to the $900 \mathrm{~Hz}$ frequency, however.

The formation of the transfer film increased the dc torque component three to four times. More important though, the transfer film altered the vibration behavior of the natural rubber bushing. At $\omega=5,7$, and $10 \mathrm{rad} / \mathrm{s}$ random vibration in the $250-900 \mathrm{~Hz}$ range was barely discernable in the noise threshold of the friction torque autospectral density. Despite the slightness of the friction induced vibration, the beams experienced 
an acceleration of approximately $50 \mathrm{~m} / \mathrm{s}^{2} \mathrm{rms}$. Again peak acceleration frequencies at 250 and $900 \mathrm{~Hz}$ were observed with an order of magnitude drop in amplitude.

Removing the transfer film from the shaft produced similar results to the initial experiments with this natural rubber specimen. Figure 5.19(a) shows a stick-slip oscillation at $250 \mathrm{~Hz}$ run at the same operating conditions as in Figure 5.7. Figure 5.19(b) is the autospectral density. The magnitude of the superimposed frequency is much less as is the magnitude of the $900 \mathrm{~Hz}$ component of the beam acceleration. Likewise, similar behavioral trends occurred before and after the transfer film at $5 \mathrm{rad} / \mathrm{s}$ (see Figure 5.8). At $1 \mathrm{rad} / \mathrm{s}$, steady state sliding was observed.

\section{Effect of system dynamics}

Increasing the system support stiffness to $3,745 \mathrm{Nm} / \mathrm{rad}$ had two predominant effects. The slip frequency increased from 250 to $310 \mathrm{~Hz}$. Figure 5.20(a) and (b) shows the resulting frequency shift in the autospectra. Likewise the peak frequency components of the beam acceleration shifted from 250 and $900 \mathrm{~Hz}$ to 310 and $1375 \mathrm{~Hz}$. The other influence observed was that stick-slip was evident at $1 \mathrm{rad} / \mathrm{s}$. Prior to and during experiments with the transfer film, steady sliding was observed at this angular velocity.

\subsubsection{Acoustic Results}

\section{Fluorocarbon}

Experiments in the 1-10 rad/s velocity range produced three types of audible noise output. These different types of noise were subjectively labeled creak, squawk, and 


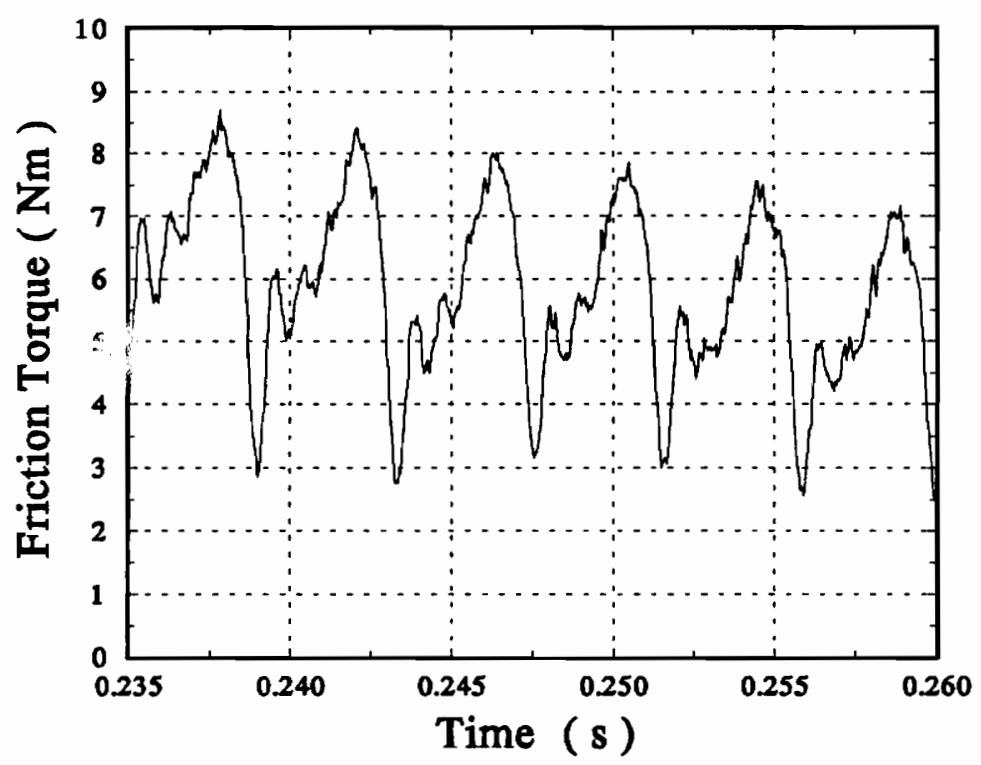

(a)

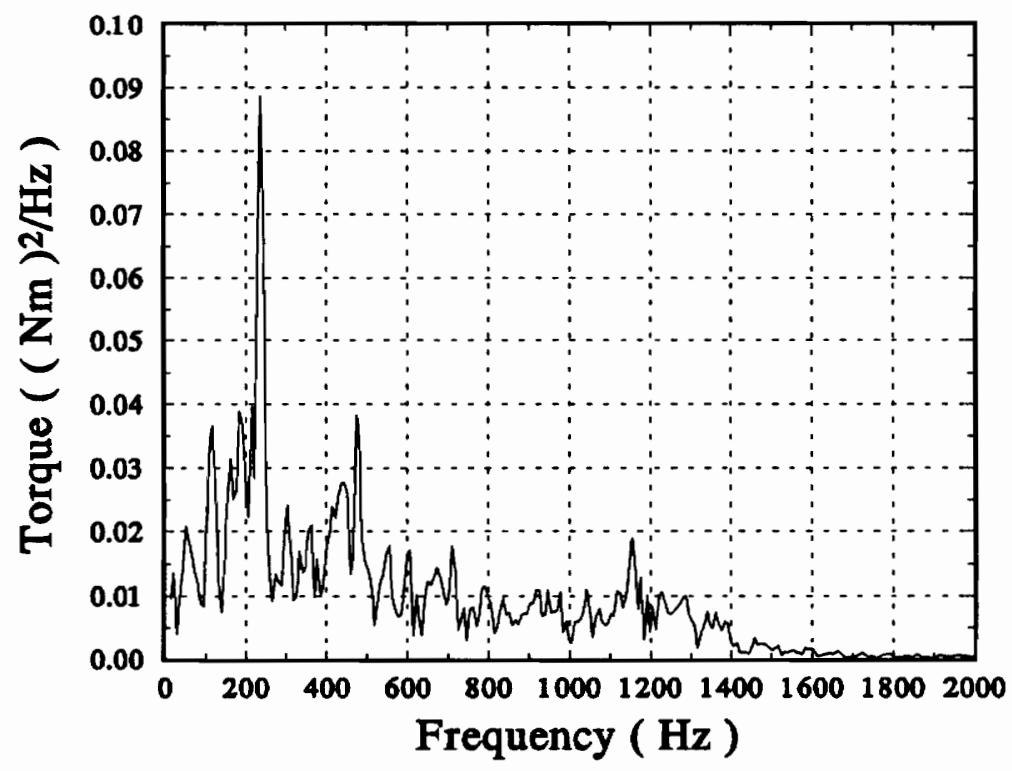

(b)

Figure $5.19-$ Natural rubber friction behavior $k_{B}=2,214 \mathrm{Nm} / \mathrm{rad}$, $\delta=0.127 \mathrm{~mm}$ ) (a) torque time history and (b) autospectral density. 


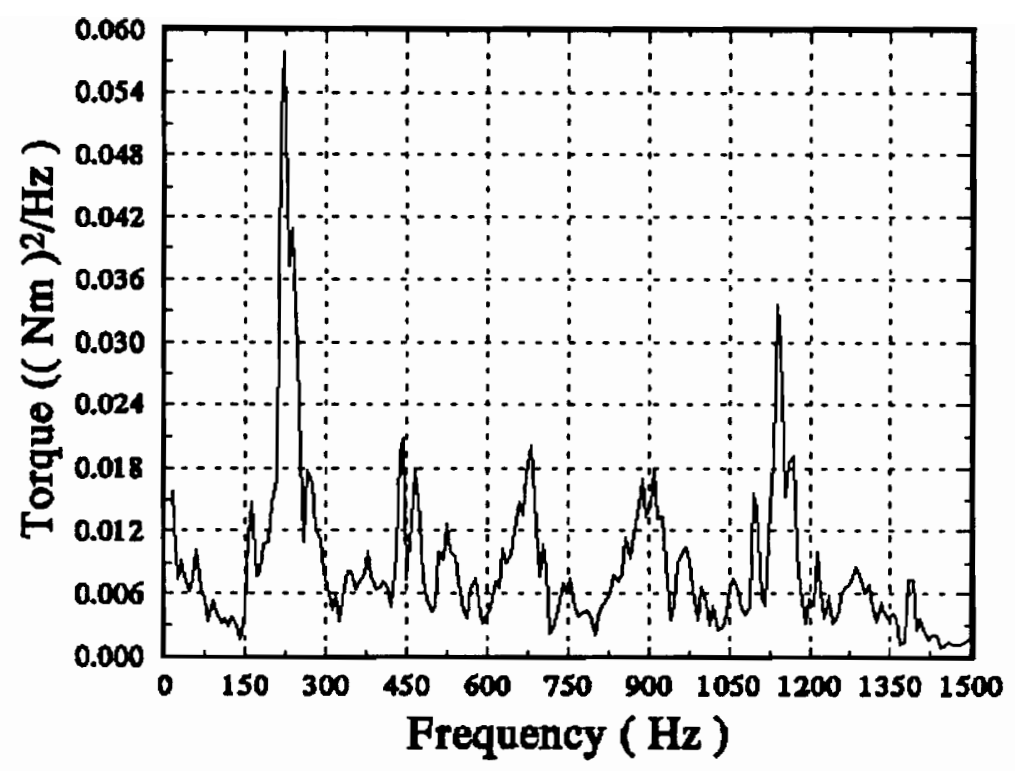

(a)

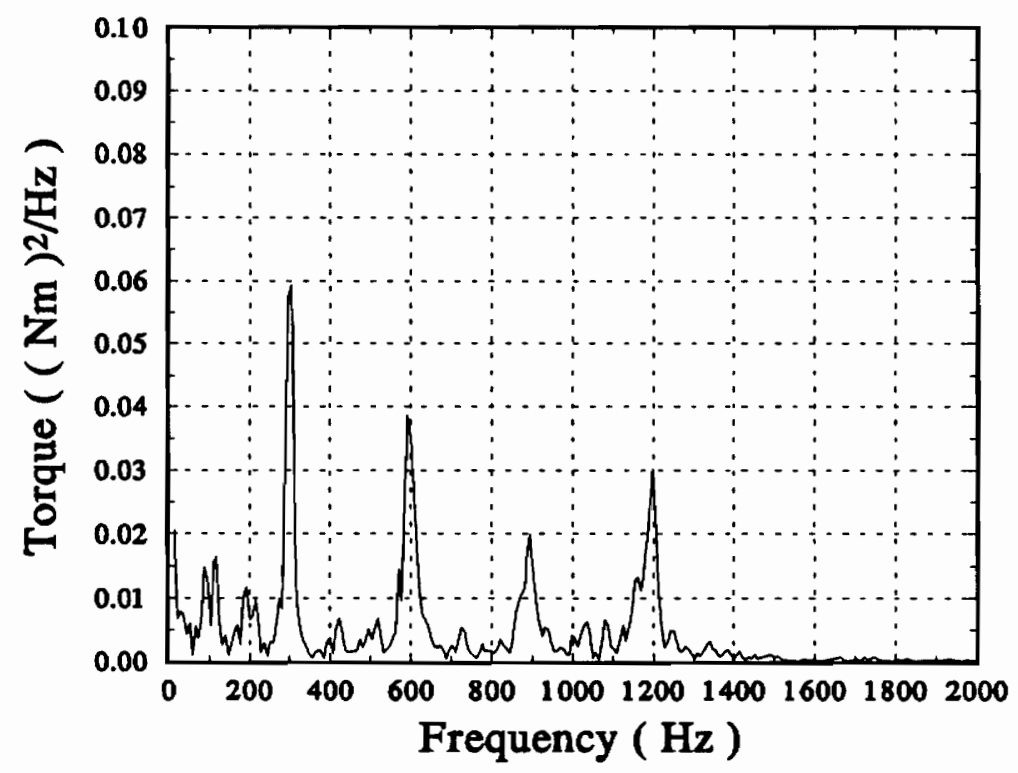

(b)

Figure 5.20-Natural rubber friction behavior $(\omega=10 \mathrm{rad} / \mathrm{s}, \delta=0.127$ mm) (a) $k_{B}=2,214 \mathrm{Nm} / \mathrm{rad}$ and (b) $k_{B}=3,745 \mathrm{Nm} / \mathrm{rad}$. 
squeal. Velocity, system stiffness, and interference fit were varied to see if the audible noise content of sound pressure was influenced in conjunction with friction induced vibration. Analyses of time histories in the frequency domain were made to show the differences between the system torque and beam acceleration for each type of noise produced.

Creaking noise was generated only at the largest interference fit $(\delta=0.381 \mathrm{~mm})$ for the fluorocarbon material. Figure 5.21(a)-(f) illustrates the torque, beam acceleration, sound pressure and their associated autospectra during this type of noise generation for an experiment run at $\omega=1 \mathrm{rad} / \mathrm{s}, k_{B}=2,214 \mathrm{Nm} / \mathrm{rad}$, and $\delta=0.381$. Creak noise was characterized by sound pressure frequencies ranging from the stick-slip frequency through $15 \mathrm{kHz}$. The instant slip occurs is indicated in Figure 5.21 (b) and (c) by the largest amplitude in the acceleration and sound pressure signals. After the slip event the beams drive the plate and acoustic energy is radiated.

The noise output appeared to get louder as the shaft velocity was increased. The sound pressure amplitude did not increase with increasing shaft velocity, however the number of excitations per unit time increased, which increased the root mean square pressure radiated from the vibrating system. Increasing the system stiffness from 2,214 to $3,745 \mathrm{Nm} / \mathrm{rad}$ created higher stick-slip frequencies and thus higher fundamental acoustic frequencies. Peak sound pressure amplitudes decreased by approximately 25 percent in the time domain by increasing the system stiffness. The amplitudes in the frequency domain from $10-15 \mathrm{kHz}$ decreased slightly. When the clamp was fixed to the baseplate, the frequency content consisted strictly of the stick-slip frequency and its 

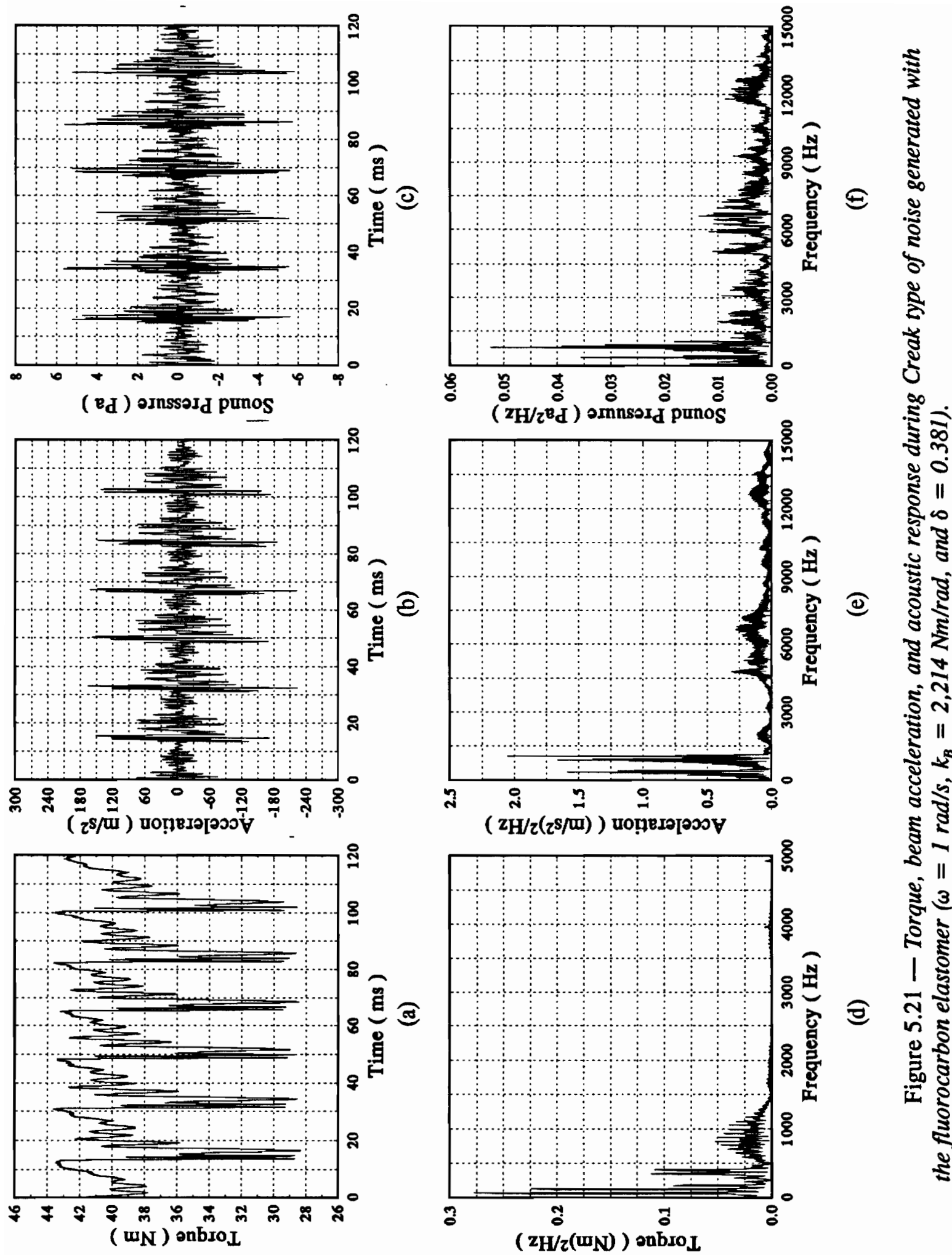
harmonics.

Squawk type noise was generated when the interference fit was 0.178 and 0.279 mm. Squawk occurred in the quasi-harmonic friction regime when the torque consisted of more than one discrete frequency. This type of acoustic output was observed at $\delta=$ $0.278 \mathrm{~mm}$ and shaft velocities ranging from 5-7.5 rad/s. Figures 5.22(a)-(f) illustrates the torque and beam acceleration behavior when this type of noise is generated. Figures 5.22(d)-(f) show the replication of torque frequencies in the acceleration and acoustic autospectra. The frequencies in the autospectra increase when the beam stiffness is changed from 2,214 to $3,745 \mathrm{Nm} / \mathrm{rad}$ as shown in Figure 5.23(a)-(f). At $\delta=0.178 \mathrm{~mm}$ and $k_{B}=2,214$, this type of noise occurred in the 1-4.6 rad/s velocity range. The peak frequencies of the friction and acceleration consisted of the stick-slip frequency and harmonics through $1250 \mathrm{~Hz}$. This range extended to $1750 \mathrm{~Hz}$ with beam stiffness at $3745 \mathrm{Nm} / \mathrm{rad}$.

Squeal noise was generated at lower pressures and high shaft velocities. Squeal was observed when the friction torque appeared to be harmonic or triangular in nature as in Figure 5.1(c) and (d). This behavior is confirmed by single or predominant peaks in the acceleration and sound pressure autospectra. Figure 5.24(a)-(d) shows single frequencies at $6570 \mathrm{~Hz}$ were present at $\omega=10 \mathrm{rad} / \mathrm{s}, k_{B}=3745 \mathrm{Nm} / \mathrm{rad}$, and $\delta=$ $0.279 \mathrm{~mm}$. Similar behavior happened when the interference fit was $0.178 \mathrm{~mm}$.

PDMS

Friction induced oscillations did not arise when the shaft was rotated inside the PDMS bushing. The absence of vibration in the torque and beam acceleration signals 

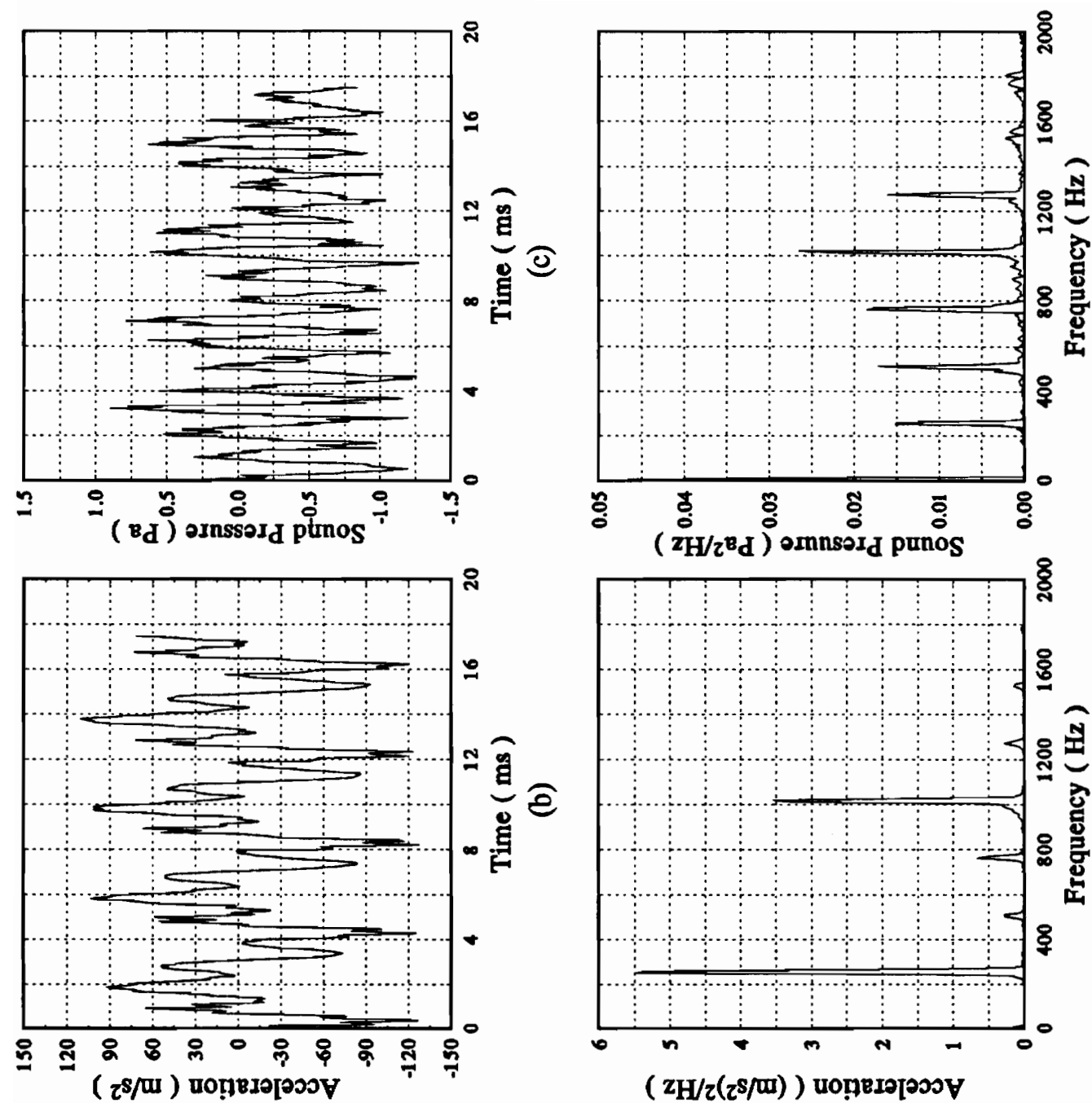

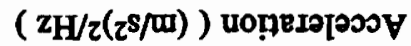
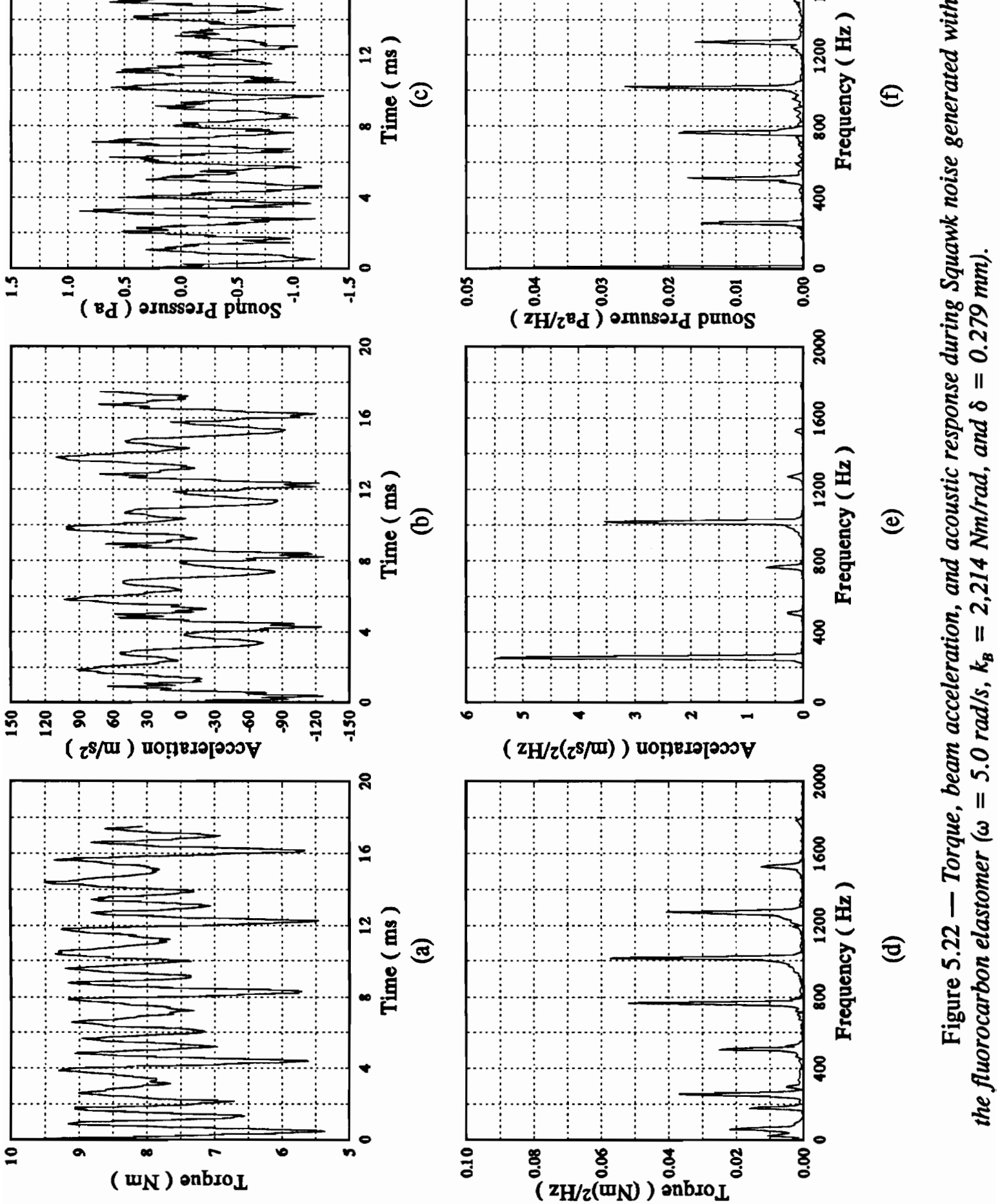

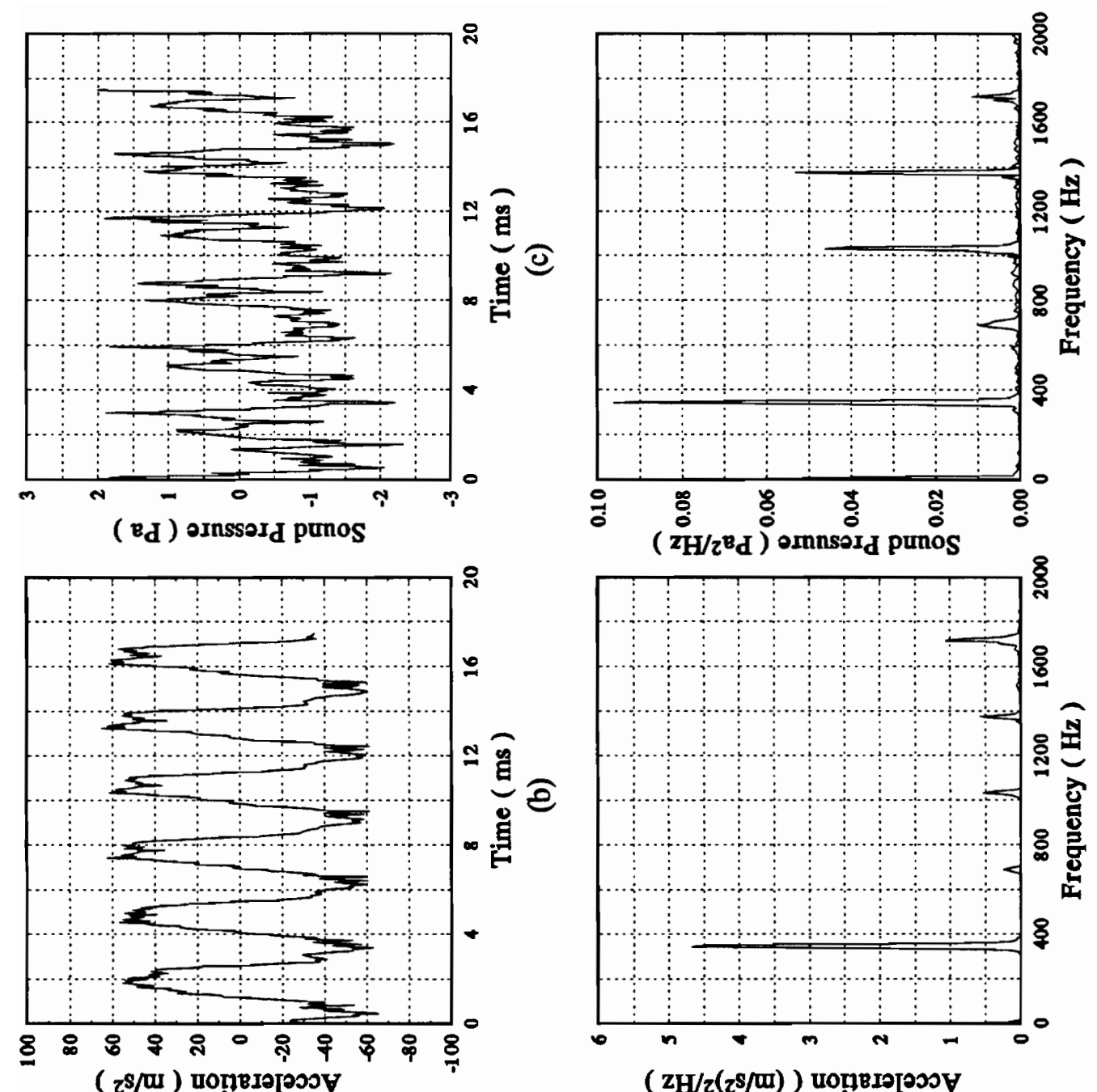

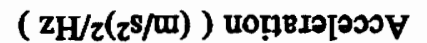
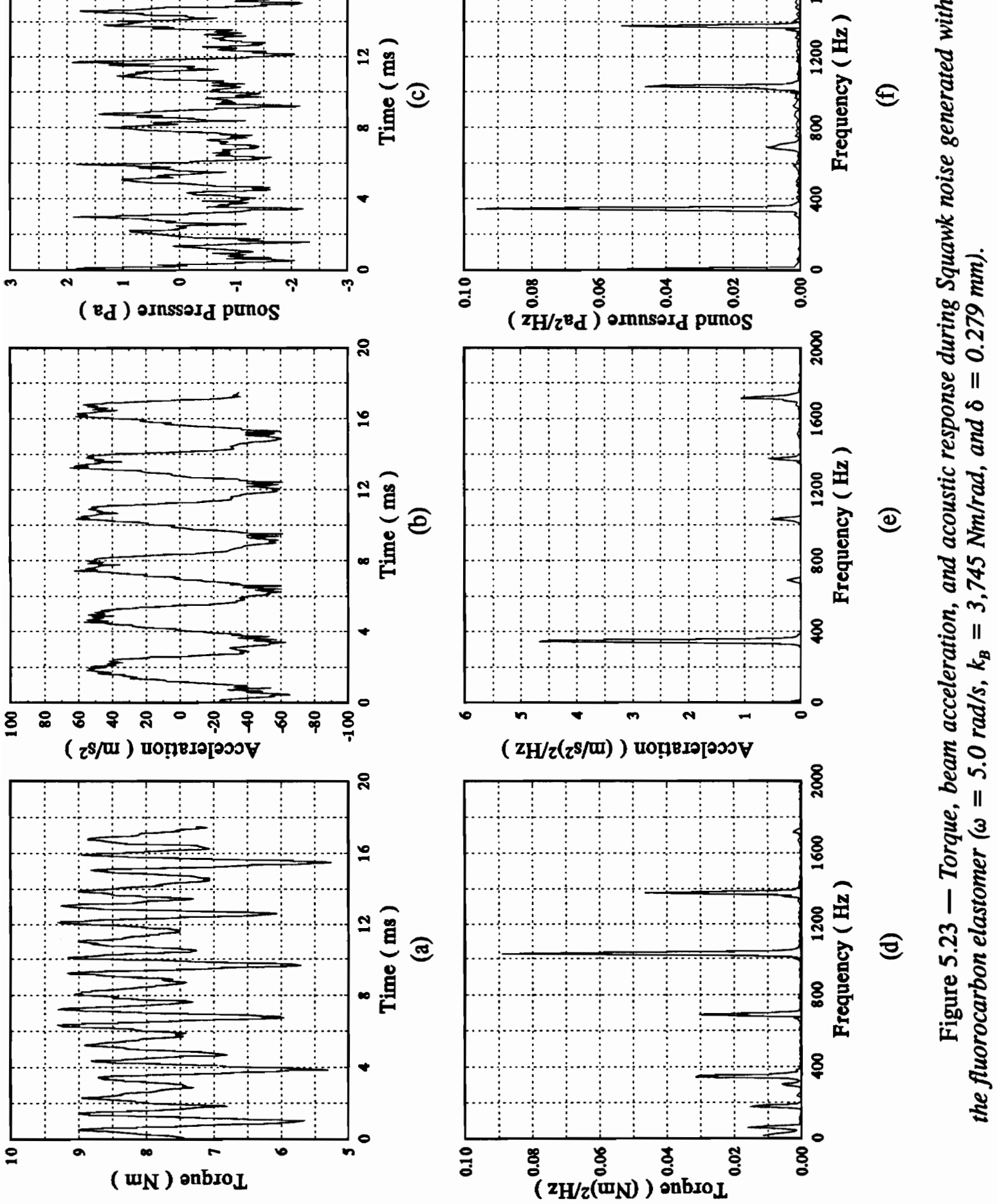

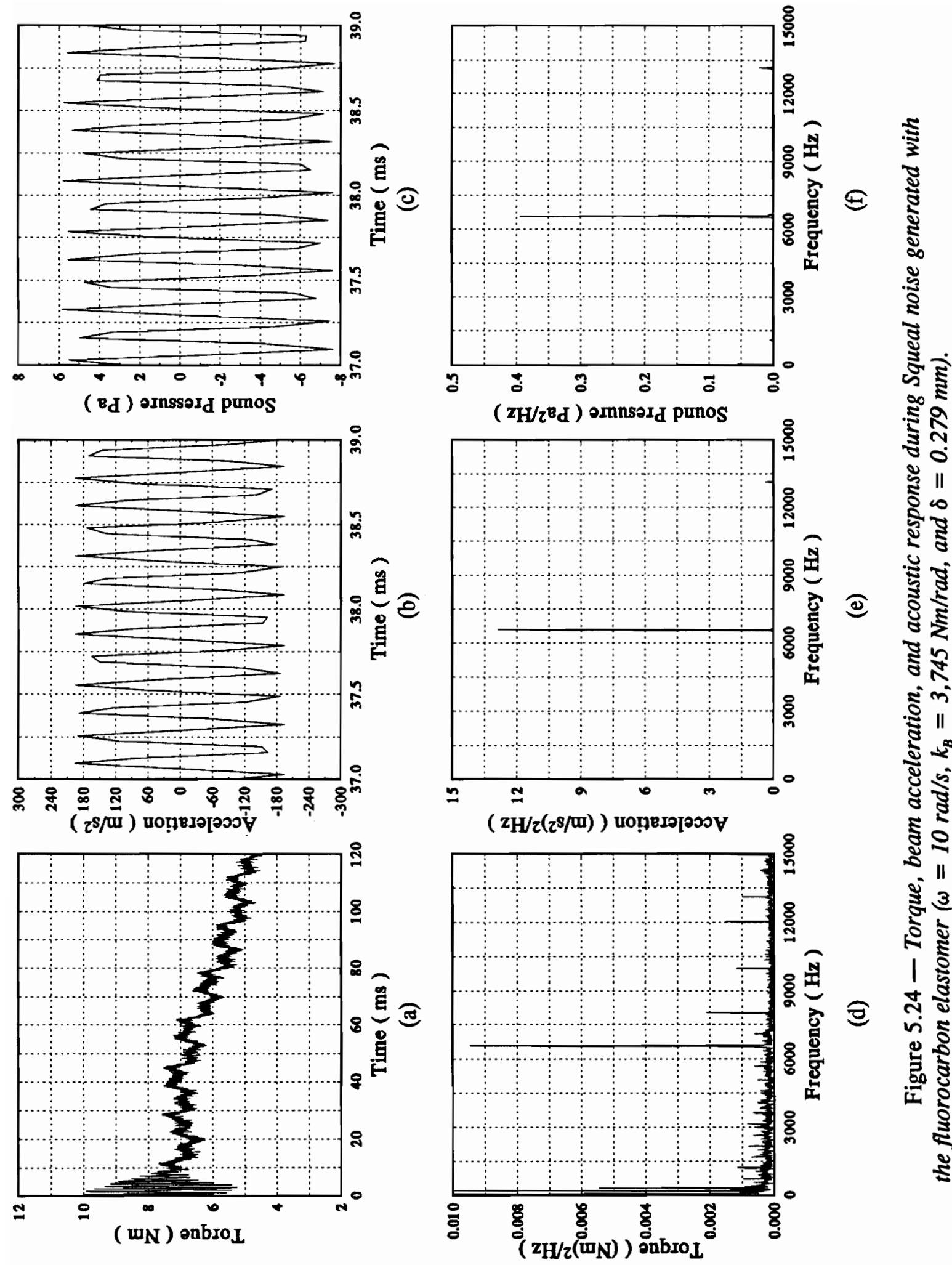
made noise generation impossible for this material at the experimental operating conditions.

\section{Natural rubber}

The formation of a transfer film during experiments with this material caused many changes in torque and acoustic behavior. The noise that was produced by the frictional sliding of this material changed in parallel with the vibration behavior discussed in Section 5.2.2. Before the transfer film developed, the sound pressure was composed of peaks coinciding with the stick-slip frequency. This frequency corresponded to the first torsional mode of system vibration. Other frequencies present in the sound pressure autospectrum did not correlate with those in the torque or acceleration autospectra. Experiments conducted with the transfer film established showed random friction vibration in the $250-1500 \mathrm{~Hz}$ range with very small amplitude. The acceleration showed small amplitude vibration at the natural modes of the system $(250,900,4500$, and 6000 $\mathrm{Hz}$ ). The sound pressure autospectrum did not contain the peaks that were prevalent in the beam signal but instead consisted of random noise from 100 to $6000 \mathrm{~Hz}$.

After the transfer film was removed from the shaft, the acoustic output change significantly. Experiments with $k_{B}=2,214 \mathrm{Nm} / \mathrm{rad}$ and velocities ranging from 1-19.75 $\mathrm{rad} / \mathrm{s}$ indicate predominant frequencies in the torque also are seen in the acceleration and sound pressure autospectra. The sound pressure autospectrum also contained random noise through $2 \mathrm{kHz}$. The beam stiffness was then increased to $3,745 \mathrm{Nm} / \mathrm{rad}$ increased the stick-slip frequency slightly. The sound pressure for this test condition coincided with the first torsional mode of system vibration which was excited by the random 
friction induced vibration. 


\subsection{Torsional Spring Constants and Damping Ratios}

Results from the torsional spring constant measurements indicate linear spring rates exist until slip occurs. Measurements were taken at $0.279 \mathrm{~mm}$ and $0.381 \mathrm{~mm}$ interference fits and three beam stiffness conditions. The effective spring rates and elastomer spring rate for each pressure condition are plotted in Figures 5.25 and 5.26.

The effective torsional spring rate, $k_{E F F}$, for each beam length was determined via linear regression of the static torque versus radial deflection curves. The torsional compliance $k_{E}$ was found in a similar manner. The slopes from each regression are listed in Table 5.3.

Table 5.3. Torsional spring rates of system and fluorocarbon elastomer.

\begin{tabular}{|c|c|c|c||}
\hline $\begin{array}{c}\delta \\
{[\mathrm{mm}]}\end{array}$ & $\begin{array}{c}k_{\text {EFF }}[\mathrm{Nm} / \mathrm{rad}] \\
@ k_{B}=2,214\end{array}$ & $\begin{array}{c}k_{\text {EFF }}[\mathrm{Nm} / \mathrm{rad}] \\
@ k_{B}=3,745\end{array}$ & $\begin{array}{c}k_{E}[\mathrm{Nm} / \mathrm{rad}] \\
\text { Fixed }\end{array}$ \\
\hline \hline 0.279 & 464 & 576 & 665 \\
\hline 0.381 & 587 & 764 & 1011 \\
\hline
\end{tabular}

Theoretical values of the effective spring rate for each system configuration and interference fit were determined using the relation

$$
k_{E F F_{T A B O}}=\left[\frac{1}{k_{B_{T H B O}}}+\frac{1}{k_{E_{E X P}}}\right]^{-1}
$$




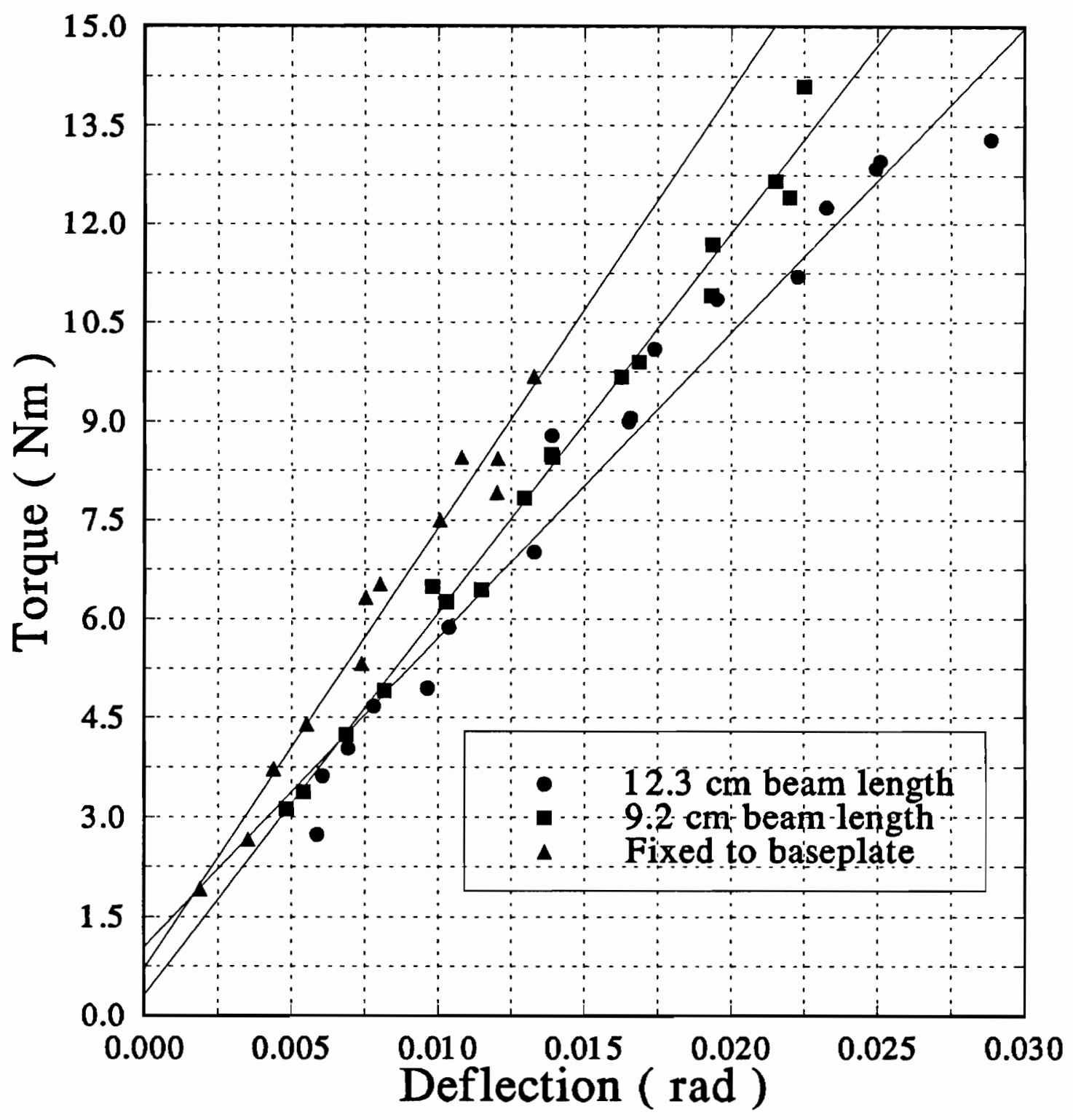

Figure 5.25 - Static deflection versus torque data with linear regression curve fit with $0.279 \mathrm{~mm}$ interference fit. 


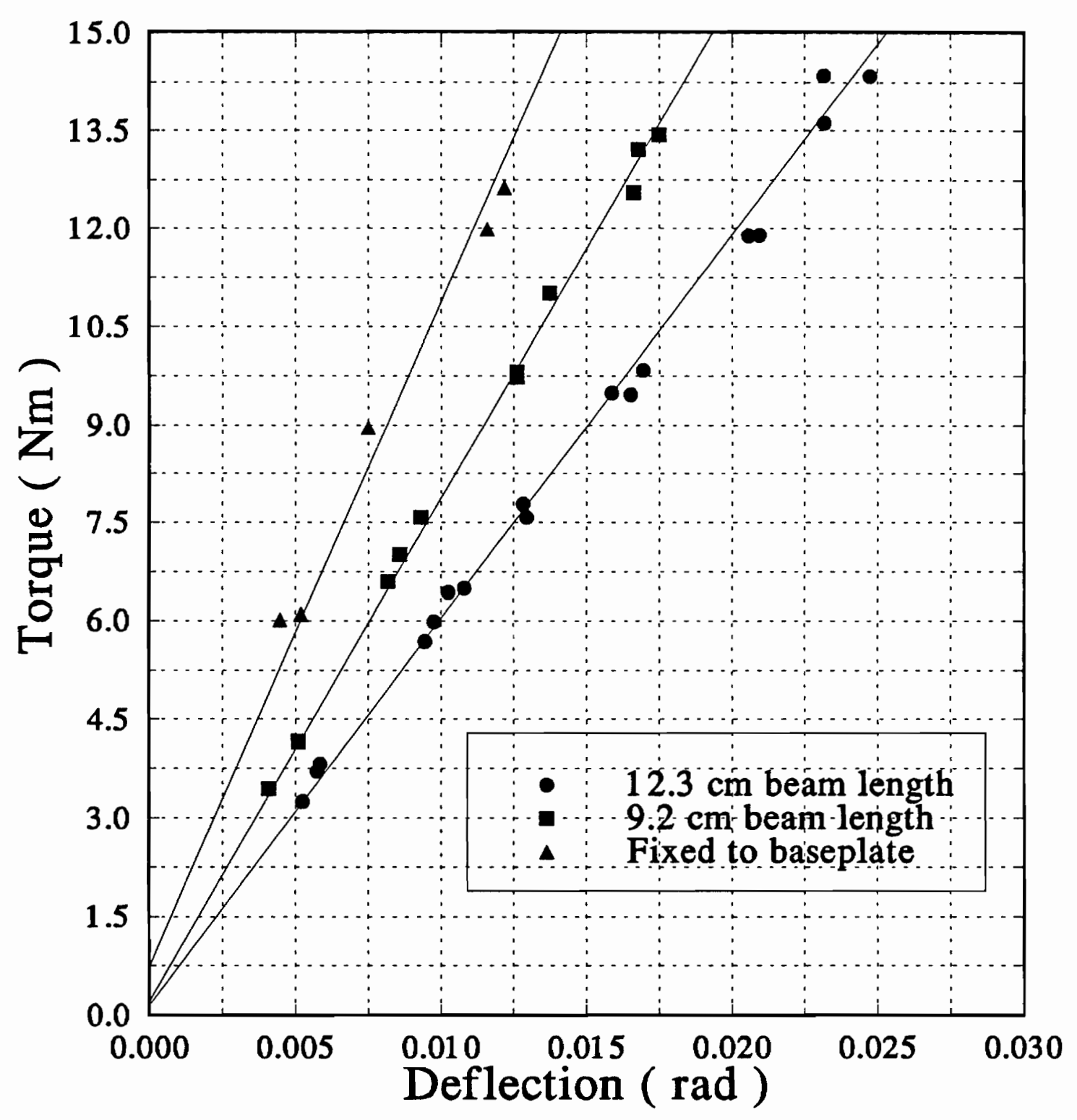

Figure 5.26 - Static deflection versus torque data with linear regression curve fit with $0.381 \mathrm{~mm}$ interference fit. 
where $k_{\text {Впво }}$ is the theoretical torsion beam stiffness, $k_{B}$, derived in Section 4.3, and $k_{E \text { exr }}$ is the experimental torsional spring rate of the elastomer. Results indicate a range of accuracy for the effective spring rates within two to fifteen percent.

Table 5.4. Torsional spring rates of system and fluorocarbon elastomer.

\begin{tabular}{|c|c|c||}
\hline \multirow{2}{*}{$\begin{array}{c}\delta \\
\mathrm{mm}]\end{array}$} & \multicolumn{2}{|c|}{ Experimental Error, $\epsilon$} \\
\cline { 2 - 3 } & \hline$k_{B}=2,214[\mathrm{Nm} / \mathrm{rad}]$ & $k_{B}=3,745[\mathrm{Nm} / \mathrm{rad}]$ \\
\hline \hline 0.279 & $9.3 \%$ & $2.0 \%$ \\
\hline 0.381 & $15.4 \%$ & $4.0 \%$ \\
\hline
\end{tabular}

The damping coefficient, $c_{E}$, was determined experimentally for the aforementioned system stiffnesses. The system's impulse response was used to calculate $c_{E}$. Viscous damping causes the vibration of an underdamped system to decay exponentially as a function of time. The system described by the second order, homogeneous equation

$$
J \ddot{\theta}+c \dot{\theta}+k \theta=0
$$

has the general solution [26]

$$
\theta-C_{1} e^{s_{1} t}+C_{2} e^{s_{2} t}
$$

where $C_{1}$ and $C_{2}$ are constants which are evaluated with initial conditions. Substituting the eigenvalues $s_{1}$ and $s_{2}$ into equation 3 gives

$$
\theta=e^{(-c / 2 \pi) t}\left(C_{1} e^{\sqrt{(c / 2 J)^{2}-(k /) t} t}+C_{2} e^{\sqrt{(c / 2 J)^{2}-(k /)} t}\right)
$$


The above equation may also be written in the form

$$
\theta-\theta e^{(-c / 2 J) t} \sin \left(\sqrt{1-(c / 2 J)^{2}} \omega_{n} t+\phi\right)
$$

where the curves

$$
\theta- \pm \theta e^{(-c / 2) t}
$$

envelope the decayed response.

The peak amplitudes from each cycle of the vibration response were plotted as a function of time. An exponential curve fit was generated for each response. The damping coefficients, $c_{E}$, were determined for each support stiffness and interference fit and are shown in Table 5.5. The damping coefficient at $k_{B}=2,214$ and $\delta=0.381 \mathrm{~mm}$ is larger than the other coefficients. This was due to a beat phenomenon occurring at this system configuration.

Table 5.5. Torsional damping coefficients of system and fluorocarbon elastomer.

\begin{tabular}{|c|c|c||}
\hline \multirow{2}{*}{$\begin{array}{c}\delta \\
{[\mathrm{mm}]}\end{array}$} & \multicolumn{2}{|c|}{ Experimental Damping Coefficient, $c_{E}$} \\
\cline { 2 - 3 } & $k_{B}=2,214[\mathrm{Nm} / \mathrm{rad}]$ & $k_{B}=3,745[\mathrm{Nm} / \mathrm{rad}]$ \\
\hline \hline 0.279 & $0.105\left[\mathrm{kgm}^{2} / \mathrm{rad} / \mathrm{s}\right]$ & $0.085\left[\mathrm{kgm}^{2} / \mathrm{rad} / \mathrm{s}\right]$ \\
\hline 0.381 & $0.236\left[\mathrm{kgm}^{2} / \mathrm{rad} / \mathrm{s}\right]$ & $0.080\left[\mathrm{kgm}^{2} / \mathrm{rad} / \mathrm{s}\right]$ \\
\hline
\end{tabular}




\subsection{Discussion}

\subsection{Friction Behavior}

The sliding velocity was the most significant factor affecting friction behavior. Three distinct regimes of friction were observed as the velocity increased. Stick-slip generally occurred at low velocities because of slow loading rates and small relative velocity changes between the shaft and elastomer. Quasi-harmonic friction was observed when the energy in the system was insufficient to restore the relative velocity to zero. The quasi-harmonic torque behavior was sustained by energy transfer from the beam and elastomer to the interface. Steady state friction occurred at high rotation velocities. Steady sliding either resulted because the damping was too high to sustain oscillation or not enough energy could be stored in the system to initiate vibration.

Altering the interference fit, $\delta$, invoked many changes in the frictional behavior. Increasing $\delta$ increased the mean friction torque levels as expected. It also caused a decrease in stick-slip frequency because more time was required to reach the maximum allowable shear load of the interface. The effects of interference fit on amplitude and frequency content shown in Figure 5.15 are similar for all stiffness and velocity conditions where friction vibration was observed.

The slip amplitudes were larger at $\delta$ of $0.278^{\prime \prime}$ and $0.381^{\prime \prime}$ because more energy was stored in the beams prior to slip. The larger interference fits also caused the transition from stick-slip to harmonic oscillation to occur at higher sliding speeds. The 
reason for this can be explained by analyzing the system interaction during the slip phase. When the elastomer slips, the structure will accelerate to its new equilibrium position at the system's damped natural frequency. The beams and the elastomer would contribute to the damping of the system. During this time, the relative velocity of the elastomer and shaft will increase from zero and then return to zero, thus ending the slip phase of the cycle. Therefore, if the shaft velocity is sufficiently high and the potential energy stored in the system is not great enough to restore the relative velocity to zero, the stick phase cannot resume.

Several changes in frictional behavior were observed as the support stiffness was changed. The amplitude of oscillations did not change while maintaining constant velocity and pressure, but the frequency content changed considerably. Increasing the torsional stiffness increased the stick-slip frequency and the frequency of the oscillations during the stick phase. This oscillation arises from the feedback of beam vibration that is transmitted through the clamp and bushing to the elastomer/shaft interface. The stiffer beam setting increased the frequency of the quasi-harmonic vibration but did not influence the frequency as much as changes in velocity. Preventing clamp oscillation about the shaft caused the sawtooth oscillation.

To ensure frictional heating during the experiments did not affect the storage and loss moduli of the elastomer a one-dimensional, conductive heat transfer model was developed. The elastomer and steel shaft were modeled as a semi-infinite plane wall. One dimensional heat conduction assured the most conservative estimate. The heat rate was derived from the product of the torque, $T$, and angular velocity, $\omega$. This was 
divided by the contact area, $A_{\text {conact }}$, to establish the overall heat flux at the interface. Thermal properties dictate $92 \%$ of the energy will be conducted by the steel shaft and $8 \%$ by the elastomer. The most severe experimental conditions with regard to temperature is for the fluorocarbon with $T=30 \mathrm{Nm}$, test duration $t=.23 \mathrm{sec}, A_{\text {contect }}$ was assumed to be $0.2 A_{\text {apparent }}$, and $\omega=10 \mathrm{rad} / \mathrm{s}$. The surface temperature rise was 26 ${ }^{\circ} \mathrm{C}$. The temperature $0.0004 \mathrm{~mm}$ into the bulk elastomer was $22{ }^{\circ} \mathrm{C}$ which was ambient temperature. These calculations and the observation that the friction characteristics did not change from start to finish in each experiment indicate temperature did not influence the mechanical properties of the elastomer. The conductive heat model derivation and plots of the temperature calculation are included in Appendix G.

The difference in the friction behavior of the fluorocarbon and that of the PDMS and the natural rubber can be explained by the relative magnitudes of the adhesion and deformation components of friction for each polymer. The fluorocarbon has greater hardness, strength, and adhesion as determined from O-ring seal tests [27]. One could propose that the adhesion term will dominate the magnitude of the friction torque. This bushing was scrutinized for wear. Cohesive failure of the elastomer and shaft surfaces was indicated by pitting of the fluorocarbon and the removal of flecks of the epoxy paint from the shaft. The roughness was not significantly changed during the test from its initial value of 5-10 $\mu \mathrm{m} \mathrm{R}$. The independence of torque with velocity is evidence that either the deformation term is negligible in comparison with the adhesion, or the velocity range of the experiments is too small for the viscoelastic properties of the fluorocarbon to affect its friction behavior. The fluorocarbon exhibited the most well defined 
frequency content of torque and system vibration. Strong adhesion caused contact regions to form and reach maximum shear simultaneously, resulting in periodic slip of the entire bushing/shaft interface.

Natural rubber behavior resembled that of the fluoro-elastomer. However, the torque frequency content indicated the presence of random noise other than at the slip frequencies. The contact regions probably did not form synchronously for this material as might have occurred for the fluorocarbon. The natural rubber friction torque was similar to that of the fluorocarbon elastomer; it was independent of angular velocity over the range of experiments. The latter comment and the significant wear of the bushing (none on the shaft, though) imply the adhesion mechanism predominated in this velocity range.

PDMS has lower adhesion, shear strength, and hardness. Increasing the interference fit from $0.254 \mathrm{~mm}$ to $0.381 \mathrm{~mm}$ did not increase the magnitude of the friction. This suggests a maximum real contact area was attained at the lower pressure and friction behavior is dominated by the deformation due to the sliding of macroasperities through the material. The magnitude of the friction was dependent on velocity suggesting that deformation or loss modulus dictated the friction/velocity relationship. Physical evidence supporting the dominance of deformation over adhesion was an absence of transfer film on the shaft and no wear scars on the bushing surface.

Despite the differences between material behavior, some generalizations can be made. Increasing the velocity and the system stiffness increases the stick-slip frequency which did not exceed the frequency of the first torsional mode of the system, however. 
The materials exhibiting frictional instability were able to impart sufficient potential energy to the supporting structure to influence the frequency content of the friction torque when the energy was converted to kinetic energy during a slip phase. Friction instability was not observed during PDMS experiments because PDMS had a positive friction/velocity trend and did not exhibit a difference in static and kinetic friction. For this reason changing system stiffness did not affect the frictional behavior. 


\subsection{Conditions Required for Noise Generation}

The sound generated during these experiments varied with interference fit, system stiffness, and bushing material. This happened because the response and the acoustic paths were different for each type of self-excited oscillation. At certain operating conditions there was a one-to-one matching of frequencies between the torque and sound pressure and at other times no correlation was apparent. When and why the quality of the noise differed will be discussed in this section.

Sound output from the fluorocarbon experiments differed to a great extent. This was due to the variety of oscillation types and the amplitude of oscillation. Generally squeal noise was marked by single coinciding frequency peaks in the torque, beam acceleration, and sound pressure autospectra. There were occasions when other lower amplitude frequencies were present in the acceleration signal, but only one predominated and this made only a squeal sound apparent to the listener. Since the friction vibration amplitude was relatively small for the harmonic oscillation seen in the torque and acceleration, the frequency response of the beam system must have been such that the other normal modes of the beam were not excited. From Figure 5.24 it appears the primary acoustic path is sound radiated from the interface and the beams.

Figures 5.22 and 5.23 indicate the common frequencies of the torque, acceleration, and sound pressure response of squawk. This behavior is similar to that which occurred with squeal except several harmonics are present in each autospectrum. Conversely, when creak sound was heard the beam acceleration autospectrum contained 
peaks at what appear to be natural modes of the apparatus and the sound pressure consisted of broad band noise from the stick-slip frequency through $15 \mathrm{kHz}$. The torque did not contain frequencies past $2 \mathrm{kHz}$ so the noise had to come from another source. It was discovered to be generated from the plate. Thus sound pressure was not only generated by the vibration of the beams, but also from the plate which was driven by vibration transmitted through the beam anchors to the plate.

The stick-slip torque behavior observed at $\delta=0.278$ and $0.381 \mathrm{~mm}$ did not have different wave forms or contain higher frequencies. However, when creaking noise occurred at the higher interfacial fit, the amplitude of vibration was greater. In order to resolve where the unaccounted frequencies not found in the beam acceleration, the acoustic response of the plate was experimentally determined. A modal hammer was used to impulsively excite the apparatus. The microphone over the plate sensed the acoustic response of the apparatus. The frequency response of the system was calculated for each beam configuration. The responses are plotted in Appendix $\mathrm{H}$.

The response of the system show frequencies over the entire $15 \mathrm{kHz}$ range. From these figures it is apparent that when creaking sound is generated, the larger slip amplitude adds enough energy in the beam system to drive the plate. Thus it is not necessarily the type of friction behavior observed that dictates the type of audible noise generated but the magnitude of vibration it imparts to the system.

Chatter noise produced with the natural rubber specimen were similar to the squawk noise produced by the fluorocarbon. This phenomenon occurs during stick-slip with no transfer film present. The peak frequencies in sound pressure consistently 
coincided with the stick-slip frequency. The only correlation between the acceleration and sound pressure is when the stick slip frequency is at the first natural vibration mode of the beams. During chatter of the natural rubber bushing, random noise is also present in the autospectrum of sound pressure up to approximately $1,750 \mathrm{~Hz}$.

The sound generated when the natural rubber transfer film was present resembled the fluorocarbon creaking sound except it was not as loud. The torque was comprised of low amplitude, random noise from $25-1,500 \mathrm{Khz}$. The beam acceleration vibrated at the natural modes of $25,900,4,500$, and $6,000 \mathrm{~Hz}$. This combination of vibration caused random noise up to $6,000 \mathrm{~Hz}$ in the sound pressure signal. 


\subsection{Comparison of Simulated and Experimental Behavior}

The friction torque and system behavior was simulated for the operating conditions of the fluorocarbon elastomer experiments. This includes angular velocities ranging from $1-10 \mathrm{rad} / \mathrm{s}$, interference fits of 0.279 and $0.381 \mathrm{~mm}$, and system stiffnesses of 2,214 and $3,745 \mathrm{Nm} / \mathrm{rad}$. The simulations required a $0.2 \mathrm{~ms}$ time step for accurate solution of the differential equations. For each set of operating conditions, the simulation spanned $82 \mathrm{~ms}$. A few simulations of $820 \mathrm{~ms}$ were obtained for accurate spectral averaging.

\subsubsection{Friction/velocity Results of Simulation}

Stick-slip, quasi-harmonic, and harmonic oscillations were observed from simulation data. For the stiffness case, $k_{B}=2,214 \mathrm{Nm} / \mathrm{rad}$, stick-slip was observed through $\omega=7.5 \mathrm{rad} / \mathrm{s}$. Stick-slip occurred through $\omega=5 \mathrm{rad} / \mathrm{s}$ when $k_{B}=3,745$. The transition velocities are much higher in simulation than the experimental results indicate. This is due to the assumption in the simulation that the interface is entirely stuck during the stick phase. In reality, this is not true. In experiment the torque does not increase linearly with time as in the simulations. At the beginning of the stick phase, the entire contact region has zero relative velocity. As the strain on the adhered contact region increases, fractions of the contact region break free allowing relative motion between the elastomer and shaft. As the adhered contact region decreases in size the rate of torque increase must also decrease since there is less area to support the static load. The 
magnitude of the $V_{\text {cril }}$ aside, the trends of friction regimes with increasing stiffness agree with experimental results in Table 5.1.

Quasi-harmonic oscillations occurred when the rate of system torque increase equalled the rate of torque decrease during free vibration of the system. This behavior is stick-slip minus the superimposed frequency during the stick phase. It should be noted that the harmonic oscillations were not self-sustained oscillations but were the result of the step response of the system as the friction falls from $T_{c r i s}$ to $T_{k i n}$.

Figure 6.1 shows the system torque, displacement, and relative angular velocity curves for the conditions $\omega_{\text {shaft }}=1 \mathrm{rad} / \mathrm{s}, k_{B}=3,745$, and $\delta=0.279 \mathrm{~mm}$. The slip event is triggered at approximately $0.35 \mathrm{~ms}$. The inertia is set in free vibration about its new equilibrium point, $T_{\text {kin }}$. When the system torque decreases to $T_{\text {kin }}$, the relative velocity of the bushing and the shaft will be at its maximum. The relative velocity returns to zero to end one stick-slip cycle. The system torque is plotted as a function of the relative velocity for several stick-slip cycles in Figure 6.2. The two distinct phases of the cycle are the vertical line indicating the stick phase and the lobes corresponding to the system torque. Figure 6.3 is the phase plane representation of this behavior.

The behavior is different when the shaft velocity is increase to $\omega_{\text {shaft }}=10 \mathrm{rad} / \mathrm{s}$. Figure 6.4 shows the torque, displacement, and relative velocity time histories. After the slip at approximately $0.4 \mathrm{~ms}$, the system again responds to the step change in friction torque. The relative velocity of the shaft and bushing does not return to zero so the system remains in free vibration about $T_{\text {kin }}$. The potential energy of the system was less at $10 \mathrm{rad} / \mathrm{s}$ than at $1 \mathrm{rad} / \mathrm{s}$. At $1 \mathrm{rad} / \mathrm{s}$ the relative velocity plot in Figures 6.1 shows 

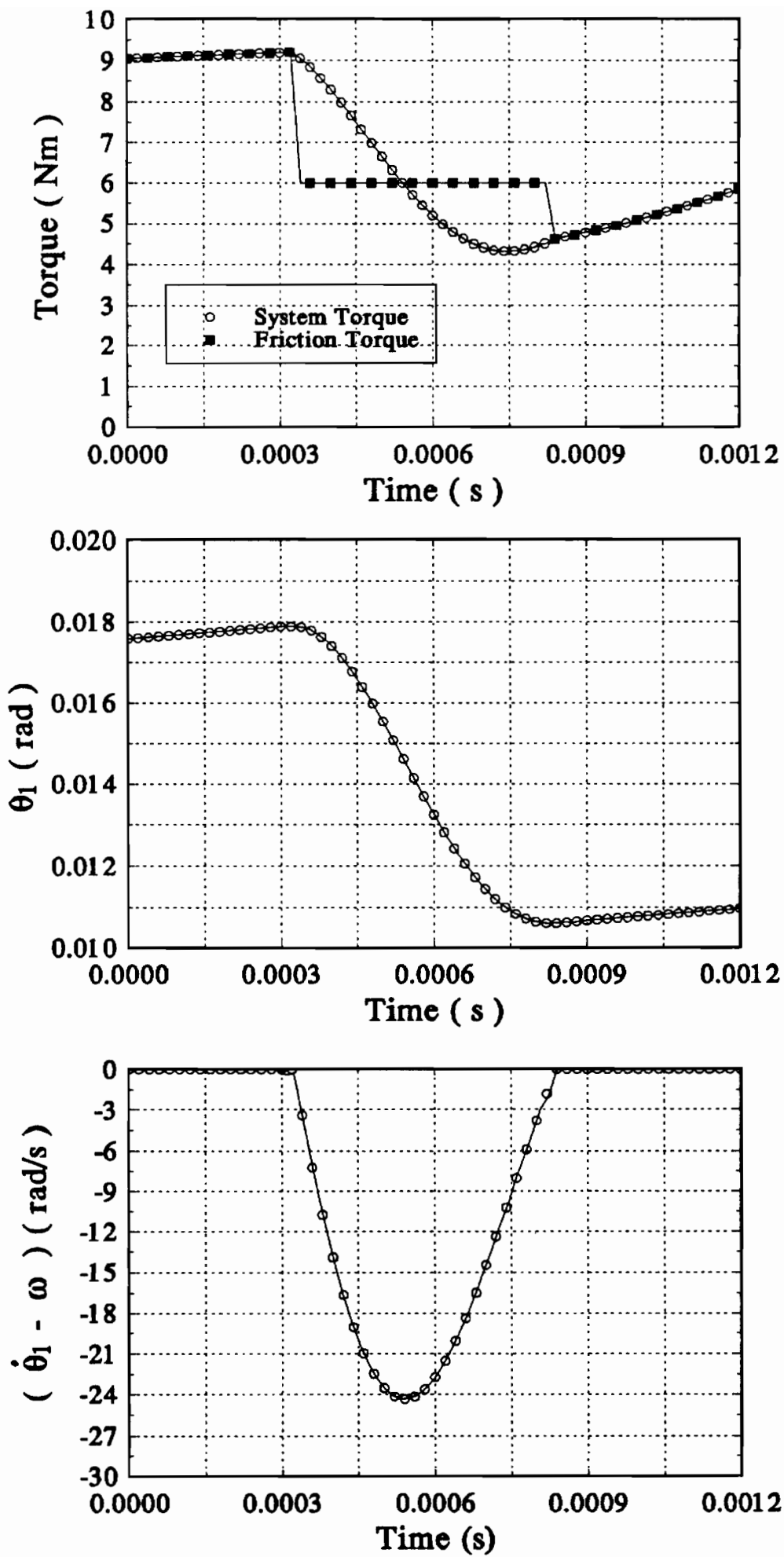

Figure 6.1 - Torque, displacement, and relative velocity during a slip event $\left(\omega_{\text {shaf }}=1 \mathrm{rad} / \mathrm{s}, k_{B}=2,214\right.$, and $\left.\delta=0.279 \mathrm{~mm}\right)$. 


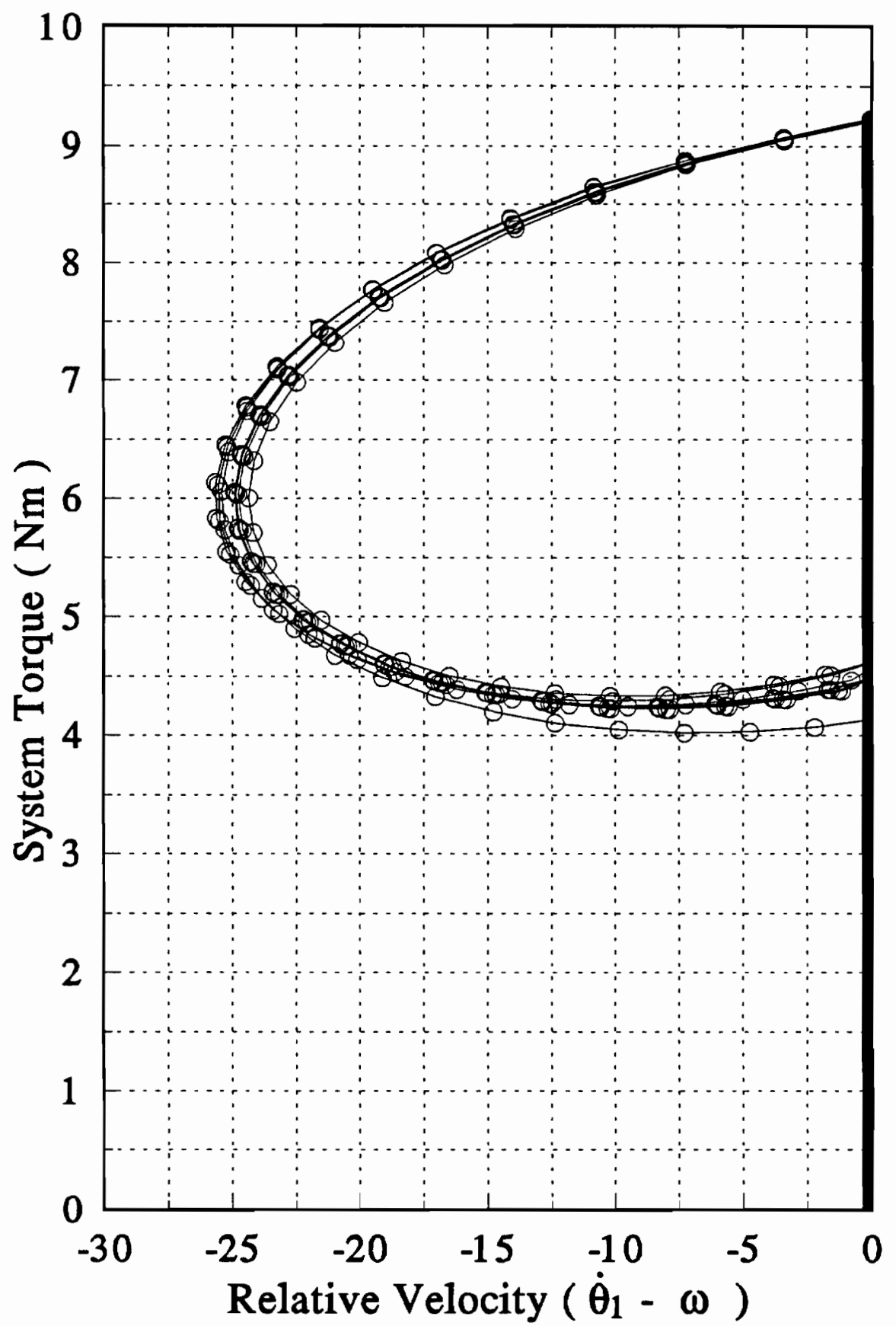

Figure $6.2-$ Torque/velocity relationship during successive stick-slip cycles.

Discussion

100 


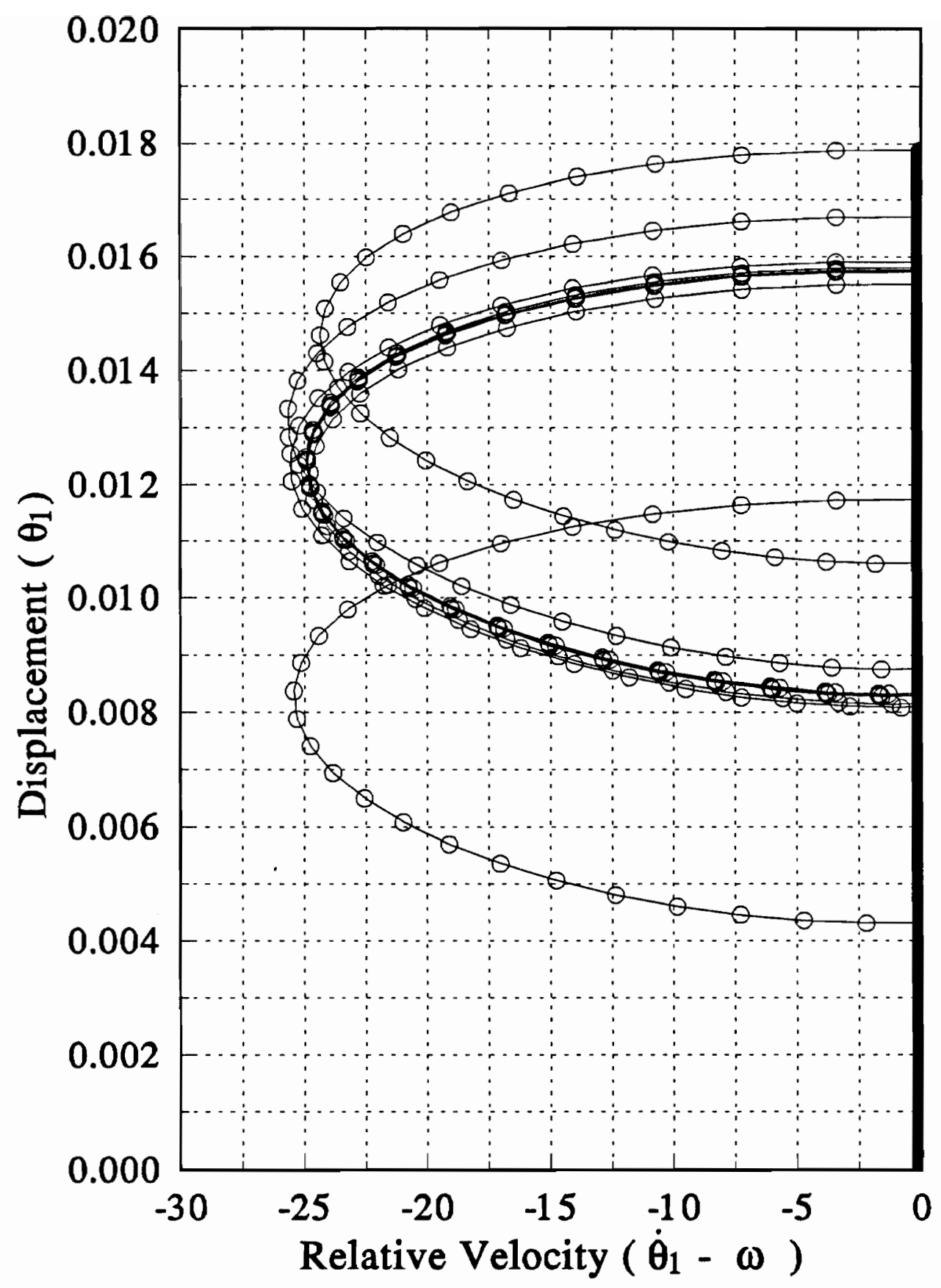

Figure 6.3 - Phase plane representation of stick-slip behavior. 

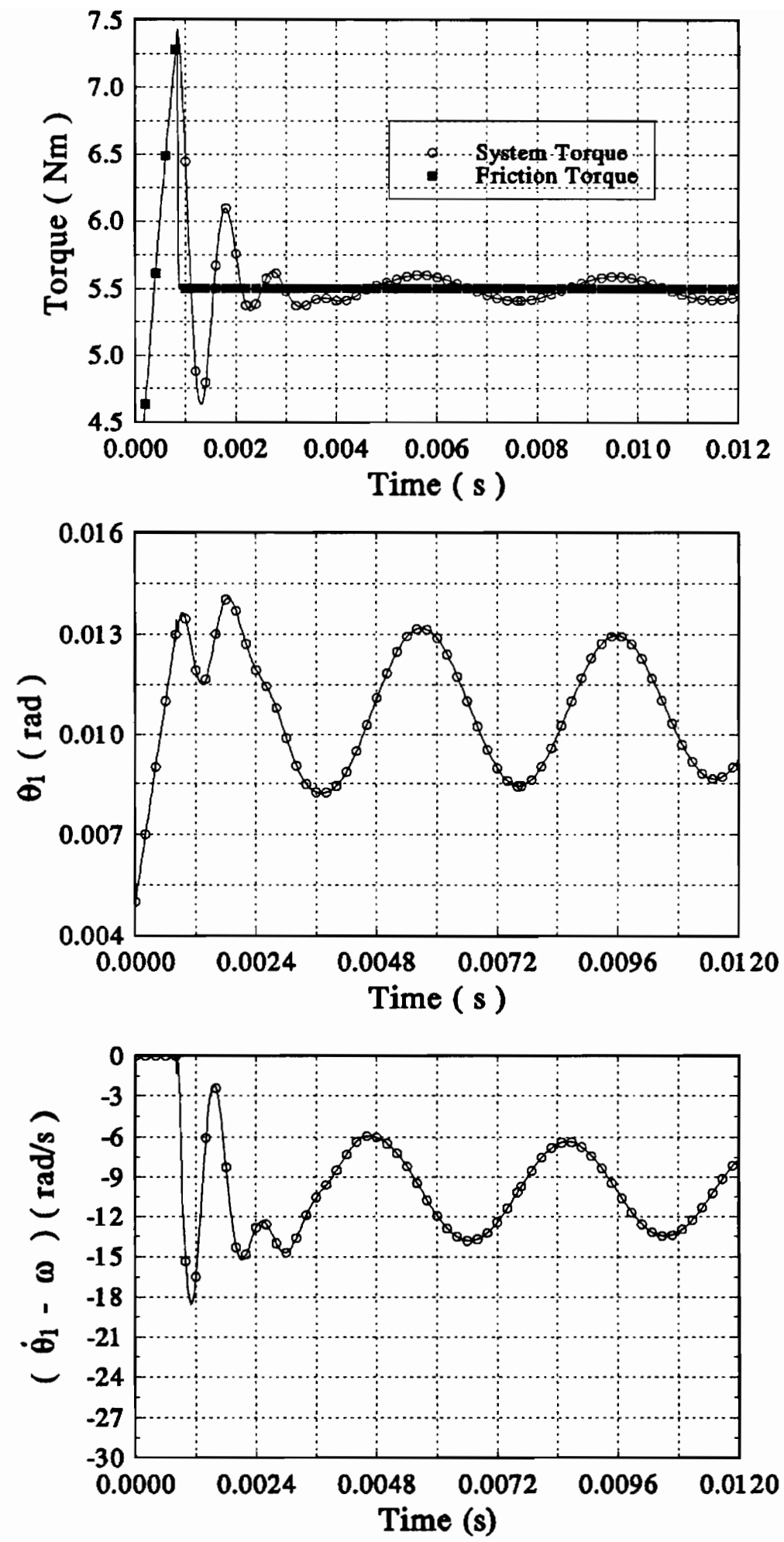

Figure 6.4 - Torque, displacement, and relative velocity at onset of harmonic oscillations $\left(\omega_{\text {shaft }}=10 \mathrm{rad} / \mathrm{s}, k_{B}=2,214\right.$, and $\delta=0.279 \mathrm{~mm}$ ). 
a peak at $24 \mathrm{rad} / \mathrm{s}$. In Figure 6.4 the maximum relative velocity attained was $18 \mathrm{rad} / \mathrm{s}$ indicating less potential energy was available to be converted to kinetic energy. Had the difference between $T_{\text {cris }}$ and $T_{k i n}$ been larger, the increased potential energy of the system would have allowed the system to regain the stick condition, ie., $\dot{\theta}_{I}=\omega$. Similarly, if the system stiffness were less, stick-slip would have resumed.

Figures 6.5 and 6.6 show the harmonic vibration behavior as indicated by the circular trace. Unlike the simulation at $1 \mathrm{rad} / \mathrm{s}$, the system cannot re-establish the zero relative velocity condition. For these operating conditions, however, the system torque spirals toward the equilibrium point at $T_{\text {kin }}$, where the system oscillation slowly decays in free vibration. Had the system damping been less or the difference in static and kinetic friction been greater, stick would have resumed.

Stick-slip occurred throughout the 1-10 rad/s shaft velocity range for the friction simulation of the largest interference fit, $\delta=0.381 \mathrm{~mm}$. This also is in agreement with the experimental transition velocities in Table 5.1. Figure 6.7 shows the system torque $\left[1 / 2 c_{E}\left(\dot{\theta}_{1}-\dot{\theta}_{2}\right)+k_{E}\left(\theta_{1}-\theta_{2}\right)\right]$, the displacement, $\theta_{1}$, and the relative angular velocity, $\left(\dot{\theta}_{1}-\omega\right)$. The 0.381 interference fit increases the energy of the system which almost doubles the relative velocity of the $0.279 \mathrm{~mm}$ fit simulation. Thus to achieve the transition for steady sliding, the shaft velocity must be greater as load increases so that the zero relative velocity condition is not met.

\subsubsection{Comparison of Actual and Simulated Behavior}

The time histories and autospectra of the simulated friction behavior and 


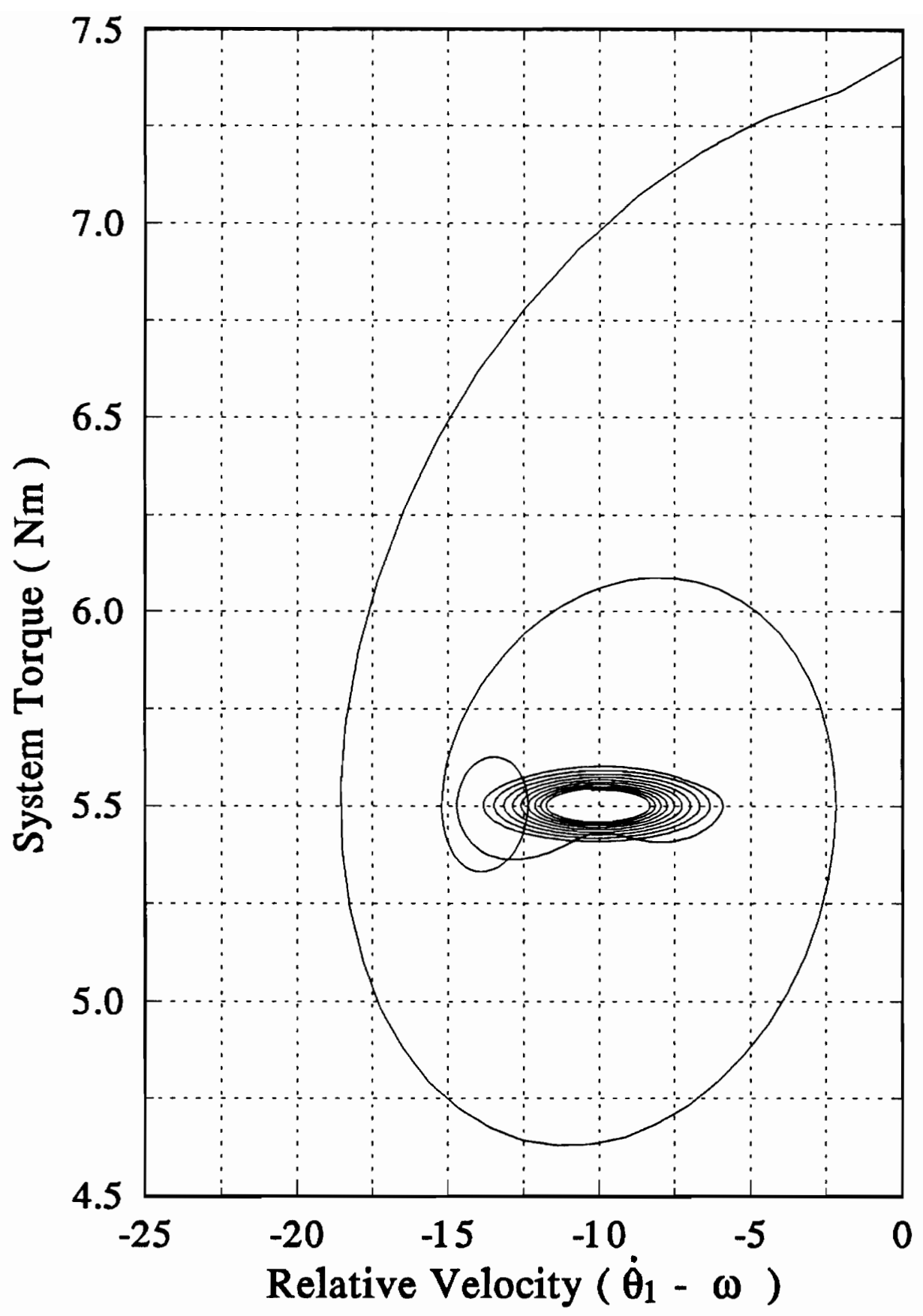

Figure $6.5-$ Torque/velocity relationship during harmonic oscillations. 


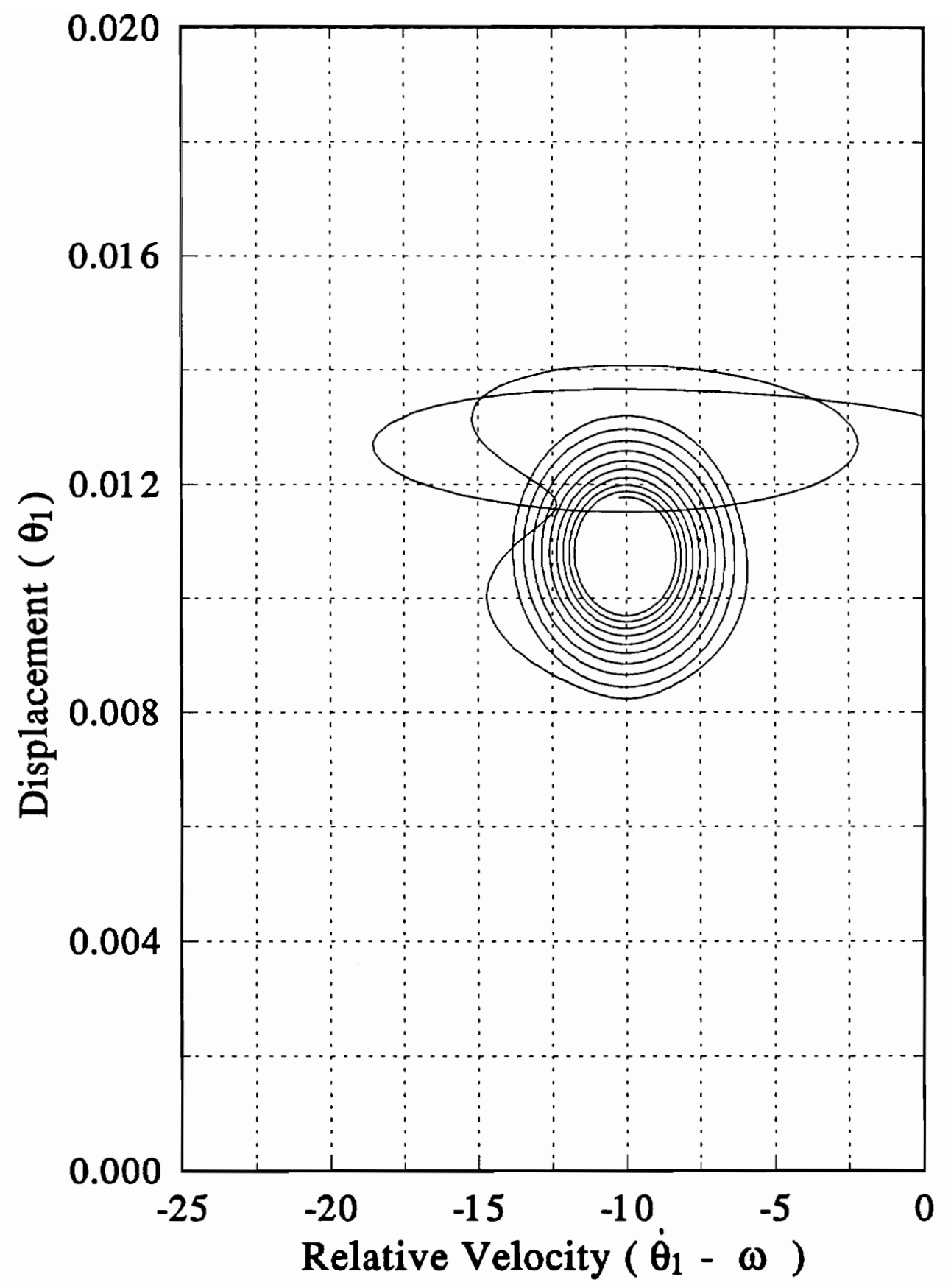

Figure 6.6 - Phase plane representation of harmonic oscillations. 

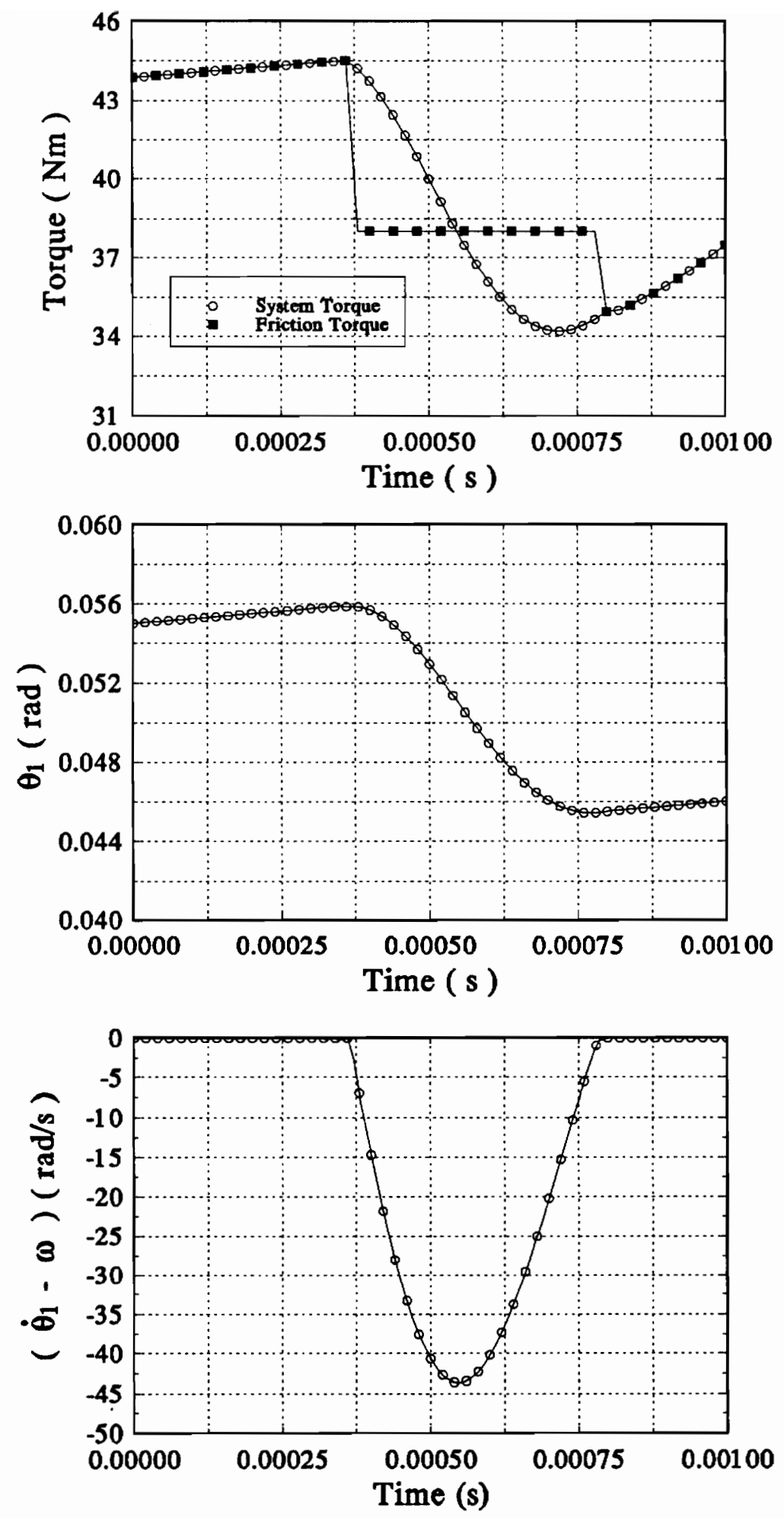

Figure $6.7-$ Torque, displacement, and relative velocity during a slip event $\left(\omega_{\text {shaft }}=1 \mathrm{rad} / \mathrm{s}, k_{B}=3,745\right.$, and $\left.\delta=0.381 \mathrm{~mm}\right)$. 
experimental data show similar behavioral trends. Figure 6.8 displays simulated and actual data for the operating conditions $\omega_{\text {shaft }}=1 \mathrm{rad} / \mathrm{s}, k_{B}=2,214$, and $\delta=0.279 \mathrm{~mm}$. Both time histories of stick-slip behavior exhibit superimposed frequency during the stick phase. The modelling assumptions require $\dot{\theta}_{1}=\omega_{\text {shaff }}$. Therefore the superimposed friction torque oscillation is caused by vibration of the clamp inertia. In Figure 6.8(a), the superimposed frequency is near the first natural frequency of the model. The autospectrum of the simulated behavior in Figure 6.8(b) and of the experimental data in Figure 6.8(d) also show similar trends. The decreasing amplitude of the harmonics are typical of the frequency spectrum of a pure sawtooth wave form. The third peak is greater in each case due to the proximity of the harmonic in relation to the first torsional mode of vibration.

Figure 6.9(a) and (b) are simulated friction torque and clamp acceleration. The bifurcated friction signal alternated between an equilibrium cycle with and without superimposed oscillations. This is due to the phase relationship between the two inertias. This phenomenon is observed in experiment through $\omega=2.5 \mathrm{rad} / \mathrm{s}$, with $k_{B}=2,214$ $\mathrm{Nm} / \mathrm{rad}$ and $\delta=0.279 \mathrm{~mm}$. The predominant frequency in the acceleration signal is the fundamental mode of the system model. Aside from the noise and higher mode frequencies in the beam acceleration in Figure 6.9(d), there is good agreement with simulated acceleration behavior.

Using system parameters from actual tests, the model shows the relative effects of input shaft velocity, system stiffness, and load. One parameter which cannot be varied in experiment was the system damping. The effect of varying the damping was 

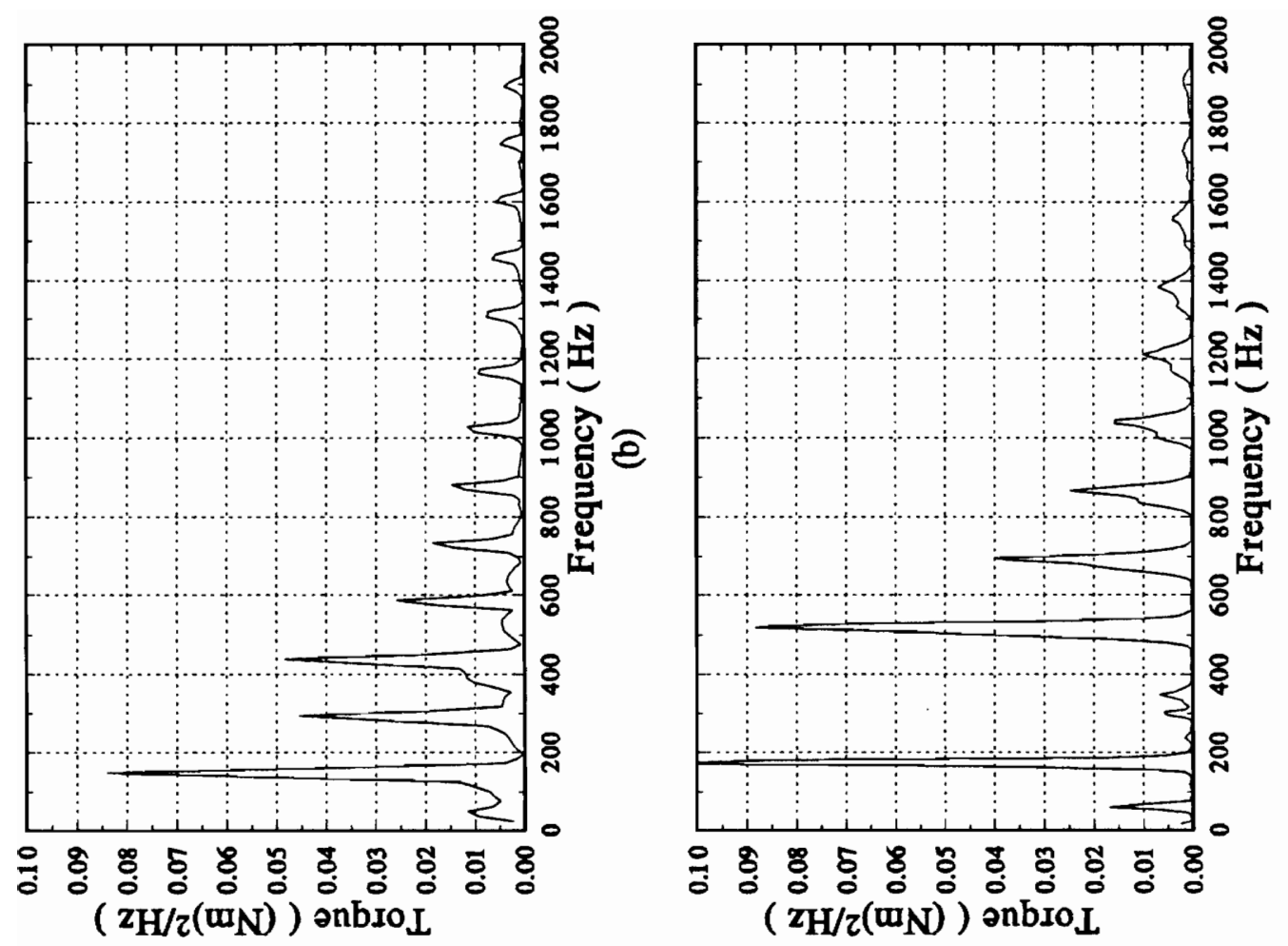

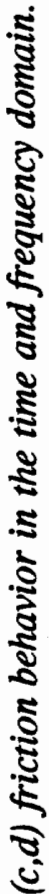
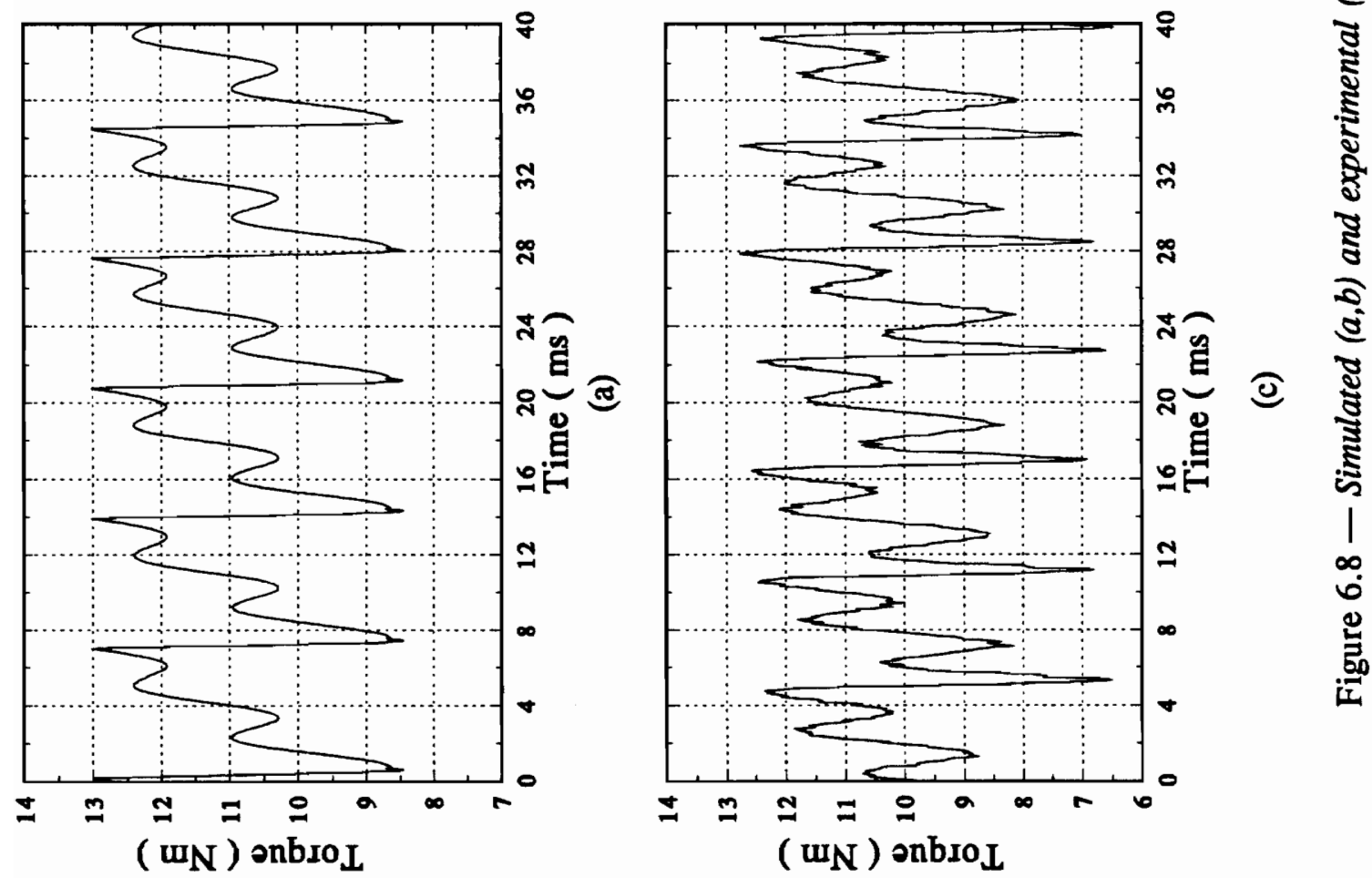

Discussion 

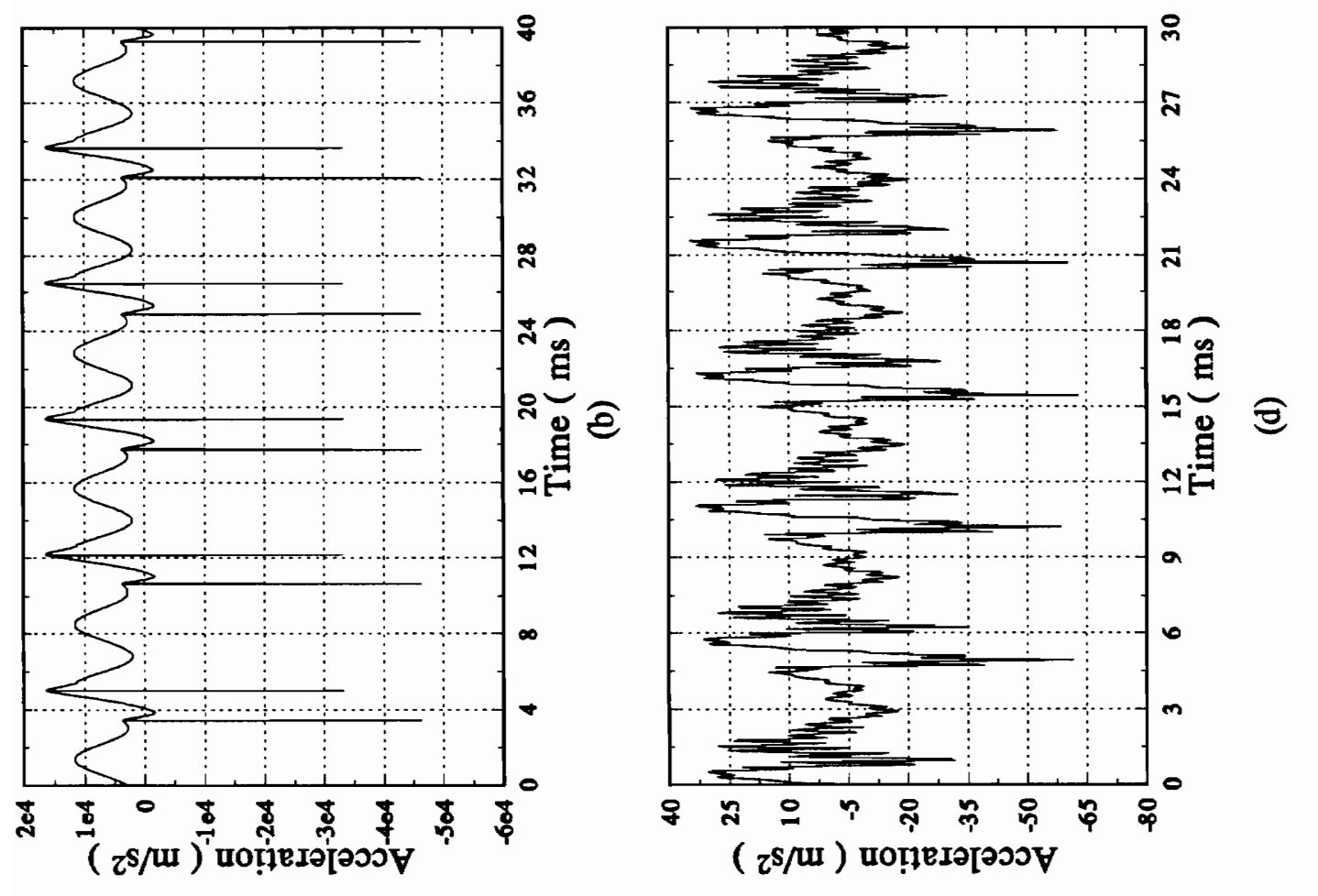

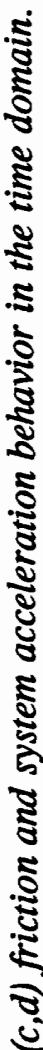
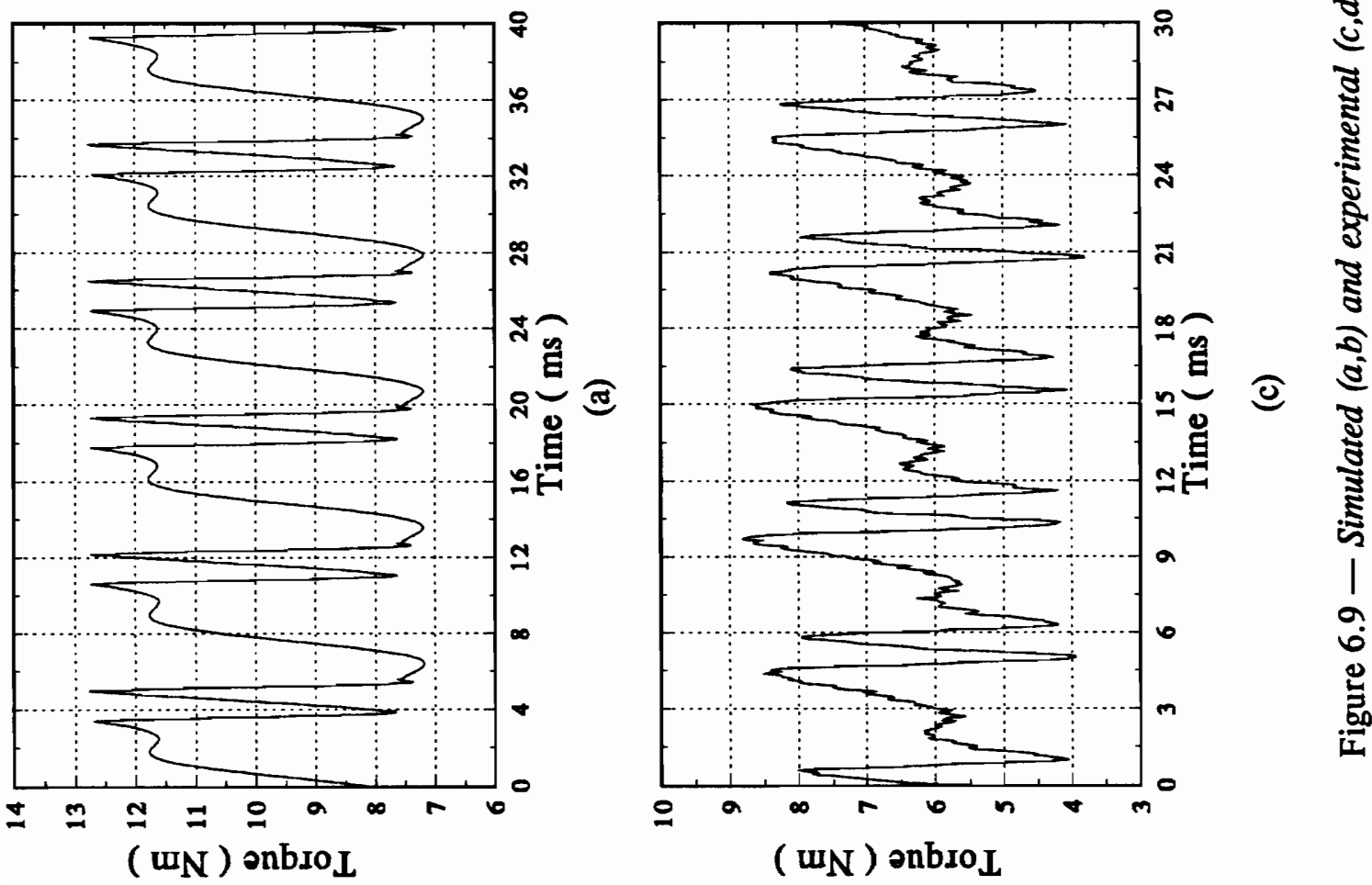
investigated using the model. The friction was simulated for the conditions $\omega_{\text {shaft }}=7.5$ $\mathrm{rad} / \mathrm{s}, k_{B}=3,745 \mathrm{Nm} / \mathrm{rad}$, and $\delta=0.279 \mathrm{~mm}$. Stick-slip was not observed at these operating conditions in experiment or in simulation. The damping coefficient, $c_{E}$, was decreased to $0.05 \mathrm{kgm}^{2} / \mathrm{rad} / \mathrm{s}$ (less than one half of the experimental value). Stick-slip was induced with this damping level. Figure 6.10 shows this behavior. At $c_{E}=0.1$ $\mathrm{kgm}^{2} / \mathrm{rad} / \mathrm{s}$ harmonic oscillations occurred. A critical damping coefficient must exist below which the trajectory would intercept the driven velocity causing stick and above which harmonic oscillations would occur. The critical damping for these operating conditions was found iteratively to be $0.7599 \mathrm{kgm}^{2} / \mathrm{rad} / \mathrm{s}$. The phase plane plot shows the velocity falls between the stick-slip and oscillating case.

It is clear through observation of Figure 6.10 that the occurrence of stick-slip is dependent simultaneously on the driven velocity of the shaft and the displacement of the elastomer inertia from its equilibrium position. When the damping coefficient equalled $0.76 \mathrm{kgm}^{2} / \mathrm{rad} / \mathrm{s}$, the trajectory intercepts $\dot{\theta}_{1}=7.5 \mathrm{rad} / \mathrm{s}$ tangentially. Increasing the driven velocity, decreasing the potential energy of the system, and/or increasing the system damping will cause the motion to follow a trajectory that will spiral towards an equilibrium point or a limit cycle. Similarly, for the same damping condition, the point at which the motion reaches its maximum displacement from the equilibrium position is the minimum displacement required for stick-slip oscillation. Increasing the system stiffness will inhibit stick-slip behavior. Conversely, decreasing it will allow greater displacement from the equilibrium position causing the motion to follow a trajectory that will intercept the $7.5 \mathrm{rad} / \mathrm{s}$ velocity causing the stick phase to resume. Similar 


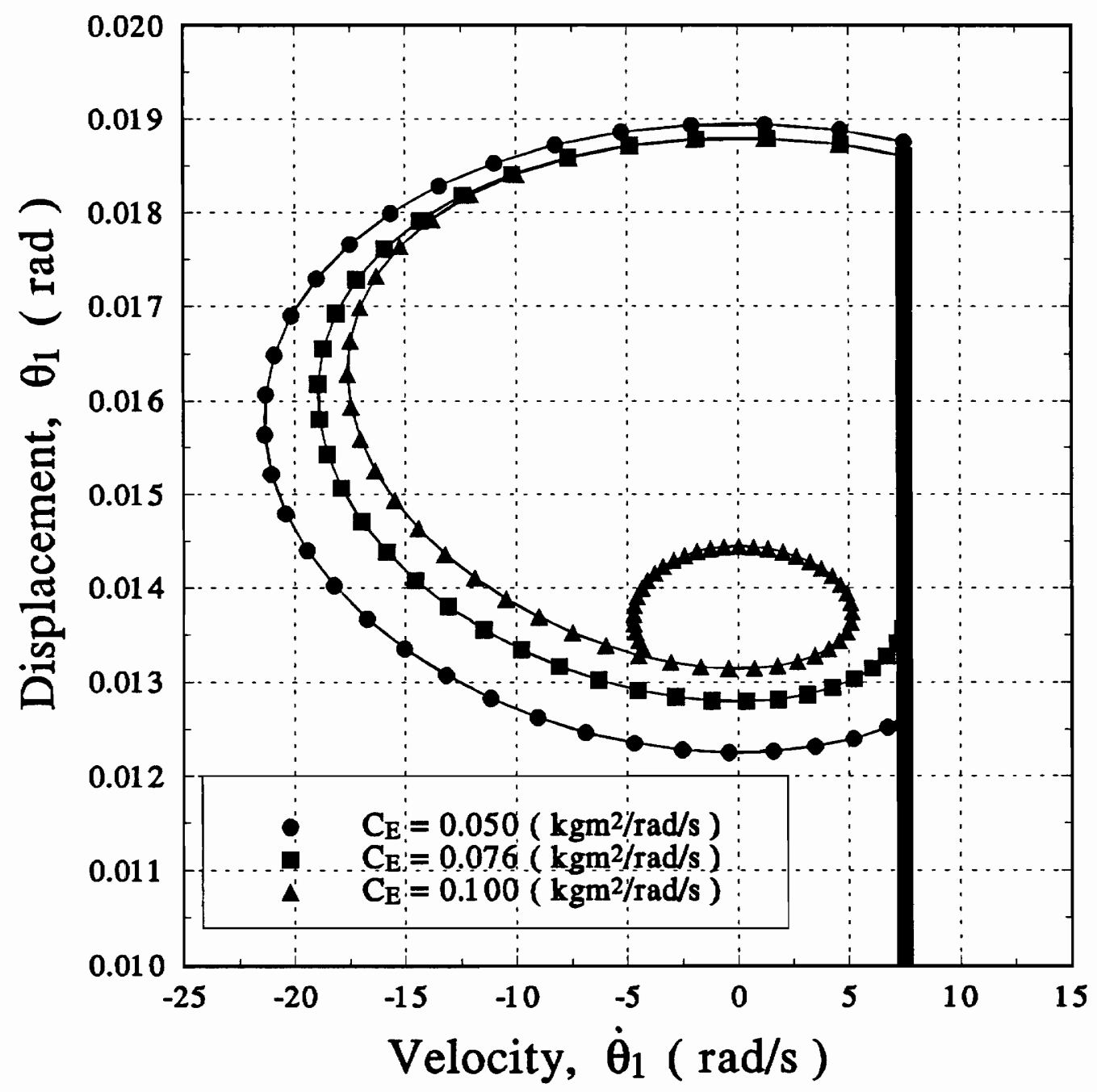

Figure $6.10-$ Effect of damping on friction induced vibration behavior. 
conclusions were drawn by Brockley, et al. [10] when they developed a one degree-offreedom stick-slip model.

\subsubsection{Comments Regarding the Validity of the Model}

Several explanations regarding stick-slip have been formulated. The difference between static and kinetic friction coefficients is accepted as one of the requirements for this type of friction behavior. Further, investigations have shown from nonlinear vibration theory that velocity dependent friction is a contributing and necessary factor. This simulation does not provide dynamic friction during the slip phase. In Figure 6.7 $\left(\dot{\theta}_{1}-\omega_{\text {shaff }}\right)$ reaches its maximum at $45 \mathrm{rad} / \mathrm{s}$ which translates to a linear sliding speed range of $67 \mathrm{~cm} / \mathrm{s}$. One would expect a change in the friction coefficient of the elastomer over this range. However, referring to Figure 5.3, the friction torque during steady sliding assumes the mean friction torque level. There for $T_{\text {kin }}=$ constant seems appropriate for this model.

The model has its limitations. The friction and system behavior has constant shaft velocity input. The displacement of the elastomer monotonically increases with time implying a constant contact area is maintained at the interface. This is not found to be true in experiment. The critical shear load may be constant, but the contact area must change since $T_{\text {crit }}$ varies slightly throughout the experiment. The final limitation of the model is the modes of vibration. During the stick phase, there is only one mode of vibration. This is not a bad assumption if one considers translational modes of beam 
vibration are minimized by the torsional.input and the first torsional mode dominates the others. These things aside, the model's friction behavior shows the relative effects of system stiffness, velocity, and interference fit on friction induced vibration. The phase plane plots allow the user to determine system damping requirements for stick-slip, harmonic oscillations, or steady sliding for given operating conditions. The system model correlates well with experimental trends and allows the user to predict with confidence the frictional behavior for the fluorocarbon bushing. 


\section{Conclusions}

1. Vibration results from stick-slip and harmonic oscillations at the stabilizer bar/bushing interface. Energy is transferred from the excited beam system back to the interface to influence the frequency spectra of the friction torque and the corresponding acoustic output.

2. Three frictional regimes observed in this investigation were stick-slip oscillation, quasi-harmonic oscillation, and steady sliding. The sliding velocity, pressure, and the stiffness of the supporting structure are factors that contribute to the frictional behavior of the sliding pair.

3. Increasing the velocity and system stiffness increases the stick-slip frequency which does not exceed the first torsional vibration mode of the system. Altering the system stiffness does not alter the amplitude of vibration, but the frequency content of the friction induced oscillation is affected considerably.

4. Although the torsional stiffness and amplitude of frictional oscillation of the elastomer increase with increased pressure, the frequency of oscillation decreases. At sufficiently high pressure, relative motion does not occur.

5. The elastomer characteristics including friction/velocity relationships, friction mechanisms, and mechanical properties interact with system dynamics to influence elastomeric friction behavior. The relative contribution of friction by adhesion and deformation determined the stability of sliding. The fluorocarbon and natural rubber materials exhibiting frictional instabilities were able to transmit sufficient energy to the system by adhesion. PDMS did not have a difference between static and kinetic friction 
and had a positive of friction/velocity relationship which are considered requirements for steady sliding.

6. Several types of noise were generated and the mechanical and acoustical response associated with each were examined. The type of frictional instability influenced the character of the noise as would be expected. The frequency response of the apparatus to the magnitude of oscillation was also a factor. The relative amplitude of stick-slip vibration and the energy density of the frequency spectrum dictated which modes of the apparatus were excited and the character of the sound radiated.

7. The simulated sliding behavior makes it possible to determine the displacement, velocity, damping, stiffness, and inertia required for stable sliding for the apparatus used in this investigation. The model may be extended or modified to simulate the sliding behavior of other systems as well. 


\section{Recommendations}

1. The sliding speed dependence of friction must be analyzed and extended over a broader range of relative sliding velocities. This may be accomplished by adding transducers to the apparatus which measure the displacement, velocity, and acceleration of the elastomer at the interface. The solution of the instantaneous friction velocity relationship would be found by substituting these parameters into the equations of motion of the system.

2. A suitable method of determining experimentally the normal contact pressure and real area of contact must be developed. The change in interference fit does not provide a viable means of comparison between materials with different elastic moduli. Pressure sensitive film is one possible alternative. Another solution is to use optic fibers such that the signal is attenuated under applied pressure. One final method to determine the interfacial pressure is to mount semi-conductor strain gages on the inside and edge of the shaft and find the radial and circumferential stress imposed by clamping the bushing with a certain interference fit. The strains could then be substituted into the well known Lame' solution of an internally and externally pressurized cylinder to solve for the interfacial pressure.

3. The experimental apparatus has many acoustic transmission paths. Mounting the apparatus on a marble or concrete slab would minimize the surface area of acoustic emissions which would produce better correlation between friction, system, and sound pressure measurements

4. Sound power as a result of friction induced vibration should be measured in different octave bands. This might be accomplished by placing a hemispherical array of sound 
intensity probes about the test rig. Then comparisons of sound power could be drawn regarding operating conditions and the experimental materials.

5. Environmental factors are an important aspect of elastomeric friction. Experiments involving different surface coatings of the shaft, dirt, oil, water, and temperature would be essential to final stabilizer bushing design.

6. New elastomers should be modified and tested. These materials should possess excellent compressive and tear strength, low adhesive strength, and resistance to the end use environment. The frequency dependence of the damping and the complex modulus would be important factors in finding a suitable elastomeric material.

7. The culmination of this work should be toward developing a multi degree-of-freedom model of the actual suspension system of the car. Structural modes and damping of the stabilizer bar and the support structure, shaft displacement and velocity, and the normal load should be experimentally determined. These parameters and the properties of potential elastomeric materials may then be incorporated to examine friction and acoustic behavior analytically. 


\section{References}

[1] Tabor, David, "Friction, Adhesion, and Boundary Lubrication of Polymers," Advances in Polymer Friction and Wear, Plenum Press, 1972, pp. 5-31.

[2] Roberts, A. D., "Theories of Dry Rubber Friction." Tribology International, Vol.9, N0. 2, April 1976, pp. 75-81.

[3] Greenwood, J.A, and Tabor, David, "The Friction of Hard Sliders on Lubricated Rubber: the importance of deformation losses," Proc. Phys. Soc., 71, 1958, pp. 989-1001.

[4] Schallamach, A., "How Does Rubber Slide?" Wear, 17, 1971, pp. 301-312.

[5] Grosch, K.A., "The relation between the friction and visco-elastic properties of rubber," Proceedings of the Royal Society (London), Series A, Vol. 274, 1963, pp. 21-39,

[6] Moore, D.F., and Geyer, W., "A Review of Adhesion Theories For Elastomers," Wear, Vol. 22, 1972, pp. 113-141.

[7] Ludema, K.C., "Friction," CRC Handbook of Lubrication, Vol II, Ed. E.R. Booser, CRC Press, Inc., Boca Raton, FL, 1984, pp. 31-48.

[8] Bowden F.P. and Leben, L., "The Nature of Sliding and the Analysis of Friction," Proc. Roy. Soc. London, A169, 1939, pp. 371-391.

[9] Rabinowicz, E., Friction and Wear of Materials, John Wiley and Sons, Inc., New York, 1965. pp. 152-153.

[10] Brockley, C. A., Cameron R., and Potter, A. F., "Friction-Induced Vibration," ASME Journal of Lubrication Technology, Vol. 89, April 1967, pp. 101-108.

[11] Brockley, C. A., and Ko, P. O., "Quasi-Harmonic Friction Induced Vibration," ASME Journal of Lubrication Technology, October 1970 Vol. 92, pp. 550-556.

[12] Ko, P. L., and Brockley, C. A., "The Measurement of Friction and FrictionInduced Vibration," ASME Journal of Lubrication Technology, Vol. 92, Oct. 1970, pp. 543-549. 
[13] Aronov, V., D'Souza, A. F., Kalpakjian, S., and Shareef, I., "Interactions Among Friction, Wear, and System Stiffness-Part 2: Vibrations Induced by Dry Friction," ASME Journal of Lubrication Technology, Vol. 106, January 1984, pp. 59-64.

[14] Aronov, V., D'Souza, A. F., Kalpakjian, S., and Shareef, I.,"Experimental Investigation of the Effect of System Rigidity on Wear and Friction-Induced Vibrations," ASME Journal of Lubrication Technology, Vol. 105, April 1983, pp. 206-211.

[15] Aronov, V., D'Souza, A. F., Kalpakjian, S., and Shareef, I.,"Interactions Among Friction, Wear, and System Stiffness-Part 1: Effect of Normal Load and System Stiffness," ASME Journal of Lubrication Technology, Vol. 106, January 1984, pp. 54-58.

[16] Kiryu, K., Yanai, T., Matsumoto, S., and Koga, T., "An Analysis of "Ringing" Phenomena on a Water Pump Mechanical Seal," ASLE Transactions, Vol. 28, No. 2, 1985, pp. 261-267.

[17] Kiryu, K., Yanai, T., Matsumoto, S., and Koga, T., "An Analysis of "Ringing" Phenomena on a Water Pump Mechanical Seal (Part III)," ASLE Transactions, Vol. 31, No. 2, 1987, pp. 269-275.

[18] Bhushan, B., "Stick-slip Induced Noise Generation in Water Compliant Rubber Bearings," ASME Journal of Lubrication Technology, Vol. 102, April 1980, pp. 201-212.

[19] Kato S. and Matsubayachi T., "On the Dynamic Behavior of Machine Tool Slideways," Bulletin of the JSME, Vol. 13(55), 1970, pp. 170-179.

[20] Kiryu, K., Yanai, T., Matsumoto, S., and Koga, T., "An Analysis of "Ringing" Phenomena on a Water Pump Mechanical Seal (Part II)," ASLE Transactions, Vol. 31, No. 1, 1986, pp. 25-34.

[21] Schwartz H.W., Hays, D. Jr., and Tarter, J.H., "A systematic Approach to the Analysis of Brake Noise," Surface Vehicle Noise and Vibration Conference Proceedings, P-161, Society of Automotive Engineers, Inc., May 1985, pp. 267275.

[22] Earles, S.W.E, and Soar, G.B., "A Vibrational Analysis of a Pin-Disc System with Particular Reference to Squeal Noise in Disc Brakes," Stress, Vibration, and Noise Analysis in Vehicles, Halsted Press, 1975, pp. 237-251. 
[23] Othman, M. O., Elkholy, A. H., and Seireg, A. A., "Experimental Investigation of Frictional Noise and Surface-roughness Characteristics," Experimental Mechanics, Vol. 30(4), December 1990, pp. 328-331.

[24] Symmons, G.R. and McNulty, G.J., "Acoustic Output from Stick-slip Friction," Wear Vol. 113, 1986, pp. 79-82.

[25] Brydson, J.A., Rubbery Materials and Their Compounds, Elsevier Science Publishing Co, New York, NY, 1988, p. 261.

[26] Thomson, W.T., Theory of Vibration with Applications, Prentice Hall, Englewood Cliffs, NJ, 1988, pp. 108-110.

[27] Sweet, G.C., in Developments in Rubber Technology-1, ed. Whelan, A. and Lee, K.S., Applied Science, London, 1979, Chapter 2.

[28] Vick, B., Private conversation on 26 February, 1992.

[29] Incropera, F.P. and DeWitt, D.P., Fundamentals of Heat and Mass Transfer, John Wiley \& Sons, 1985, pp. 202-204.

[30] Bendat, J.S. and Piersol, A.G., Random Data: Analysis and Measurement Procedures, 2nd Edition, John Wiley \& Sons, 1986, pp. 391-409. 


\section{Appendix A. Theoretical Solution of Shaft Natural Frequencies}

The torsional vibration of the primary and secondary shaft is derived to bound the range of resonant frequencies and ensure the range is higher than the frequency of frictional vibration. Referring to the figure below the equation of motion is derived.

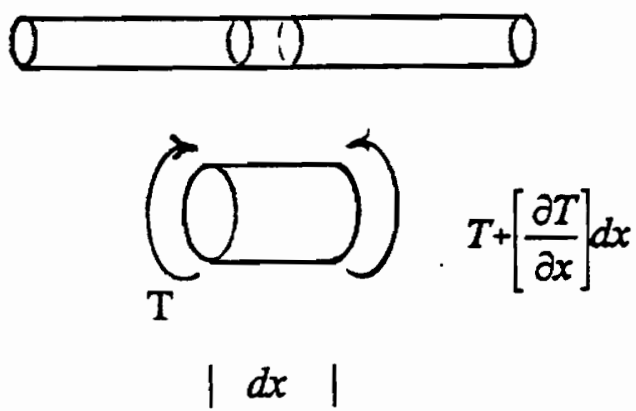

Figure A1 - Torque acting on a rod element $d x$.

The angle of twist in $d x$ of a rod due to torque, $T$, where $I_{p} G$ is the torsional stiffness given by $I_{p}$ (polar moment of inertia of $A_{\text {cross }}$ and shear modulus G)

$$
d \theta-\frac{T}{I_{p} G} d x
$$

Assuming net torque on two faces of the element are $T$ and $T+\left[\frac{\partial T}{\partial x}\right] d x$, the net torque becomes

$$
\frac{\partial T}{\partial x} d x-I_{p} G \frac{\partial^{2} \theta}{\partial t^{2}} d x
$$


Equating torque to the product of mass moment of inertia and angular acceleration yields

$$
\begin{aligned}
\rho I_{p} d x \frac{\partial^{2} \theta}{\partial t^{2}} & -I_{p} G \frac{\partial^{2} \theta}{\partial x^{2}} d x \\
\frac{\partial^{2} \theta}{\partial t^{2}} & =\left[\frac{G}{\rho}\right] \frac{\partial^{2} \theta}{\partial x^{2}}
\end{aligned}
$$

Separation of variables was used to solve the partial differential equation of motion assuming a solution of the form

$$
\theta-Y(x) Z(t)
$$

Substitution gives

$$
\frac{1}{Y} \frac{\partial^{2} Y}{\partial x^{2}}=\frac{G}{\rho} \frac{1}{Z} \frac{\partial^{2} Z}{\partial t^{2}}
$$

Since each side of the equation independent of the variable of the other, each side must be equal to a constant. Assume each side is equal to $-\frac{\omega^{2} \rho}{G}$ yielding

$$
\begin{array}{r}
\frac{d^{2} Y}{d x^{2}}+\omega^{2} \frac{\rho}{G} Y=0 \\
\frac{d^{2} Z}{d t^{2}}+\omega^{2} Z=0
\end{array}
$$

with general solutions:

$$
\begin{aligned}
& Y=A \sin \omega \sqrt{\frac{\rho}{G}} x+B \cos \omega \sqrt{\frac{\rho}{G}} x \\
& Z-C \sin \omega t+D \cos \omega t
\end{aligned}
$$


The solution takes the form

$$
\theta-\left[A \sin \omega \sqrt{\frac{\rho}{G}} x+B \cos \omega \sqrt{\frac{\rho}{G}} x\right][C \sin \omega t+D \cos \omega t]
$$

where $A, B, C$, and $D$ depend on boundary and initial conditions.

With this solution, the expressions for the natural frequencies of the shaft in torsional oscillation with fixed-free and free-free boundary conditions are derived. The natural frequencies for each of these boundary conditions will define the upper and limits the actual natural frequencies.

For the fixed-free case, assume the primary shaft is fixed at one end and the secondary shaft can be represented as a mass of moment of inertia $J_{0}$. The shaft experiences torque $T_{\text {shaft }}$ and the inertial torque $T_{J o}$.

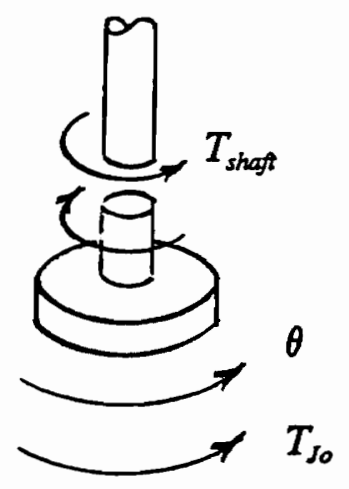

Figure A2 - Shaft torque $T_{\text {shaft }}=G I_{p}(d \theta / d x)_{x=l}$ and inertia torque $T_{J o}=-J_{o}\left(\partial^{2} \theta / \partial x^{2}\right)_{x=l}$

Applying boundary condition (1) to equation (A8):

$$
x=0 ; \theta-0
$$


yields

$$
0=\left[A \sin \omega \sqrt{\frac{\rho}{G}} 0+B \cos \omega \sqrt{\frac{\rho}{G}} 0\right][C \sin \omega t+D \cos \omega t]
$$

Equating the shaft and inertial torque and applying boundary condition (2) gives

$$
x-l ; \quad G I_{p}\left[\frac{\partial \theta}{\partial x}\right]_{x-l}-J_{0} \omega^{2}(\theta)_{x-l}
$$

Substituting the following equations into equation (A11)

$$
\begin{gathered}
\theta-\left[A \sin \omega \sqrt{\frac{\rho}{G}} \eta[C \sin \omega t+D \cos \omega t]\right. \\
\frac{\partial \theta}{\partial x}-\left[\omega \sqrt{\frac{\rho}{G}} A \cos \omega \sqrt{\frac{\rho}{G}} l[C \sin \omega t+D \cos \omega t]\right.
\end{gathered}
$$

and rearranging yields

$$
\begin{aligned}
\tan \omega l \sqrt{\frac{\rho}{G}} & -\frac{G I_{p}}{\omega J_{0}} \sqrt{\frac{\rho}{G}}-\frac{I_{p}}{\omega J_{0}} \sqrt{\rho G}-\frac{\rho I_{p} l}{\omega J_{0} l} \sqrt{\frac{G}{\rho}} \\
& =\frac{J_{\text {shat }}}{J_{0} \omega l} \sqrt{\frac{G}{\rho}}
\end{aligned}
$$

Therefore

$$
\omega-\frac{\beta}{l} \sqrt{\frac{G}{\rho}}
$$

where

$$
\beta \tan \beta=\frac{J_{\text {shaft }}}{J_{0}} ; \quad \beta=\omega l \sqrt{\frac{\rho}{G}}
$$


For the free-free vibration case, the stress at the ends must be equal to zero, ie.

$$
\begin{aligned}
& {\left[\frac{\partial \theta}{\partial x}\right]-0 \text {. Boundary condition (1) requires }} \\
& \qquad x-0 ; \frac{\partial \theta}{\partial x}=0 \\
& \frac{\partial \theta}{\partial x}-\left[\omega \sqrt{\frac{\rho}{G}} A \cos \omega \sqrt{\frac{\rho}{G}} x-\omega \sqrt{\frac{\rho}{G}} B \sin \omega \sqrt{\frac{\rho}{G}} x\right][C \sin \omega t+D \cos \omega t]
\end{aligned}
$$

thus yielding

$$
0=\omega \sqrt{\frac{\rho}{G}}[A \cos 0-B \sin 0][C \sin \omega t+D \cos \omega t]
$$

Applying boundary condition (2):

$$
\begin{gathered}
x=l ; \frac{\partial \theta}{\partial x}=0 \\
0-\left[-\omega \sqrt{\frac{\rho}{G}} B \sin \omega \sqrt{\frac{\rho}{G}} l\right][C \sin \omega t+D \cos \omega t]
\end{gathered}
$$

The result is

$$
\sin \omega l \sqrt{\frac{\rho}{G}}=0
$$

which is satisfied by angles

$$
\begin{gathered}
\omega l \sqrt{\frac{\rho}{G}}-0, \pi, 2 \pi, \ldots, n \pi \\
\therefore \quad \omega-\frac{n \pi}{l} \sqrt{\frac{G}{\rho}}
\end{gathered}
$$




\section{Appendix B. Calibration of Radial Potentiometer}

The radial potentiometer is a ten-turn, $5 \mathrm{~K} \Omega$ linear measuring device. The transducer was only calibrated from 0 to $\pi$ rad to ensure linearity over the maximum angular displacement of the torsional apparatus. Calibration data is listed in Table B1. An input voltage of $2 \pi$ volts was chosen to provide a gain of approximately $10 \mathrm{rad} / \mathrm{volt}$. Linear regression was used to determine the gain of the transducer. The following equations were solved simultaneously to determine the linear relationship.

$$
\begin{gathered}
n C_{0}+\sum \theta_{i}-\sum V_{i} \\
C_{0} \sum \theta_{i}+C_{1} \sum \theta_{i}^{2}-\sum V_{i} \theta_{i}
\end{gathered}
$$

The adequacy of the regression analysis was evaluated by calculating the correlation coefficient given by

$$
I=\frac{n \sum \theta_{1} V_{i}-\sum \theta_{1} \sum V_{i}}{\left[n \sum \theta_{i}^{2}-\left(\sum \theta_{1}\right)^{2}\right]^{\frac{1}{2}}\left[n \sum V_{i}^{2}-\left(\sum V_{i}\right)^{2}\right]^{\frac{1}{2}}}
$$

Solving the equations yielded the following linear relationship.

$$
V=-0.0007+0.101 \theta
$$

with

$$
r=0.9999
$$

The result is a gain of $9.9 \mathrm{rad} / \mathrm{volt}$. The calibration curve is shown in Figure B1 with the data points used to determine the angular displacement/voltage relationship. 
Table B1. Calibration Data for the radial potentiometer.

\begin{tabular}{||c|c||}
\hline $\begin{array}{c}\text { Displacement } \\
\text { [rad] }\end{array}$ & $\begin{array}{l}\text { Voltage } \\
\text { [volts] }\end{array}$ \\
\hline$\pi / 6$ & 0.0020 \\
\hline$\pi / 4$ & 0.0511 \\
\hline$\pi / 3$ & 0.0777 \\
\hline$\pi / 2$ & 0.1035 \\
\hline $2 \pi / 3$ & 0.1581 \\
\hline $3 \pi / 4$ & 0.2140 \\
\hline $5 \pi / 6$ & 0.2410 \\
\hline$\pi$ & 0.3161 \\
\hline
\end{tabular}




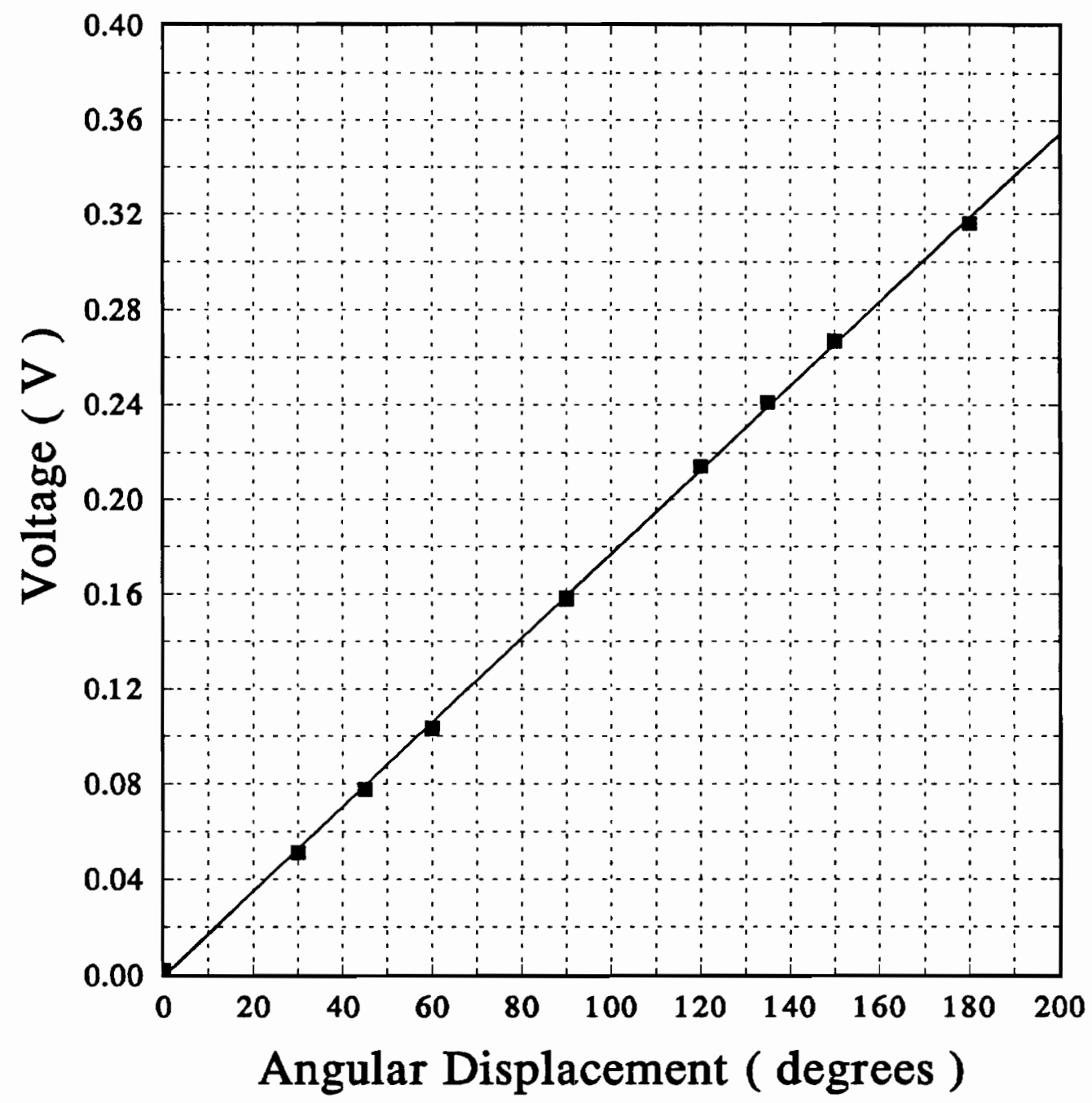

Figure B1 - Calibration Curve of the radial potentiometer. 


\section{Appendix C. Calibration of Torque Cell}

The torque cell consisted of four 45-degree strain gages mounted on the primary shaft. The cell was loaded in $5 \mathrm{Nm}$ increments over a $\pm 40 \mathrm{Nm}$ test range. The data for torque in the positive and negative sense and the corresponding voltages are tabulated in Table C1. Linear regression was used to determine the relationship between applied torque and output voltage. The following equations were solved simultaneously to determine the linear relationship.

$$
\begin{gathered}
n C_{0}+C_{1} \sum T_{i}-\sum V_{i} \\
C_{0} \sum T_{i}+C_{1} \sum T_{i}^{2}=\sum V_{i} T_{i}
\end{gathered}
$$

The adequacy of the regression analysis was evaluated by calculating the correlation coefficient given by

$$
I=\frac{n \sum T_{i} V_{i}-\sum T_{i} \sum V_{i}}{\left[n \sum T_{i}^{2}-\left(\sum T_{i}\right)^{2}\right]^{\frac{1}{2}}\left[n \sum V_{i}^{2}-\left(\sum V_{i}\right)^{2}\right]^{\frac{1}{2}}}
$$

Solution of these equations yields the torque/voltage relationship

$$
V[\mathrm{mV}]=.4222+12.19 T
$$

with

$$
r=0.9999
$$

for positive torque and

$$
V[\mathrm{mV}]-0.0000+12.19 \mathrm{~T}
$$


Table C1. Calibration data for the torque cell.

\begin{tabular}{|c|c|}
\hline $\begin{array}{c}\text { Torque } \\
{[\mathrm{Nm}]}\end{array}$ & $\begin{array}{c}\text { Voltage } \\
{[\mathrm{mV}]}\end{array}$ \\
\hline-40.0 & -483.0 \\
\hline-35.0 & -426.0 \\
\hline-30.0 & -361.0 \\
\hline-25.0 & -302.0 \\
\hline-20.0 & -240.0 \\
\hline-15.0 & -183.0 \\
\hline-10.0 & -120.0 \\
\hline-5.0 & -63.0 \\
\hline 0.0 & 0.0 \\
\hline 5.0 & 63.0 \\
\hline 10.0 & 121.0 \\
\hline 15.0 & 184.0 \\
\hline 20.0 & 243.0 \\
\hline 25.0 & 306.0 \\
\hline 30.0 & 365.0 \\
\hline 35.0 & 428.0 \\
\hline 40.0 & 488.0 \\
\hline & \\
\hline
\end{tabular}


with

$$
r=0.9999
$$

for negative torques. The result is a transducer gain of $82 \mathrm{Nm} /$ Volt. The calibration curve is shown in Figure $\mathrm{C} 1$ along with the data points used to find the torque/voltage relationship. 


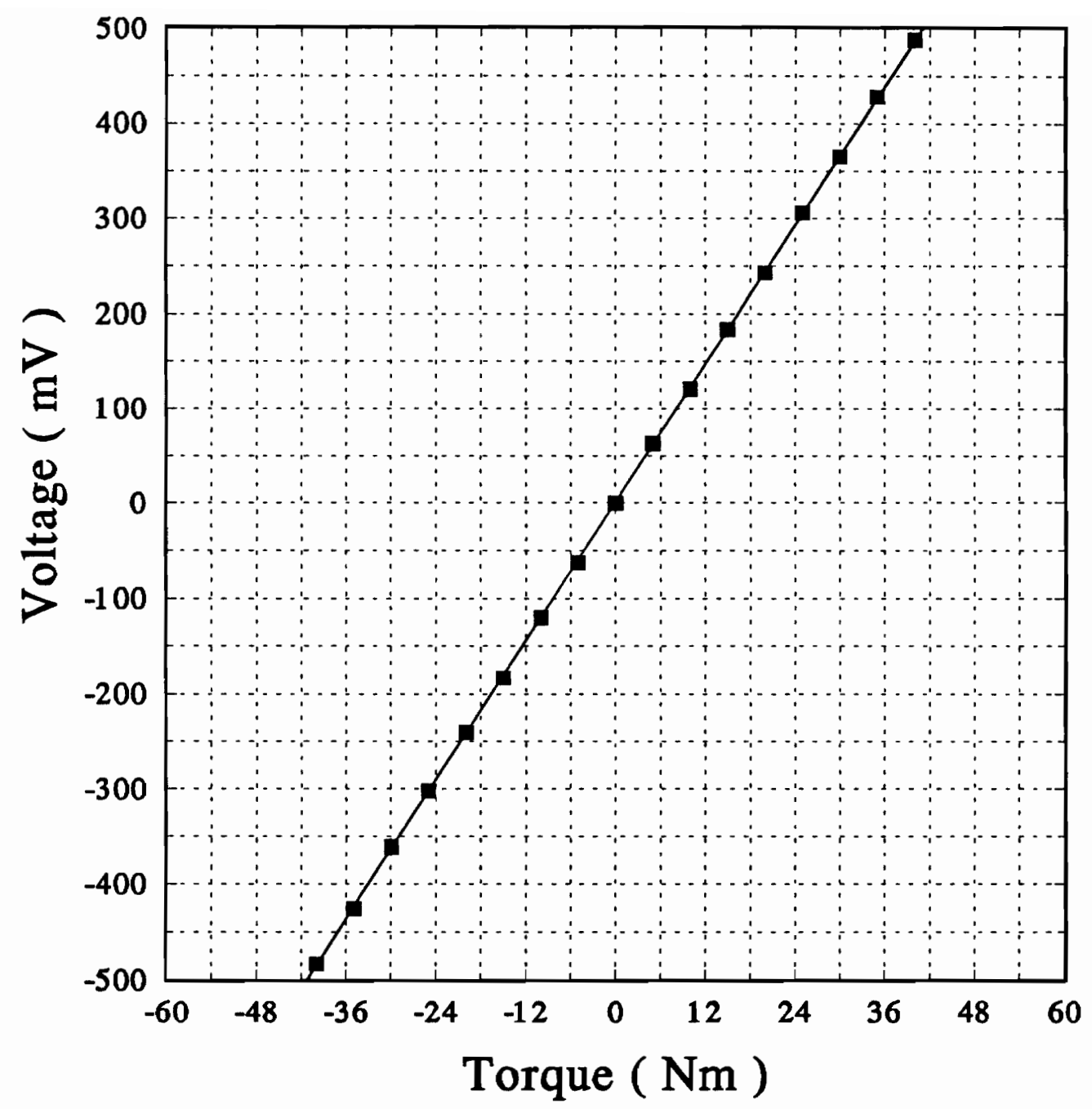

Figure $\mathrm{C} 1-$ Calibration Curve for the Torque Cell. 


\section{Appendix D. Calibration of Accelerometer}

The original calibration of the accelerometer is included in Figure D1 below. The gain for this transducer is $781.4 \mathrm{~m} / \mathrm{s}^{2} /$ volt.

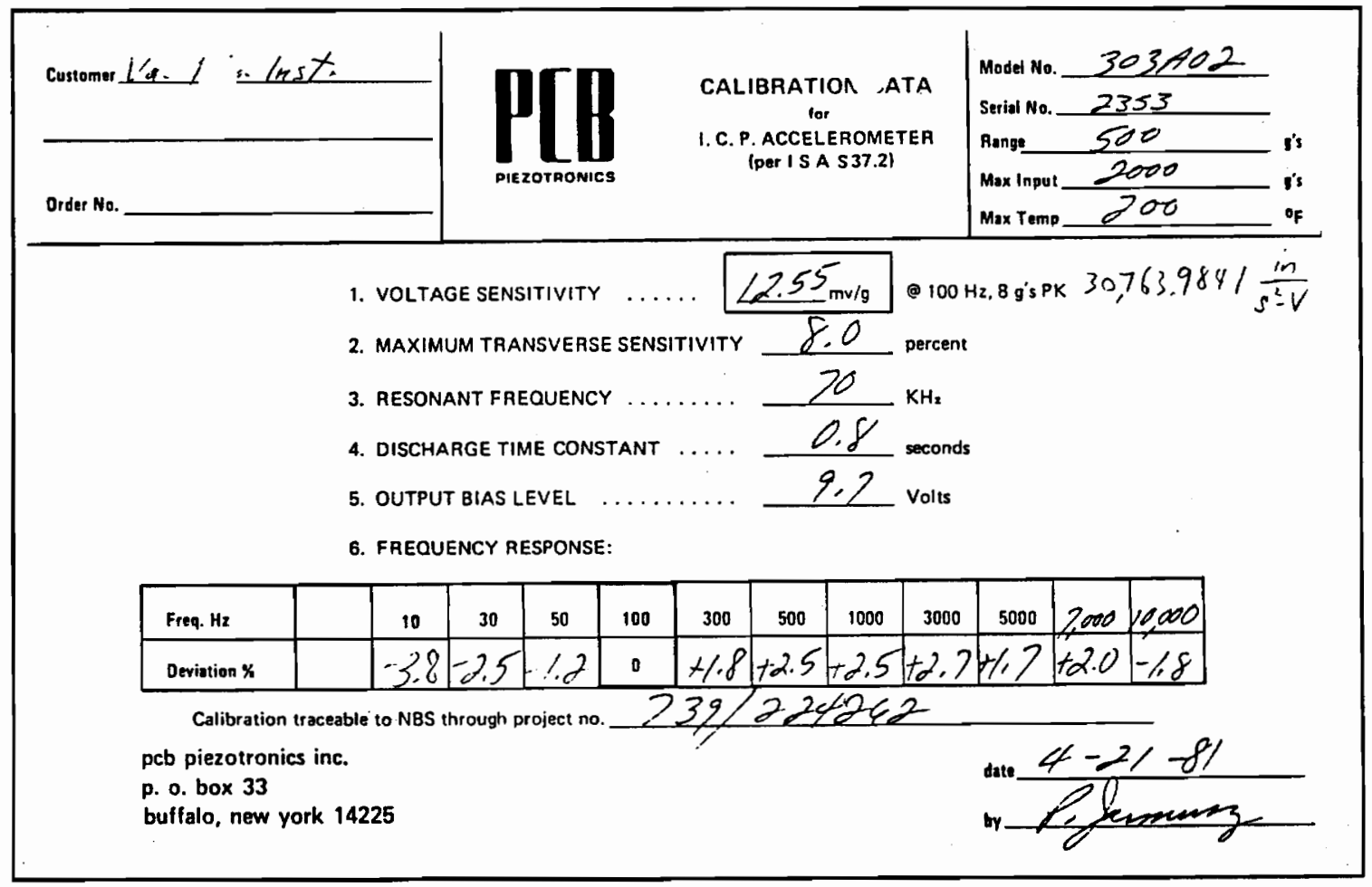

Figure D1 - Calibration and specification of the accelerometer. 


\section{Appendix E. Calibration of the Microphone}

The $1 / 2$ inch microphone was calibrated with a B \& K sound level calibrator. The calibrator produced a $1,000 \mathrm{~Hz}$ tone at $93.8 \mathrm{~dB}$. Three samples were taken and the output voltage from the transducer was digitally acquired using the Data Translation data acquisition board. The amplitude for each test was \pm 0.06 volts. To find the relationship between the sound pressure and voltage a conversion from sound power level, $L_{P}$, to $\mathrm{Pa}$ was required.

$$
\begin{aligned}
L_{P} & =10 \log \left[\left(\frac{p_{r m s}}{p_{\text {ref }}}\right)^{2}\right]-20 \log \left[\frac{p_{\text {rms }}}{p_{\text {ref }}}\right] \\
\text { where } p_{\text {ref }}-20(10)^{-6}[\mathrm{~Pa}] & \\
p_{\text {rms }} & =2(10)^{\left[L_{p}-100\right] / 20}=2(10)^{[93.8-100] / 20} \\
& =0.979755
\end{aligned}
$$

Note the amplitude of the pressure wave is equal to 1.414 times the root mean square pressure. Therefore

$$
P=\sqrt{2} p_{r m s}-1.38[P a]
$$

This pressure corresponds to the 0.06 volt amplitude. Therefore the microphone gain is $23.088 \mathrm{~Pa} / \mathrm{V}$. 


\section{Appendix F. Listing of Lumped Parameter Model}

100 DIM T1(2000), T1D(2000), T1DD (2000)

110 DIM T2(2000), T2D (2000), T2DD (2000)

120 DIM X2 (4), Y2 (4), F2 (4), X1 (4), Y1 (4), F1 (4)

130 OPEN "c:filename.dat"FOR OUTPUT AS \#1

Define the integration time step

$140 \mathrm{H}=.00002$

$150 \mathrm{HH}=.00001$

160 TIME $=-.00002$

Define system parameters and the mean and critical torque

$170 \mathrm{KE}=665$

$180 \mathrm{~KB}=3745$

190 INPUT"Enter the damping coefficient ";CE

$200 \mathrm{JC}=1 / 1200: \mathrm{JE}=1 / 65000$ !

$210 \mathrm{TK}=8$

220 TCRIT $=10.7$

Define the input velocity and the initial conditions, ie., the displacement and velocity of each mass

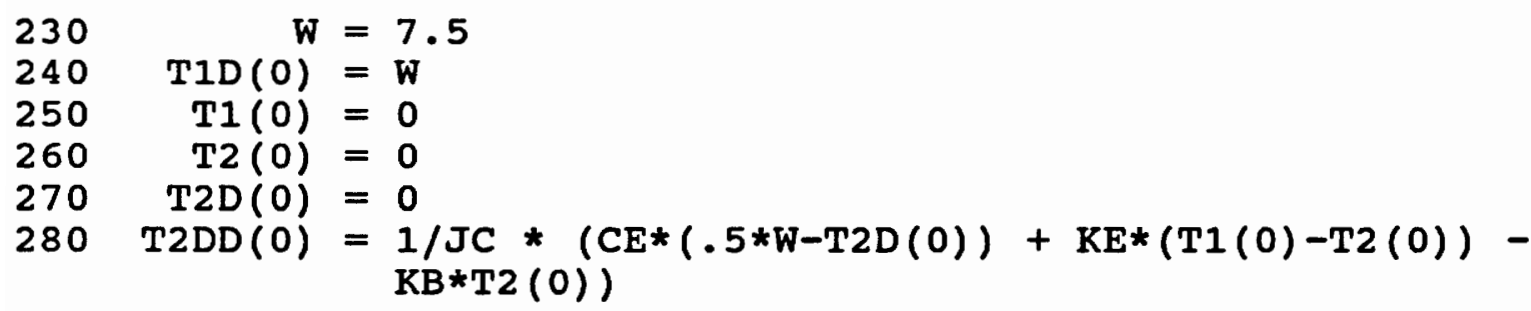

This loop solves the equations of motion during the stick phase of the cycle using a fixed time step Runge-Kutta numerical solution

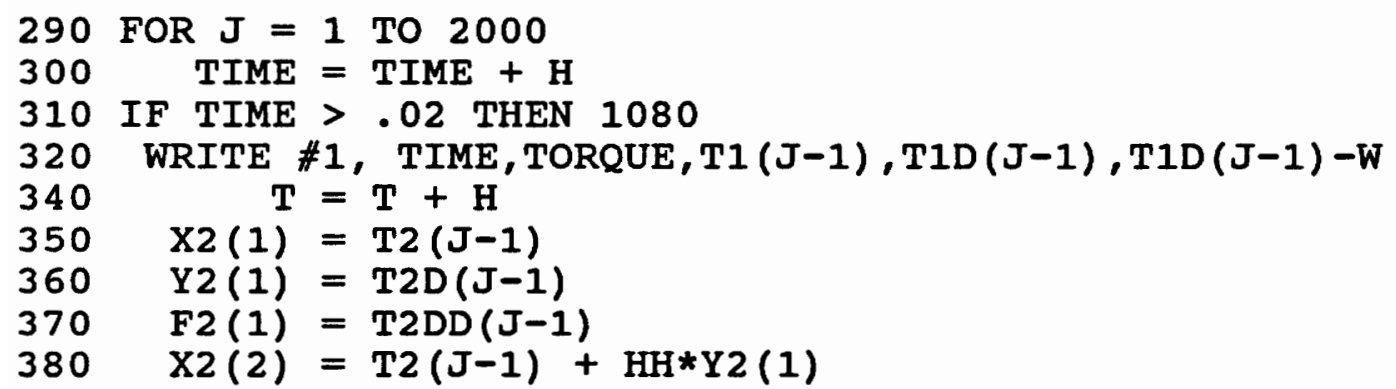




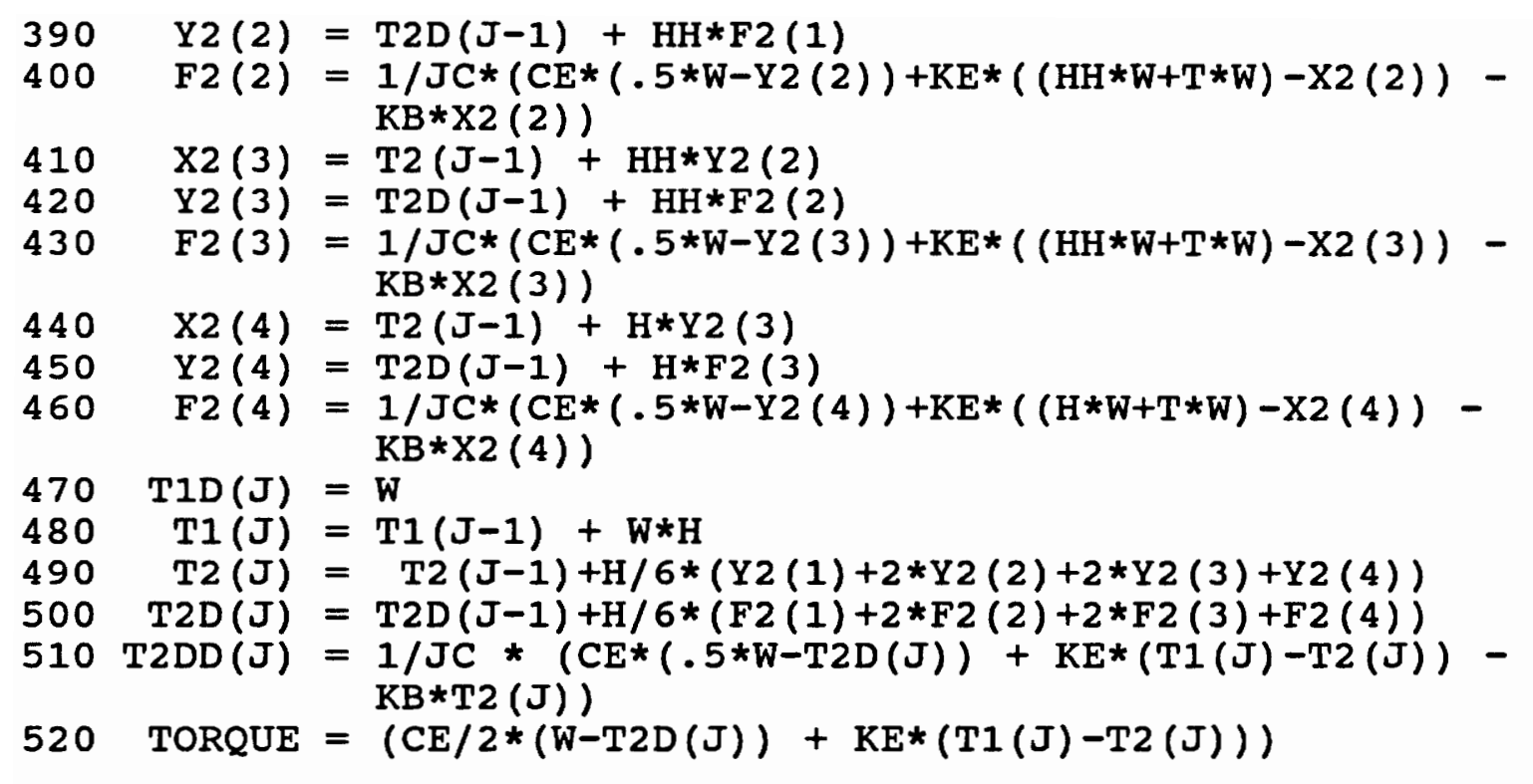

When the critical torque is reached, the stick phase ends.

540 IF TORQUE > TCRIT THEN 560

550 NEXT J

The final values of displacement and velocity become the initial conditions to solve the equations of motion during the slip phase.

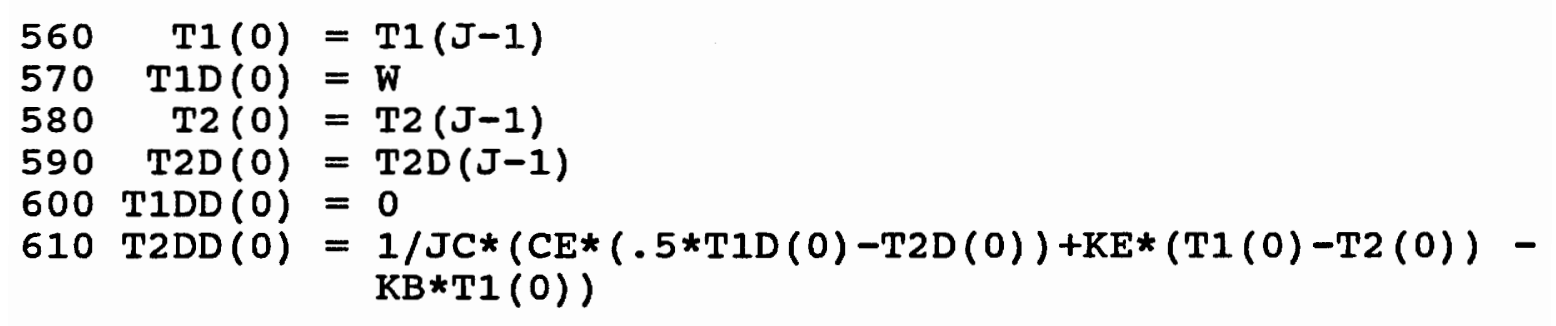

Again a fixed time step Runge-Kutta numerical solution is used to solve the equations of motion in the following loop

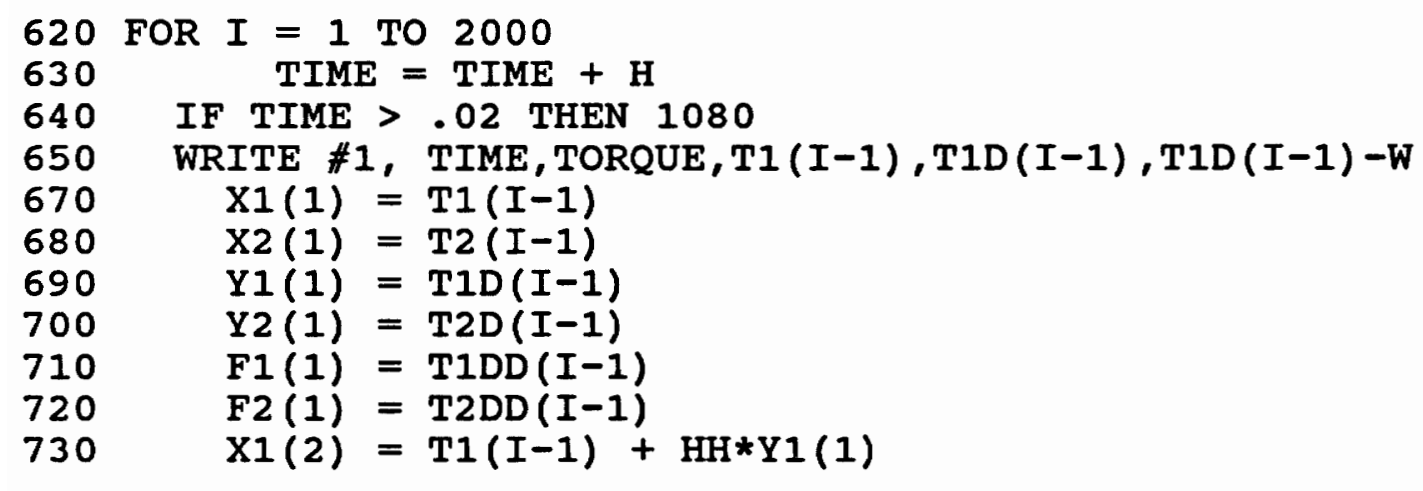




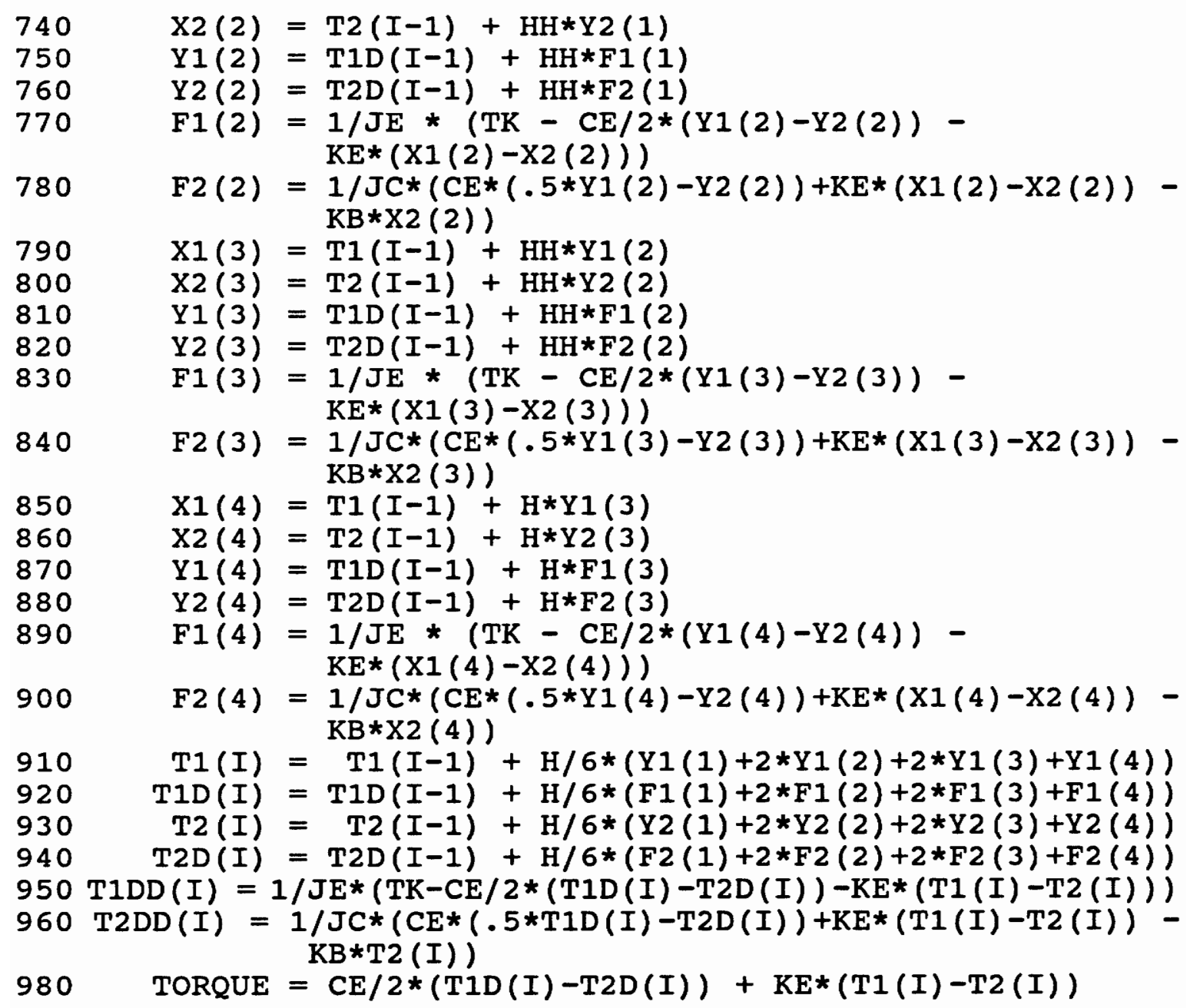

The slip phase continues until the relative velocity of the shaft and elastomer is zero

990 IF T1D(I) > W THEN 1010

1000 NEXT I

The final values of displacement and velocity become the initial conditions to solve the equations of motion during the stick phase.

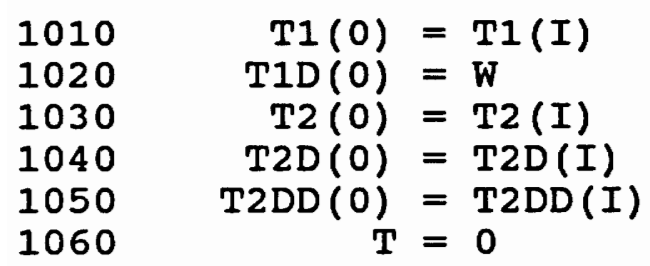

Resume the stick solution 
1070 GOTO 290

1080 CLOSE 1

1090 END 


\section{Appendix G. Surface Temperature Model}

A one-dimensional heat conduction model was developed [28] to ensure frictional heating does not affect the dynamic modulus of the elastomer. If the temperature rose sufficiently, the friction torque/displacement relationship would become nonlinear. The surface temperature rise and the temperature distribution throughout the bulk is determined analytically. There are two inputs to the system. These are the angular velocity of the shaft, $\ell_{\text {shaff }}$, and the friction torque, $T$. The duration of energy input, $t$, is also required. Since the radial heat transfer solution is cumbersome, a plane wall, semiinfinite solid model was used. One dimension heat conduction was chosen because the model will yield the most conservative estimate, ie., the largest temperature rise.

Plane heat source between two infinite bodies-Figure G1 is the schematic diagram showing the constant heat flux and conduction paths in each material. The friction generated heat flux is defined as

$$
q_{S}^{\prime \prime}\left[\frac{W}{m^{2}}\right]-\frac{T[N m] \omega\left[\frac{\mathrm{rad}}{\mathrm{s}}\right]}{A_{\text {contact }}\left[\mathrm{m}^{2}\right]}
$$

Since the all of the frictional heat must conduct through the materials

$$
q_{s}^{\prime \prime}-q_{A}^{\prime \prime}+q_{B}^{\prime \prime}
$$




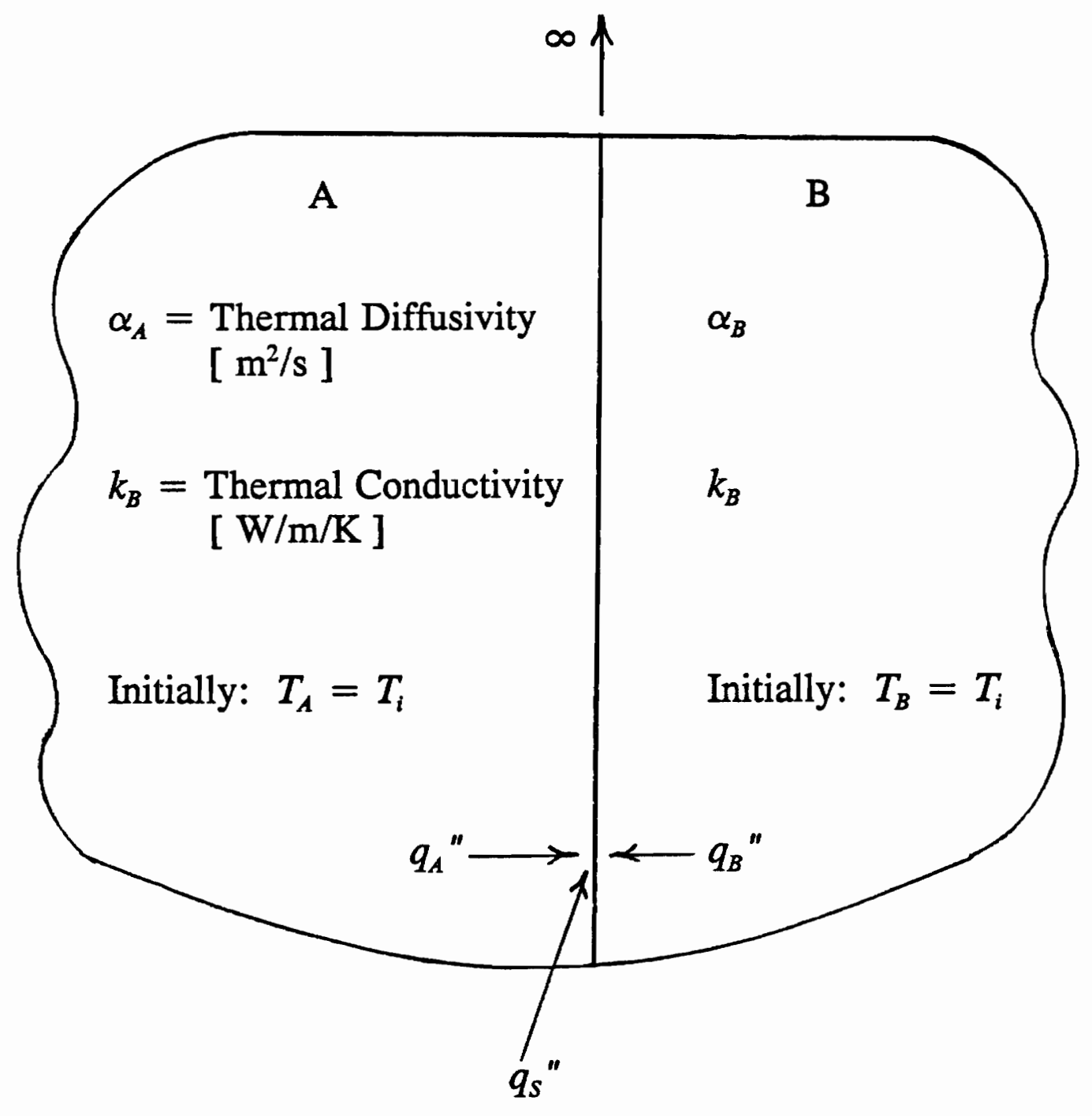

Figure G1 - Constant surface heat flux between two semiinfinite solids. 
$q^{\prime \prime}{ }_{A}$ is the fraction of friction heat conducted by the steel and $q^{\prime \prime}{ }_{B}$ is the fraction of heat conducted by the elastomer. $T_{i}$ is the initial temperature of the system and is equal to the ambient room temperature which was $23^{\circ} \mathrm{C}$.

Solution for the single region problem - The temperature model of the semiinfinite body where $q^{\prime \prime}{ }_{s}$ is known is shown in Figure G2(a). The temperature rise a distance $x$ from the heat flux source (the elastomer/shaft interface) is found using the thermal properties of the material. These are the thermal diffusivity, $\alpha\left[\mathrm{m}^{2} / \mathrm{s}\right]$, and the thermal conductivity, $k[\mathrm{~W} / \mathrm{m} / \mathrm{K}]$. The solution of the temperature distribution as a function of $x$ and $t$ is [29]

$$
T-T_{i}-2 \frac{q_{s}^{\prime \prime}}{k}\left[\frac{\alpha t}{\pi}\right] \exp \left[\frac{-x^{2}}{4 \alpha t}\right]-q_{S}^{\prime \prime} \frac{x}{k} \operatorname{erfc}\left[\frac{x}{2 \sqrt{\alpha t}}\right]
$$

The temperature distribution $T(x, t)$ will have the general trend in Figure G2(b).

Solution of the coupled problem-The surface temperatures, $T_{A}$ and $T_{B}$, are equal at $x=0$. Solving equation (G3) using the material properties and equating yields

$$
\begin{aligned}
2 \frac{q_{A}^{\prime \prime}}{k_{A}}\left[\frac{\alpha_{A} t}{\pi}\right]^{\frac{1}{2}} & =2 \frac{q_{B}^{\prime \prime}}{k_{B}}\left[\frac{\alpha_{B} t}{\pi}\right]^{\frac{1}{2}} \\
q_{A}^{\prime \prime} \frac{\alpha_{A}^{\frac{1}{2}}}{k_{A}} & =q_{B}^{\prime \prime} \frac{\alpha_{B}^{\frac{1}{2}}}{k_{B}} \\
& =\left[q_{S}^{\prime \prime}-q_{A}^{\prime \prime}\right] \frac{\alpha_{B}^{\frac{1}{2}}}{k_{B}}
\end{aligned}
$$




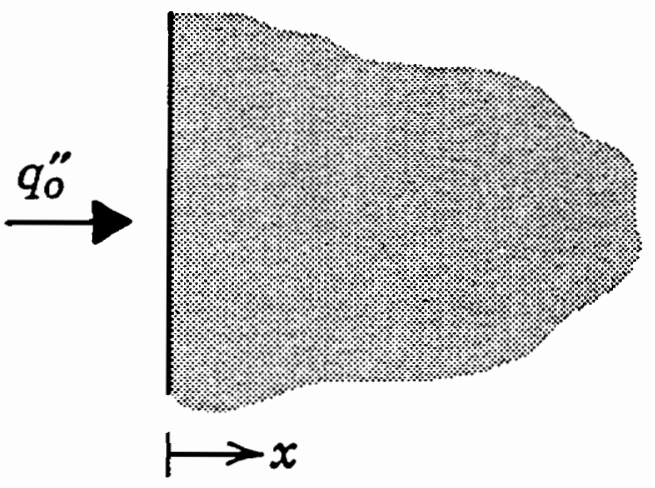

(a)

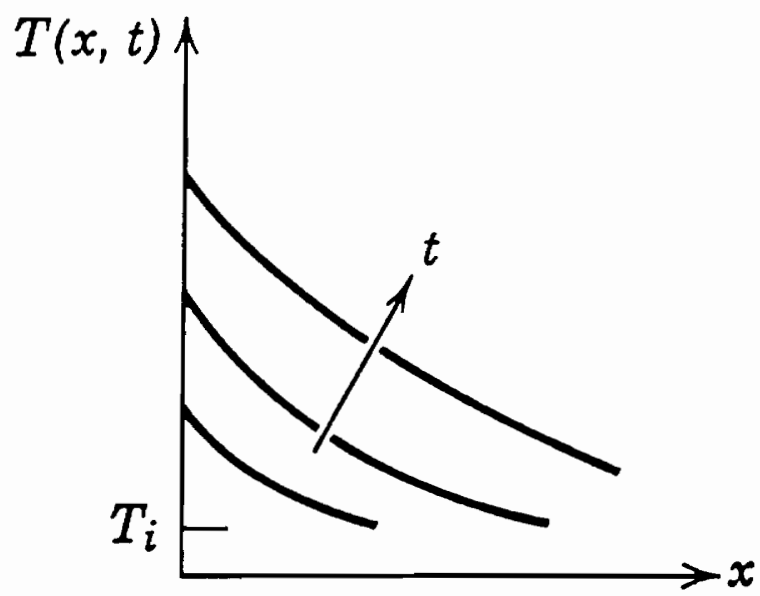

(b)

Figure G2 - (a) Semiinfinite solid with constant surface heat flux and (b) transient temperature distribution. 
The ratio of the heat conduction into each material is found to be

$$
\begin{aligned}
& \frac{q_{A}^{\prime \prime}}{q_{S}^{\prime \prime}}-\left[\frac{\frac{\alpha_{B}^{\frac{1}{2}}}{k_{B}}}{\left.\frac{\alpha_{A}^{\frac{1}{2}}}{k_{A}}+\frac{\alpha_{B}^{\frac{1}{2}}}{k_{B}}\right]}\right. \\
& \frac{q_{B}^{\prime \prime}}{q_{S}^{\prime \prime}}=1-\frac{q_{A}^{\prime \prime}}{q_{S}^{\prime \prime}}-\left[\frac{\frac{\alpha_{A}^{\frac{1}{2}}}{k_{B}}}{\frac{\alpha_{A}^{\frac{1}{2}}}{k_{A}}+\frac{\alpha_{B}^{\frac{1}{2}}}{k_{B}}}\right]
\end{aligned}
$$

These ratios, the distance, and the duration of heat source input are incorporated into equation (G3). The temperature distribution solutions in each material are

$$
\begin{aligned}
& T-T_{i}-2 \frac{q_{A}^{\prime \prime}}{k_{A}}\left[\frac{\alpha_{A} t}{\pi}\right]^{\frac{1}{2}} \exp \left[\frac{-x^{2}}{4 \alpha_{A} t}\right]-q_{A}^{\prime \prime} \frac{x}{k_{A}} \operatorname{erfc}\left[\frac{x}{2 \sqrt{\alpha_{A} t}}\right] \\
& T-T_{i}=2 \frac{q_{B}^{\prime \prime}}{k_{B}}\left[\frac{\alpha_{B} t}{\pi}\right]^{\frac{1}{2}} \exp \left[\frac{-x^{2}}{4 \alpha_{B} t}\right]-q_{A}^{\prime \prime} \frac{x}{k_{B}} \operatorname{erfc}\left[\frac{x}{2 \sqrt{\alpha_{B} t}}\right]
\end{aligned}
$$

Thermal properties for each material are listed in Table G1.

Table G1. Thermal properties of 304 stainless steel and rubber.

\begin{tabular}{|c||c|c||}
\hline Material & $\begin{array}{c}\text { Diffusivity } \\
{\left[\mathrm{m}^{2} / \mathrm{s}\right]}\end{array}$ & $\begin{array}{c}\text { Conductivity } \\
{[\mathrm{W} / \mathrm{m} / \mathrm{K}]}\end{array}$ \\
\hline \hline Steel, A & $3.95(10)^{-6}$ & 14.9 \\
\hline Rubber, B & $6.69(10)^{-8}$ & 0.16 \\
\hline
\end{tabular}


Figure G3(a) is the analytical solution to the temperature distribution during a fluorocarbon experiment lasting one second with mean torque, $T=30 \mathrm{Nm}$, and angular velocity, $\omega_{\text {shaft }}=10 \mathrm{rad} / \mathrm{s}$. The one-dimensional semi-infinite solid assumption is valid since the boundary conditions are not violated. Each line represents a 0.05 second increment in time. Figure G3(b) is the temperature distribution at the actual experimental run time of 0.23 seconds. 


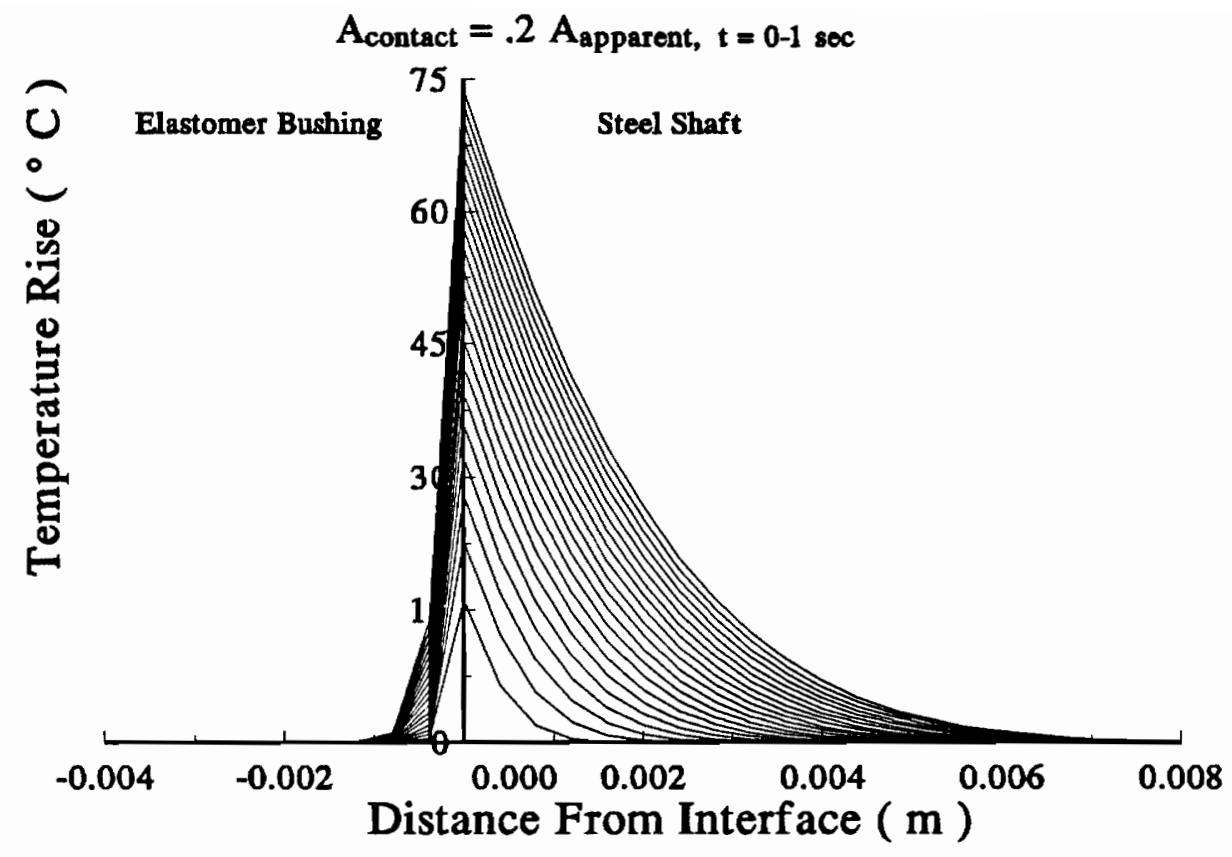

(a)

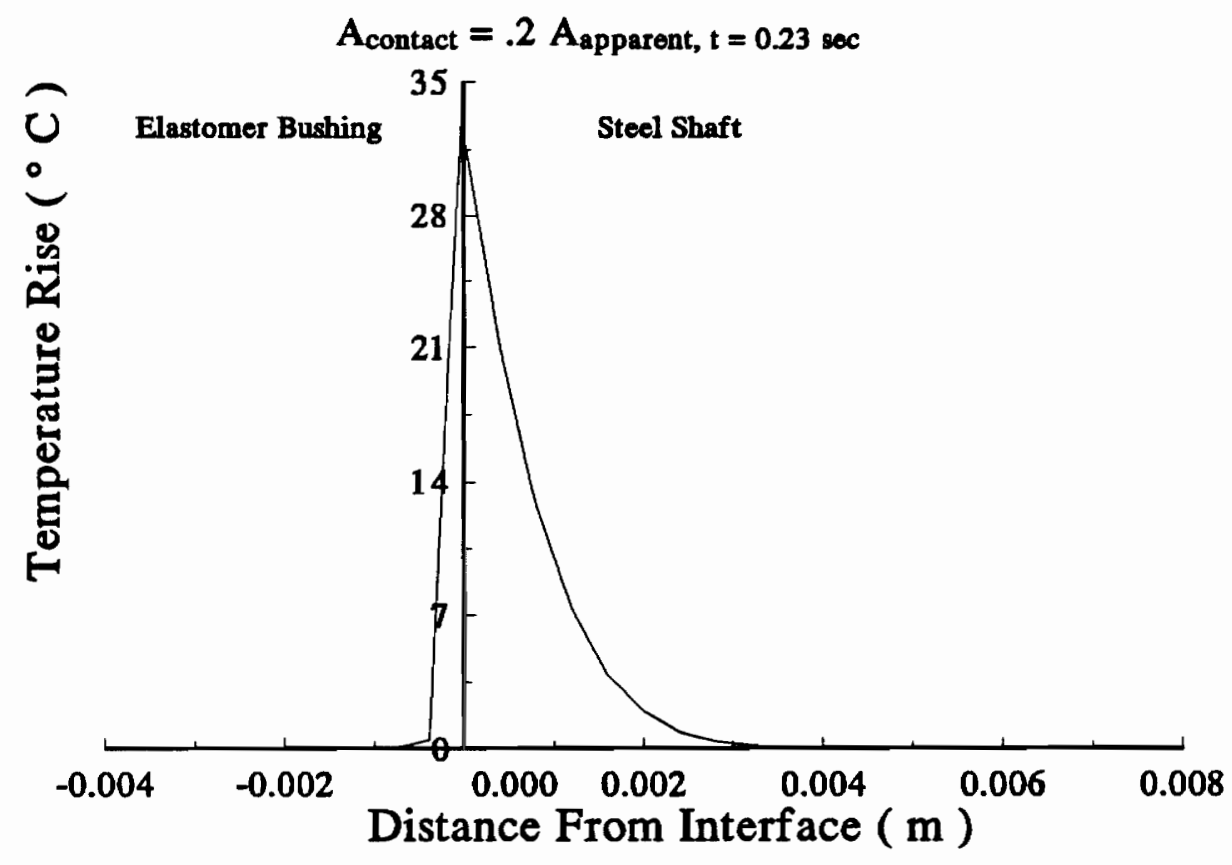

(b)

Figure G3 - Temperature distribution due to constant surface heat flux (a) temperature rise from 0.0 to $1.0 \mathrm{sec}$ and (b) temperature rise of $0.23 \mathrm{sec}$ experiment 


\section{Appendix H. Acoustic Response of the Apparatus}

The frequency response function is defined as the Fourier transform of the unit impulse response function [30]. It provides a measure of the single input/single output relationship of a system. The acoustic response of the apparatus was determined by experimentally by measuring the excitation of the system with a modal hammer and the sound pressure that radiated from the apparatus. Bendat and Piersol use the following method to determine the frequency response function.

The function is simply the cross spectrum estimate

$$
\hat{G}_{X Y}\left(f_{k}\right)=\frac{\Delta t}{N n_{\text {ave }}} \sum_{k-1}^{N} X\left(f_{k}\right)^{*} Y\left(f_{k}\right)
$$

divided by the auto spectrum estimate of the input.

$$
\hat{G}_{X X}\left(f_{k}\right)-\frac{\Delta t}{N n_{a v e}} \sum_{k=1}^{N} X\left(f_{k}\right) X^{*}\left(f_{k}\right)
$$

where $\mathrm{X}$ and $\mathrm{Y}$ are the fast Fourier transforms of the excitation and response. The asterisk is used to denote the complex conjugate. $N$ is the data set size, $\Delta t$ is the period, $n_{\text {ave }}$ is the number of data sets averaged, and $f_{k}$ is the discrete frequency. Computation of the frequency response functions in Figure $\mathrm{H} 1$ for each stiffness configuration of the apparatus is accomplished using equation (H3).

$$
\hat{H}\left(f_{k}\right)-\frac{\hat{G}_{X Y}\left(f_{k}\right)}{\hat{G}_{X X}\left(f_{k}\right)}
$$




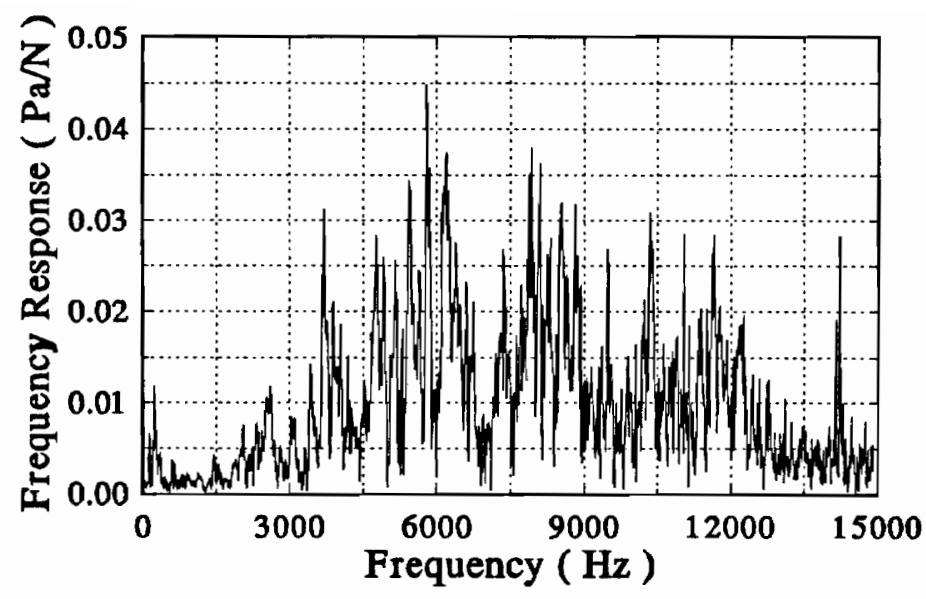

(a)

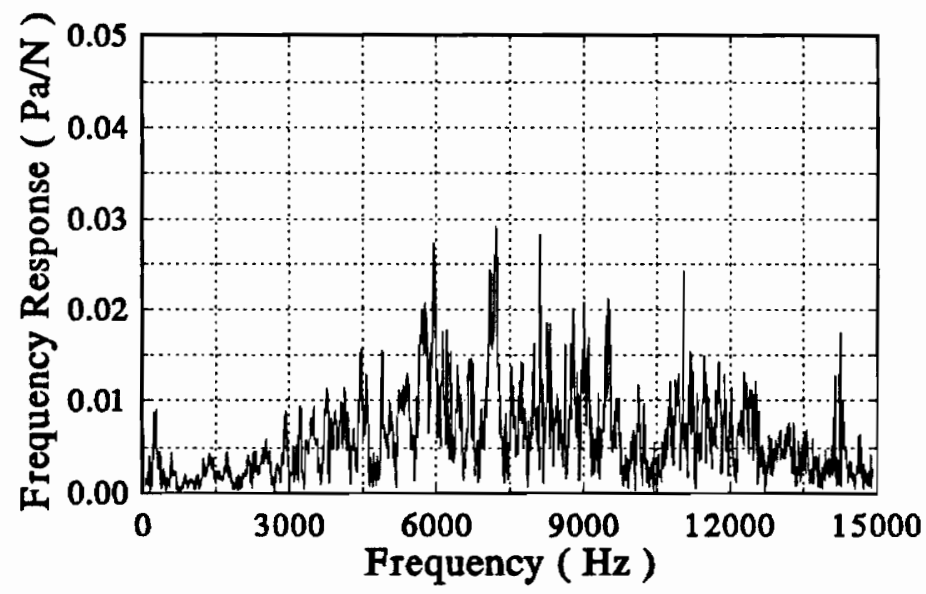

(b)

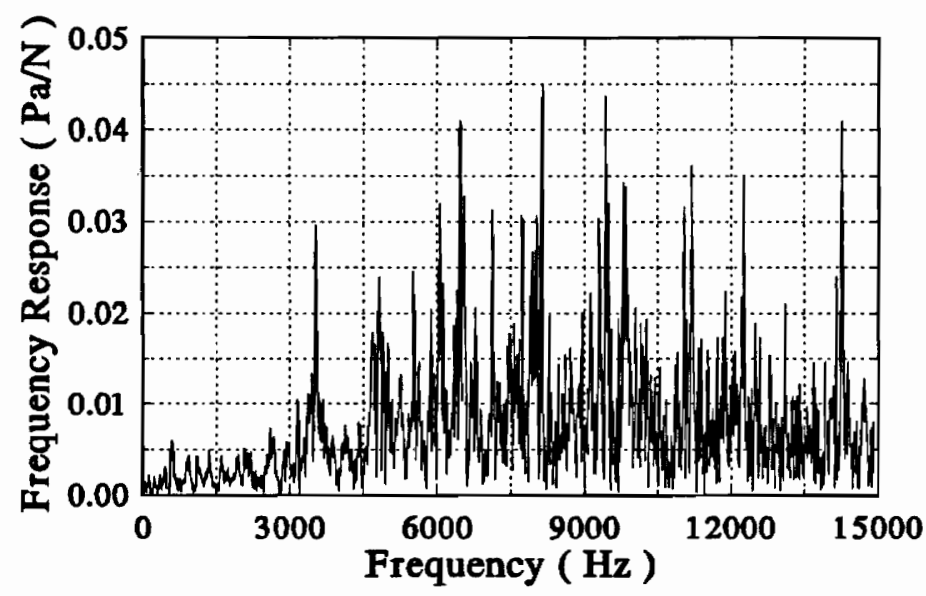

(c)

Figure $\mathrm{H} 1$ - Acoustic frequency response function for (a) the $12.3 \mathrm{~cm}$ beam length condition, (b) the $9.2 \mathrm{~cm}$ beam length condtion, and (c) the fixed clamp condition. 


\section{Vita}

The author was born on 1 February 1966 in Dover, New Jersey to Gino and Dorothy De Togni. The youngest of four children, he was raised in Succasunna, New Jersey. At eighteen years of age he began his higher education at Virginia Polytechnic Institute and State University. While completing the requirements for his undergraduate degree, he participated in two summer internships at ARDEC, Picatinney Arsenal in New Jersey. He received his Bachelor of Science, Mechanical Engineering Degree in May of 1989 with aspirations of attending graduate school. In August 1989 he commenced work towards his Master of Science degree. Upon completion of this he will pursue his career working in applied research and product development.

\section{Robert \& De Fogni}

